Supporting Information

for

\title{
Multistage Reversible Tg Photo-Modulation and Hardening of Hydrazone-Containing Polymers
}

Sirun Yang, ${ }^{1}$ Jared D. Harris, ${ }^{1}$ Aloshious Lambai, ${ }^{2}$ Laura L. Jeliazkov, ${ }^{1}$ Gaurav Mohanty, ${ }^{2}$ Hao Zeng, ${ }^{2}$ Arri Priimagi, ${ }^{2}$ and Ivan Aprahamian ${ }^{1 *}$

1. Department of Chemistry, Dartmouth College, Hanover, New Hampshire 03755, United States

2. Faculty of Engineering and Natural Sciences, Tampere University, Korkeakoulunkatu 10, Tampere, 33720 Finland

Email:ivan.aprahamian@dartmouth.edu 
Table of Contents

1. General Methods $\quad 3$

2. Synthesis $\quad 5$

3. NMR Spectroscopy Characterization $\quad 22$

4. Switching Behavior of the Monomers $\quad 31$

5. Switching Behavior of the Polymers 36

6. Photostationary States of the Polymers in solution 41

7. Photostationary States of the Polymers in thin film state 46

8. Photoisomerization Quantum Yield of Polymers $\quad 50$

$\begin{array}{lr}\text { 9. Kinetic Studies } & 67\end{array}$

10. Summary of Glass Transition Temperature Measurements for the Hydrazone$\begin{array}{ll}\text { Containing Polymers } & 68\end{array}$

11. Nanoindentation for photo-stiffening verification $\quad 72$

12. Locking of Glass Transition Temperature through Photoisomerization 78

$\begin{array}{ll}\text { 13. References } & 81\end{array}$ 


\section{General Methods}

Materials and Methods:

All reagents and starting materials were purchased from commercial vendors and used as supplied unless otherwise indicated. All experiments were conducted under air unless otherwise noted. Compounds were purified by column chromatography using silica gel (SiliCycle ${ }^{\circledR}, 60 \AA$, 230-400 mesh) as stationary phase and solvent mixtures used during chromatography were reported as volume ratios unless otherwise noted. Deuterated solvents were purchased from Cambridge Isotope Laboratories, Inc. and used as received. ${ }^{1} \mathrm{H}$ NMR, ${ }^{13} \mathrm{C}$ NMR and 2D NMR spectra were recorded on a 500 or $600 \mathrm{MHz}$ NMR spectrometer, with working frequencies of 500.13 or $600.13 \mathrm{MHz}$ for ${ }^{1} \mathrm{H}$ nuclei, and 125.8 or $150.9 \mathrm{MHz}$ for ${ }^{13} \mathrm{C}$ nuclei, respectively. Chemical shifts are quoted in ppm relative to tetramethylsilane (TMS), using the residual solvent peak as the reference standard. ESI mass spectra were obtained on a Waters Quattro II ESI mass spectrometer. Melting points were measured on an Electrothermal Thermo Scientific IA9100X1 digital melting point instrument. UV-Vis spectra were recorded on a Shimadzu UV-1800 UV-Vis spectrophotometer. GPC was performed at $50{ }^{\circ} \mathrm{C}$ using a Waters e2695 using THF and Styragel ${ }^{\circledR}$ HR 2/4/5 THF as the mobile and stationary phases with 2414 RI detector, respectively.

Irradiation experiments were conducted with a stand-alone xenon arc lamp system (Model: LB-LS/30, Sutter Instrument Co.), outfitted with a SMART SHUTTER controller (Model: LB10B/IQ, Sutter Instrument Co.) and a liquid light guide LLG/250. 340 (part number: 340HC10-25) $\left(\mathrm{I}_{0}=1.52 \mathrm{E}-07\right.$ quanta $\cdot \mathrm{s}^{-1}$ ), 365 (part number: $365 \mathrm{HC} 10-25$ ), 375 (part number: $375 \mathrm{HC} 10-25$ ), 410 (part number: 410FS10-25) $\left(\mathrm{I}_{0}=1.53 \mathrm{E}-07\right.$ quanta $\left.\cdot \mathrm{s}^{-1}\right), 442$ (part number: 442F5X10-25), and 480 (part number: $480 \mathrm{HC} 10-25) \mathrm{nm}$ light filters, purchased from Andover Corporation, were used in the irradiation experiments. For UV measurement, irradiation time of $410 \mathrm{~nm}$ is around $100-500$ $\mathrm{s}$ to reach the photostationary state depending on the photoisomerization efficiency. In the case of $340 \mathrm{~nm}$, irradiation time is around $30 \mathrm{~s}$.

\section{Differential Calorimetry Measurements:}

Differential Calorimetry (DSC) experiments were carried out using a TA Instruments differential scanning calorimeter using a heat/cool/heat program cycling from 35 to 150 to 0 to $150{ }^{\circ} \mathrm{C}$ at a scan rate of $10{ }^{\circ} \mathrm{C} \mathrm{min}^{-1}$. Pristine precipitates (>99\% $\mathrm{Z}$ by ${ }^{1} \mathrm{H}$ NMR spectroscopy) or $E$ enriched (via irradiation to photostaionary state (PSS)) polymers ( 6-10 mg) were loaded into aluminum 
Tzero pans for the measurements. The pristine samples were simply weighed into the pans and analyzed as powders. $E$ enriched samples were prepared by dissolving ca. $30 \mathrm{mg}$ of pristine sample in ca. $1.5 \mathrm{~mL}$ dichloromethane $\left(\mathrm{CD}_{2} \mathrm{Cl}_{2}\right)$ and irradiating the solution with $410 \mathrm{~nm}$ light. ${ }^{1} \mathrm{H} \mathrm{NMR}$ spectroscopy was used to monitor the isomerization process until the samples reached $\mathrm{PSS}_{410}$, upon which the solvent was evaporated by blowing compressed air over the solution, followed by high vacuum drying overnight. The resulting flakey solids were then directly weighed in DSC pans and analyzed as described above. 


\section{Synthesis}

\subsection{Synthesis of Hydrazone-Based Methacrylate Monomers}

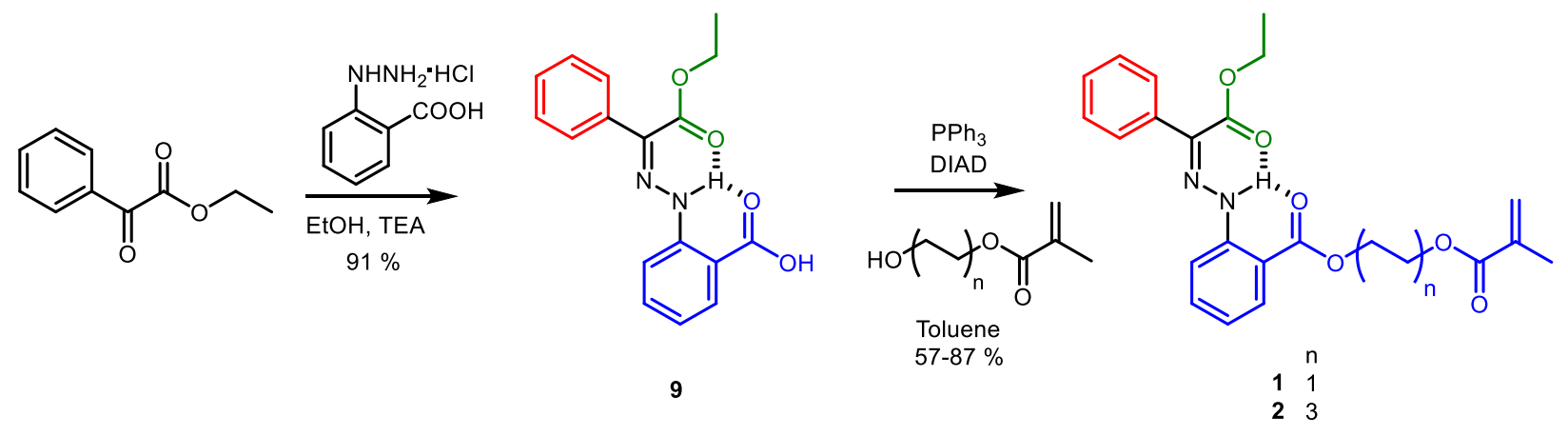

Scheme S1. Summary of the synthetic pathway used to access monomers 1 and 2.<smiles>CCOC(=O)C1=NN2c3ccccc3C(=O)O[C@H]2N=C1c1ccccc1</smiles>

9: This compound was synthesized using a reported procedure ${ }^{\mathrm{S} 1}$ and its identity confirmed by comparing its ${ }^{1} \mathrm{H}$ NMR spectrum with the one in the literature. ${ }^{1} \mathrm{H}$ NMR $\left(600 \mathrm{MHz}\right.$, DMSO- $\left.d_{6}\right) \delta$ 13.41 (s, 1H), 13.34 (s, 1H), 7.93 (dd, 1H, $J=1.5,8.1 \mathrm{~Hz}), 7.69$ (m, 2H), 7.59 (m, 1H) $7.42(\mathrm{~m}$, 2H), $7.37(\mathrm{~m}, 1 \mathrm{H}), 7.01(\mathrm{~m}, 1 \mathrm{H}), 4.40(\mathrm{q}, 2 \mathrm{H}, J=7.2 \mathrm{~Hz}), 1.33(\mathrm{t}, 3 \mathrm{H}, J=6.9 \mathrm{~Hz}) \mathrm{ppm}$.<smiles></smiles> 
1: Hydroxyethyl methacrylate (0.982 g, $7.55 \mathrm{mmol}, 1.50$ eq.), 9 (1.575 g, 5.04 mmol, 1.00 eq.), and triphenylphosphine ( $1.576 \mathrm{~g}, 6.01 \mathrm{mmol}, 1.19 \mathrm{eq}$.) were added to a dry $250 \mathrm{~mL}$ round bottomed Schlenk flask under a $\mathrm{N}_{2}$ atmosphere. The flask was sealed and purged of $\mathrm{O}_{2}$ with $\mathrm{N}_{2}$ for 15 minutes before adding deoxygenated anhydrous toluene $(50 \mathrm{~mL})$ via a syringe. The resulting mixture was stirred for 5 minutes before cooling in a $0{ }^{\circ} \mathrm{C}$ ice bath. Diisopropyl azodicarboxylate $(1.30 \mathrm{~mL}$, $6.60 \mathrm{mmol}, 1.31$ eq.) was then added to the mixture dropwise. The solution was allowed to warm up to room temperature and stirred for $4 \mathrm{~h}$ and then filtered through Celite and washed with ethyl acetate. After removal of the solvent under reduced pressure, the product was purified by column chromatography (4:1 hexanes: ethyl acetate) affording 1 as a yellow solid; yield 87 \%. m.p. 79.8 $-80.5{ }^{\circ} \mathrm{C} .{ }^{1} \mathrm{H}$ NMR $\left(500 \mathrm{MHz}, \mathrm{CD}_{2} \mathrm{Cl}_{2}\right) \delta 13.75$ (s, 1H), 8.01 (dd, $\left.1 \mathrm{H}, J=1.0,8.0 \mathrm{~Hz}\right), 7.93$ (dt, $1 \mathrm{H}, J=0.6,8.5 \mathrm{~Hz}), 7.69(\mathrm{~m}, 2 \mathrm{H}), 7.53(\mathrm{~m}, 1 \mathrm{H}), 7.38(\mathrm{~m}, 3 \mathrm{H}), 6.98(\mathrm{~m}, 1 \mathrm{H}), 6.13$ (sextet, 1H, $J$ $=1.0 \mathrm{~Hz}), 5.59(\mathrm{p}, 1 \mathrm{H}, J=1.5 \mathrm{~Hz}), 4.62(\mathrm{t}, 2 \mathrm{H}, J=4.8 \mathrm{~Hz}), 4.51(\mathrm{t}, 2 \mathrm{H}, J=4.8 \mathrm{~Hz}), 4.42(\mathrm{q}, 2 \mathrm{H}$, $J=7.2 \mathrm{~Hz}), 1.94(\mathrm{q}, 3 \mathrm{H}, J=0.8 \mathrm{~Hz}), 1.38(\mathrm{t}, 3 \mathrm{H}, J=7.3 \mathrm{~Hz}) \mathrm{ppm} .{ }^{13} \mathrm{C} \mathrm{NMR}\left(151 \mathrm{MHz}, \mathrm{CD}_{2} \mathrm{Cl}_{2}\right)$ $\delta 167.58,167.51,163.09,146.75,137.07,136.77,135.16,132.41,131.62,129.14,128.54,128.46$, 126.18, 121.00, 115.11, 112.86, 63.35, 62.91, 62.06, 18.58, 14.54 ppm. Hi-Res ESI-MS: $m / z$ found $\left[\mathrm{M}-\mathrm{H}^{+}\right]$for $\mathrm{C}_{23} \mathrm{H}_{25} \mathrm{~N}_{2} \mathrm{O}_{6}{ }^{+} 425.1713$ (calcd. 425.1713).

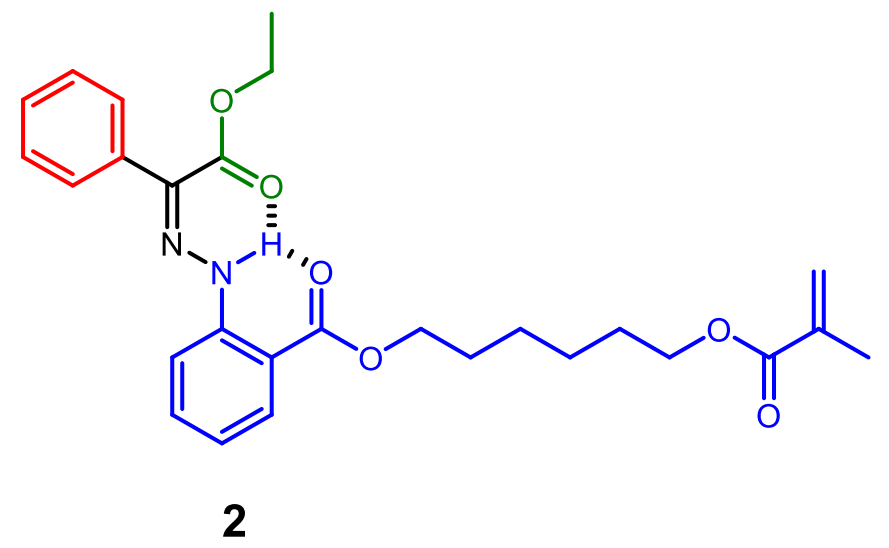

2: 6-Hydroxyhexyl methacrylate (1.170 g, 6.28 mmol, 1.27 eq.), 9 (1.562 g, 4.95 mmol, 1 eq.), and triphenylphosphine $(1.575 \mathrm{~g}, 6.00 \mathrm{mmol}, 1.21$ eq. $)$ were added to a dry $250 \mathrm{~mL}$ roundbottomed Schlenk flask under a $\mathrm{N}_{2}$ atmosphere. The flask was sealed and purged of $\mathrm{O}_{2}$ with $\mathrm{N}_{2}$ for 15 minutes. Deoxygenated anhydrous toluene $(50 \mathrm{~mL})$ was added to the flask via a syringe. The mixture was stirred at room temperature for 5 minutes before cooling in a $0{ }^{\circ} \mathrm{C}$ ice bath. Diisopropyl azodicarboxylate $(1.28 \mathrm{~mL}, 6.50 \mathrm{mmol}, 1.31$ eq.) was then added to the mixture dropwise via a syringe. The stirred solution was allowed to come to room temperature over $4 \mathrm{~h}$ at 
which point the reaction mixture was filtered through Celite and washed with ethyl acetate. After the removal of solvent under reduced pressure, the product was purified by column chromatography (5:1 hexanes:ethyl acetate), yielding 2 as a yellow oil; yield $70 \% .{ }^{1} \mathrm{H}$ NMR (600 $\left.\mathrm{MHz}, \mathrm{CD}_{2} \mathrm{Cl}_{2}\right) \delta 13.79(\mathrm{~s}, 1 \mathrm{H}), 8.01(\mathrm{dd}, 1 \mathrm{H}, J=1.5,8.1 \mathrm{~Hz}), 7.93(\mathrm{~d}, 1 \mathrm{H}, J=8.4 \mathrm{~Hz}), 7.69(\mathrm{~m}$, 2H), 7.52 (m, 1H), 7.40 (m, 2H), 7.35 (m, 1H), 6.97 (m, 1H), 6.05 (m, 1H), 5.54 (p, 1H, $J=1.5$ Hz), 4.43 (q, 2H, $J=7.0 \mathrm{~Hz}), 4.37$ (t, 2H, $J=6.6 \mathrm{~Hz}), 4.14$ (t, 2H, $J=6.6 \mathrm{~Hz}), 1.92$ (q, 3H, $J=$ $1.2 \mathrm{~Hz}), 1.83$ (p, 2H, $J=6.9 \mathrm{~Hz}), 1.72(\mathrm{p}, 2 \mathrm{H}, J=7.1 \mathrm{~Hz}), 1.51(\mathrm{~m}, 4 \mathrm{H}), 1.38(\mathrm{t}, 3 \mathrm{H}, J=7.2 \mathrm{~Hz})$ ppm. ${ }^{13} \mathrm{C}$ NMR (151 MHz, $\left.\mathrm{CD}_{2} \mathrm{Cl}_{2}\right) \delta 167.83,167.83$ (Overlapped), 163.08, 146.66, 137.31, $137.15,134.84,132.20,131.49,129.13,128.48,128.45,125.29,120.94,115.05,113.45,65.61$, $65.13,62.00,29.17,29.13,26.36,26.33,18.64,14.55 \mathrm{ppm}$. Hi-Res ESI-MS: $m / z$ found $\left[\mathrm{M}-\mathrm{H}^{+}\right]$ for $\mathrm{C}_{2} 7 \mathrm{H}_{33} \mathrm{~N}_{2} \mathrm{O}_{6}{ }^{+} 481.2340$ (calcd. 481.2339).

\subsection{General Synthetic Procedure for the a-Keto Esters}

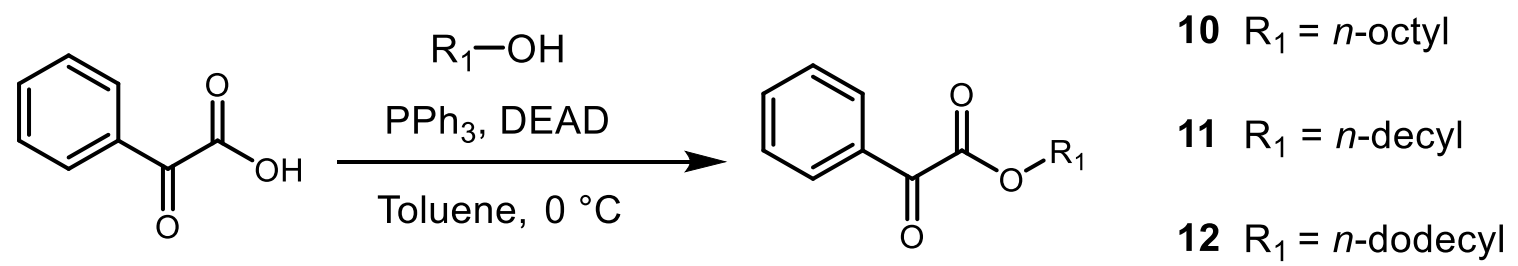

Scheme S2. General synthetic pathway for accessing the $\alpha$-keto esters.

The $\alpha$-keto esters were synthesized using a Mitsunobu reaction. DEAD $(6 \mathrm{mmol})$ was added dropwise to a solution of benzoylformic acid (1 equiv.), the appropriate alcohol ( $\left.\mathrm{R}_{1}-\mathrm{OH}\right)$ (1 equiv.) and $\mathrm{PPh}_{3}$ (1 equiv.) in toluene $(30 \mathrm{~mL})$ at $0{ }^{\circ} \mathrm{C}$. The reaction was left to warm up to room temperature and stirred for an additional 20 minutes. After removal of the solvent under reduced pressure, the product was purified by column chromatography affording the $\alpha$-keto esters as colorless oil.

10: The identity of this $\alpha$-keto ester was confirmed by comparing its ${ }^{1} \mathrm{H}$ NMR spectrum with the one in the literature. ${ }^{\mathrm{S} 2}{ }^{1} \mathrm{H} \mathrm{NMR}\left(500 \mathrm{MHz}, \mathrm{CDCl}_{3}\right) \delta 8.01(\mathrm{dt}, J=8.5,1.5 \mathrm{~Hz}, 2 \mathrm{H}), 7.72-7.62$ (m, 1H), $7.56-7.49(\mathrm{~m}, 2 \mathrm{H}), 4.39$ (t, $J=6.8 \mathrm{~Hz}, 2 \mathrm{H}), 1.82-1.73(\mathrm{~m}, 2 \mathrm{H}), 1.45-1.37(\mathrm{~m}, 2 \mathrm{H})$, $1.37-1.21$ (m, 8H), 0.88 (t, $J=7.0 \mathrm{~Hz}, 3 \mathrm{H}) \mathrm{ppm}$. 
11: This $\alpha$-keto ester was directly used in the next step after its purification using flash column chromatography. ${ }^{1} \mathrm{H}$ NMR $\left(500 \mathrm{MHz}, \mathrm{CDCl}_{3}\right) \delta 8.01(\mathrm{dt}, J=8.5,1.4 \mathrm{~Hz}, 2 \mathrm{H}), 7.69-7.64(\mathrm{~m}, 1 \mathrm{H})$, $7.56-7.48$ (m, 2H), 4.39 (t, $J=6.8 \mathrm{~Hz}, 2 \mathrm{H}), 1.83-1.72(\mathrm{~m}, 2 \mathrm{H}), 1.42-1.37(\mathrm{~m}, 2 \mathrm{H}), 1.37-1.21$ (m, 12H), $0.88(\mathrm{t}, J=7.0 \mathrm{~Hz}, 3 \mathrm{H}) \mathrm{ppm} ;{ }^{13} \mathrm{C}$ NMR $\left(151 \mathrm{MHz}, \mathrm{CDCl}_{3}\right) \delta 186.72,164.22,135.08$, $132.73,130.23,129.10,66.63,32.08,29.70,29.67,29.49,29.37,28.67,25.99,22.88,14.31 \mathrm{ppm}$. ESI-MS: $m / z$ found $\left[\mathrm{M}-\mathrm{H}^{+}\right]$for $\mathrm{C}_{18} \mathrm{H}_{27} \mathrm{O}_{3}{ }^{+} 291.10$ (calcd. 291.20).

12: This $\alpha$-keto ester was directly used in the next step after its purification using flash column chromatography. ${ }^{1} \mathrm{H}$ NMR (500 MHz, $\left.\mathrm{CDCl}_{3}\right) \delta 8.02-7.98(\mathrm{~m}, 2 \mathrm{H}), 7.69-7.63(\mathrm{~m}, 1 \mathrm{H}), 7.55-$ $7.49(\mathrm{~m}, 2 \mathrm{H}), 4.38(\mathrm{t}, J=6.8 \mathrm{~Hz}, 2 \mathrm{H}), 1.85-1.72(\mathrm{~m}, 2 \mathrm{H}), 1.41(\mathrm{dt}, J=15.0,6.6 \mathrm{~Hz}, 2 \mathrm{H}), 1.35-$ $1.23(\mathrm{~m}, 18 \mathrm{H}), 0.88(\mathrm{t}, J=7.0 \mathrm{~Hz}, 3 \mathrm{H}) \mathrm{ppm} ;{ }^{13} \mathrm{C} \mathrm{NMR}\left(151 \mathrm{MHz}, \mathrm{CDCl}_{3}\right) \delta 186.71,164.22$, 135.08, 132.73, 130.23, 129.10, 66.63, 32.12, 29.84, 29.82, 29.75, 29.68, 29.55, 29.37, 28.68, 26.00, 22.90, 14.33 ppm. ESI-MS: $m / z$ found $\left[\mathrm{M}-\mathrm{H}^{+}\right]$for $\mathrm{C}_{20} \mathrm{H}_{31} \mathrm{O}_{3}{ }^{+} 319.25$ (calcd. 319.23).

\subsection{General Synthesis of the Hydrazone-Based Acrylate Monomers}

a)

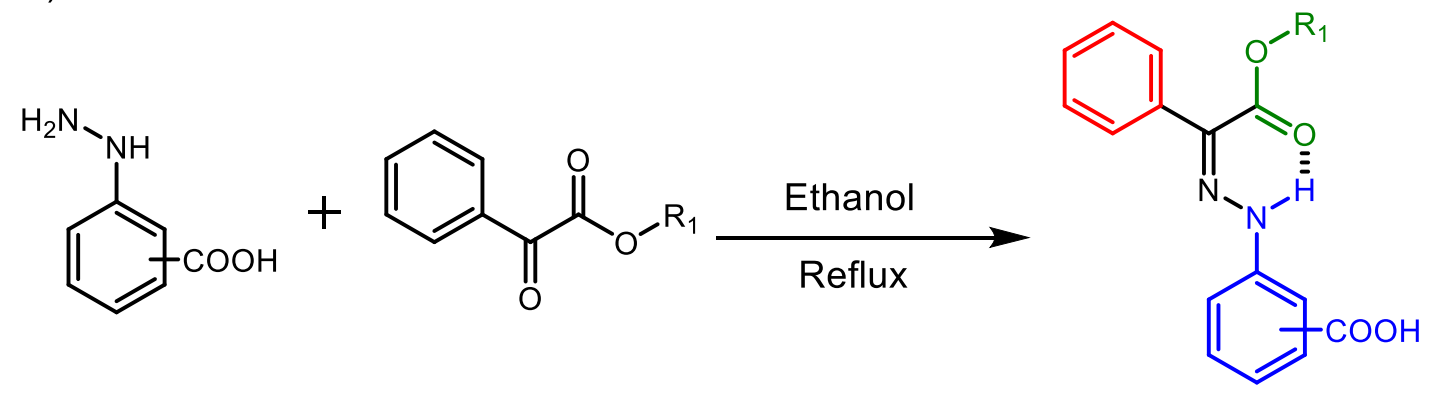

b)

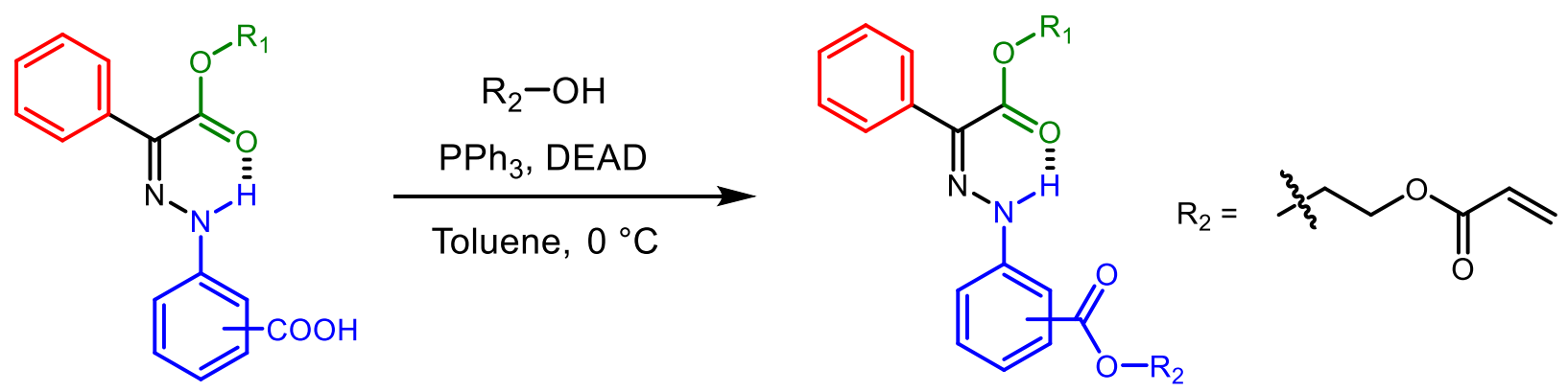

Scheme S3. General synthetic procedure for obtaining the hydrazone-based acrylates. 
a) Hydrazone precursor: The appropriate $\alpha$-keto ester (1 equiv.) and five drops of acetic acid were added to an ethanol solution of appropriate phenylhydrazine derivative (1 equiv.) and triethylamine (1 equiv.). The solution was refluxed for 3 hours, and excessive amount of water was then added to yield a precipitate, which was collected by filtration and washed with hexane and water. If precipitation did not occur, then the solution was extracted with DCM, and the organic layer was washed with water twice. The organic layer was dried over $\mathrm{Na}_{2} \mathrm{SO}_{4}$ and the solvent was removed under vacuum. The hydrazone monomer precursor was directly used in the next step without further purification.

b) Hydrazone-based acrylate monomer: DEAD ( $3 \mathrm{mmol})$ was added dropwise to a solution of the hydrazone-containing monomer precursor (1 equiv.), appropriate alcohol (1 equiv.) and $\mathrm{PPh}_{3}$ ( 1 equiv.) in toluene $(20 \mathrm{~mL})$ at $0{ }^{\circ} \mathrm{C}$. The reaction was then left to warm up to room temperature, and then stirred for an additional 20 minutes. After removal of the solvent under reduced pressure, the product was purified by column chromatography affording the hydrazone-containing monomer as a yellow solid.

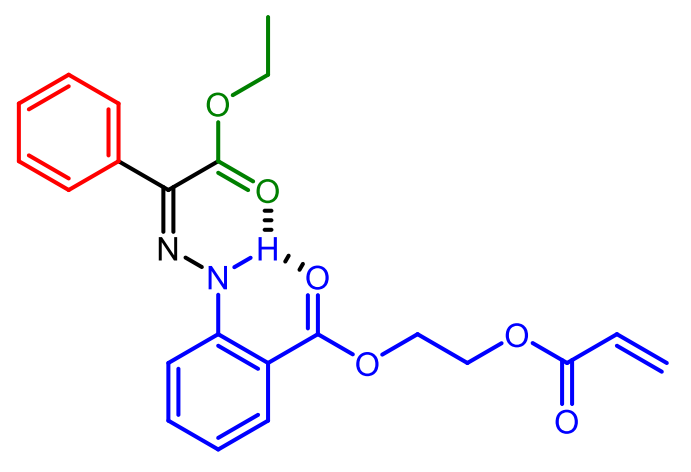

3

3: This compound was synthesized following the general procedure, while using ethyl phenylglyoxylate and 2-hydrazinobenzoic acid hydrochloride as starting materials. Acrylate 3 was obtained as a light-yellow solid with an overall yield of $57 \%$. m.p. $80.4-80.9{ }^{\circ} \mathrm{C} ;{ }^{1} \mathrm{H}$ NMR $(500$ $\left.\mathrm{MHz}, \mathrm{CD}_{2} \mathrm{Cl}_{2}\right) \delta 13.74(\mathrm{~s}, 1 \mathrm{H}), 8.01(\mathrm{dd}, J=8.0,1.5 \mathrm{~Hz}, 1 \mathrm{H}), 7.93(\mathrm{~d}, J=8.0 \mathrm{~Hz}, 1 \mathrm{H}), 7.71-7.66$ $(\mathrm{m}, 2 \mathrm{H}), 7.56-7.51(\mathrm{~m}, 1 \mathrm{H}), 7.43-7.32(\mathrm{~m}, 3 \mathrm{H}), 6.98(\mathrm{ddd}, J=8.2,7.2,1.2 \mathrm{~Hz}, 1 \mathrm{H}), 6.42(\mathrm{dd}$, $J=17.3,1.4 \mathrm{~Hz}, 1 \mathrm{H}), 6.16(\mathrm{dd}, J=17.3,10.4 \mathrm{~Hz}, 1 \mathrm{H}), 5.86(\mathrm{dd}, J=10.5,1.4 \mathrm{~Hz}, 1 \mathrm{H}), 4.63-4.59$ $(\mathrm{m}, 2 \mathrm{H}), 4.54-4.50(\mathrm{~m}, 2 \mathrm{H}), 4.42(\mathrm{q}, J=7.1 \mathrm{~Hz}, 2 \mathrm{H}), 1.38(\mathrm{t}, J=7.1 \mathrm{~Hz}, 3 \mathrm{H}) \mathrm{ppm} ;{ }^{13} \mathrm{C}$ NMR 
$\left(151 \mathrm{MHz}, \mathrm{CD}_{2} \mathrm{Cl}_{2}\right) \delta 167.51,166.35,163.09,146.76,137.07,135.18,132.43,131.64,131.60$, $129.15,128.65,128.54,128.46,120.99,115.11,112.82,63.37,62.76,62.06,14.55$ ppm. Hi-Res ESI-MS: $m / z$ found $\left[\mathrm{M}-\mathrm{H}^{+}\right]$for $\mathrm{C}_{22} \mathrm{H}_{23} \mathrm{~N}_{2} \mathrm{O}_{6}{ }^{+} 411.1555$ (calcd. 411.1556).

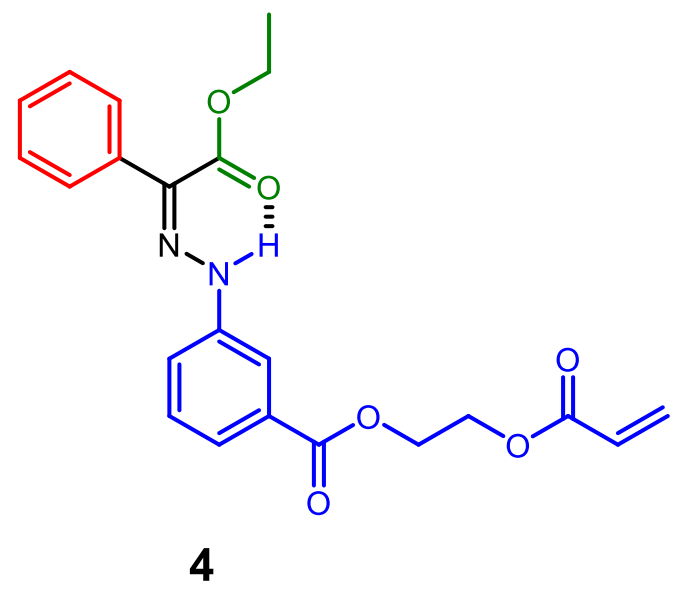

4: This compound was synthesized following the general procedure, while using ethyl phenylglyoxylate and 3-hydrazinobenzoic acid hydrochloride as starting materials. Acrylate $\mathbf{4}$ was obtained as a yellow oil with an overall yield of $60 \%$. ${ }^{1} \mathrm{H} \mathrm{NMR}\left(600 \mathrm{MHz}, \mathrm{CD}_{2} \mathrm{Cl}_{2}\right) \delta 12.41(\mathrm{~s}$, 1H), $7.89-7.84(\mathrm{~m}, 1 \mathrm{H}), 7.69-7.63(\mathrm{~m}, 3 \mathrm{H}), 7.54$ (dd, $J=8.1,1.3 \mathrm{~Hz}, 1 \mathrm{H}), 7.40$ (ddd, $J=11.4$, 10.0, $7.0 \mathrm{~Hz}, 3 \mathrm{H}), 7.37-7.32$ (m, 1H), 6.41 (dd, $J=17.3,1.4 \mathrm{~Hz}, 1 \mathrm{H}), 6.15$ (dd, $J=17.3,10.5$ $\mathrm{Hz}, 1 \mathrm{H}), 5.84$ (dd, $J=10.5,1.4 \mathrm{~Hz}, 1 \mathrm{H}), 4.54(\mathrm{dd}, J=5.8,3.4 \mathrm{~Hz}, 2 \mathrm{H}), 4.49$ (dd, $J=5.9,3.5 \mathrm{~Hz}$, $2 \mathrm{H}), 4.37$ (q, $J=7.1 \mathrm{~Hz}, 2 \mathrm{H}), 1.36(\mathrm{t}, J=7.1 \mathrm{~Hz}, 3 \mathrm{H}) \mathrm{ppm} .{ }^{13} \mathrm{C} \mathrm{NMR}\left(151 \mathrm{MHz}, \mathrm{CD}_{2} \mathrm{Cl}_{2}\right) \delta 165.97$, $165.78,163.56,143.68,136.33,131.14,131.02,129.49,129.44,128.65,128.06,127.87,127.76$,

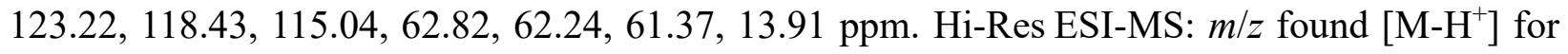
$\mathrm{C}_{22} \mathrm{H}_{23} \mathrm{~N}_{2} \mathrm{O}_{6}{ }^{+} 411.1556$ (calcd. 411.1556). 


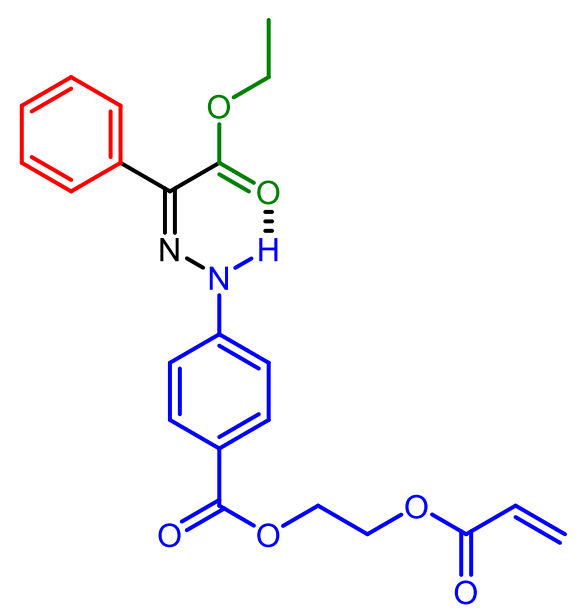

5

5: This compound was synthesized following the general procedure, while using ethyl phenylglyoxylate and 4-hydrazinobenzoic acid hydrochloride as starting materials. Acrylate $\mathbf{5}$ was obtained as a light-yellow solid with an overall yield $71 \%$ m.p. $76.8-77.6{ }^{\circ} \mathrm{C} ;{ }^{1} \mathrm{H}$ NMR $(600$ $\left.\mathrm{MHz}, \mathrm{CD}_{2} \mathrm{Cl}_{2}\right) \delta 12.41(\mathrm{~s}, 1 \mathrm{H}), 7.99(\mathrm{~d}, J=8.8 \mathrm{~Hz}, 2 \mathrm{H}), 7.69-7.60(\mathrm{~m}, 2 \mathrm{H}), 7.42-7.38(\mathrm{~m}, 2 \mathrm{H})$, $7.38-7.35(\mathrm{~m}, 1 \mathrm{H}), 7.31(\mathrm{~d}, J=8.7 \mathrm{~Hz}, 2 \mathrm{H}), 6.41(\mathrm{dd}, J=17.3,1.3 \mathrm{~Hz}, 1 \mathrm{H}), 6.16(\mathrm{dd}, J=17.4$, $10.5 \mathrm{~Hz}, 1 \mathrm{H}), 5.85(\mathrm{dd}, J=10.5,1.4 \mathrm{~Hz}, 1 \mathrm{H}), 4.51(\mathrm{dd}, J=6.3,3.0 \mathrm{~Hz}, 2 \mathrm{H}), 4.47$ (dd, $J=6.2,2.9$ $\mathrm{Hz}, 2 \mathrm{H}), 4.38$ (q, $J=7.1 \mathrm{~Hz}, 2 \mathrm{H}), 1.36(\mathrm{t}, J=7.1 \mathrm{~Hz}, 3 \mathrm{H}) \mathrm{ppm} .{ }^{13} \mathrm{C} \mathrm{NMR}\left(151 \mathrm{MHz}, \mathrm{CD}_{2} \mathrm{Cl}_{2}\right) \delta$ 13C NMR (151 MHz, CD2Cl2) $\delta 166.41,166.37,163.99,147.81,136.64,131.92,131.52,131.43$, 129.26, 128.68, 128.62, 128.48, 123.89, 114.03, 62.97, 62.94, 62.16, 14.46 ppm. Hi-Res ESI-MS: $m / z$ found $\left[\mathrm{M}-\mathrm{H}^{+}\right]$for $\mathrm{C}_{22} \mathrm{H}_{23} \mathrm{~N}_{2} \mathrm{O}_{6}{ }^{+} 411.1545$ (calcd. 411.1556).

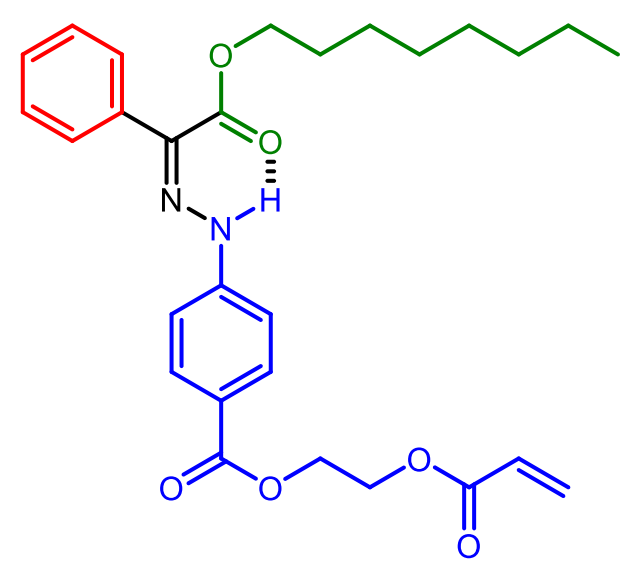

6 
6: This compound was synthesized following the general procedure, while using $n$-octyl phenylglyoxylate and 4-hydrazinobenzoic acid as starting materials. Acrylate $\mathbf{6}$ was obtained as a light-yellow solid with an overall yield of $67 \%$. m.p. $67.5-68.5{ }^{\circ} \mathrm{C} ;{ }^{1} \mathrm{H}$ NMR (600 MHz, $\mathrm{CD}_{2} \mathrm{Cl}_{2}$ ) $\delta 12.41(\mathrm{~s}, 1 \mathrm{H}), 7.99(\mathrm{~d}, J=8.7 \mathrm{~Hz}, 2 \mathrm{H}), 7.68-7.63(\mathrm{~m}, 2 \mathrm{H}), 7.43-7.33(\mathrm{~m}, 3 \mathrm{H}), 7.30(\mathrm{~d}, J=8.7$ $\mathrm{Hz}, 2 \mathrm{H}), 6.41$ (dd, $J=17.3,1.3 \mathrm{~Hz}, 1 \mathrm{H}), 6.16$ (dd, $J=17.3,10.5 \mathrm{~Hz}, 1 \mathrm{H}), 5.85$ (dd, $J=10.4,1.3$ $\mathrm{Hz}, 1 \mathrm{H}), 4.51(\mathrm{dd}, J=6.3,3.1 \mathrm{~Hz}, 2 \mathrm{H}), 4.47(\mathrm{dd}, J=6.4,3.1 \mathrm{~Hz}, 2 \mathrm{H}), 4.31(\mathrm{t}, J=6.7 \mathrm{~Hz}, 2 \mathrm{H})$, $1.77-1.66(\mathrm{~m}, 2 \mathrm{H}), 1.37(\mathrm{dd}, J=15.0,7.2 \mathrm{~Hz}, 2 \mathrm{H}), 1.33-1.23(\mathrm{~m}, 8 \mathrm{H}), 0.88(\mathrm{t}, J=7.0 \mathrm{~Hz}, 3 \mathrm{H})$ ppm; ${ }^{13} \mathrm{C}$ NMR $\left(151 \mathrm{MHz}, \mathrm{CD}_{2} \mathrm{Cl}_{2}\right) \delta 166.41,166.37,164.07,147.82,136.65,131.92,131.53$, $131.51,129.26,128.68,128.63,128.45,123.87,114.02,66.25,62.97,62.95,32.30,29.70,29.65$, 28.94, 26.54, 23.20, $14.42 \mathrm{ppm}$. Hi-Res ESI-MS: $m / z$ found $\left[\mathrm{M}-\mathrm{H}^{+}\right]$for $\mathrm{C}_{28} \mathrm{H}_{35} \mathrm{~N}_{2} \mathrm{O}_{6}{ }^{+} 495.2480$ (calcd. 495.2495).

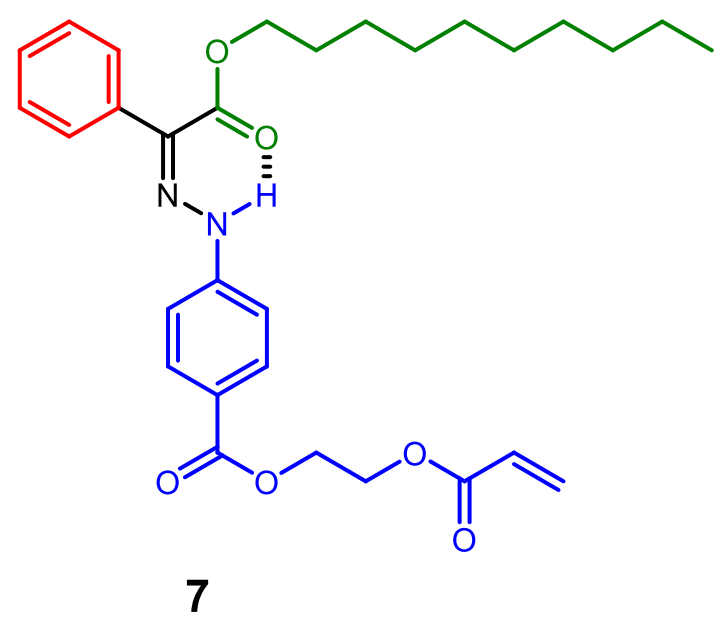

7: This compound was synthesized following the general procedure, while using $n$-decyl phenylglyoxylate and 4-hydrazinobenzoic acid as starting materials. Acrylate $\mathbf{7}$ was obtained as a light yellow solid with an overall yield of $70 \%$ m.p. $58.1-58.6{ }^{\circ} \mathrm{C} ;{ }^{1} \mathrm{H} \mathrm{NMR}\left(600 \mathrm{MHz}, \mathrm{CD}_{2} \mathrm{Cl}_{2}\right)$ $\delta 12.41(\mathrm{~s}, 1 \mathrm{H}), 7.99(\mathrm{~d}, J=8.8 \mathrm{~Hz}, 2 \mathrm{H}), 7.70-7.62(\mathrm{~m}, 2 \mathrm{H}), 7.43-7.34(\mathrm{~m}, 3 \mathrm{H}), 7.33-7.27(\mathrm{~m}$, 2H), $6.41(\mathrm{dd}, J=17.3,1.4 \mathrm{~Hz}, 1 \mathrm{H}), 6.16(\mathrm{dd}, J=17.3,10.5 \mathrm{~Hz}, 1 \mathrm{H}), 5.85(\mathrm{dd}, J=10.5,1.4 \mathrm{~Hz}$, 1H), $4.53-4.49$ (m, 2H), $4.49-4.45$ (m, 2H), 4.31 (t, $J=6.7 \mathrm{~Hz}, 2 \mathrm{H}), 1.77-1.67$ (m, 2H), 1.42 $-1.35(\mathrm{~m}, 2 \mathrm{H}), 1.33-1.23(\mathrm{~m}, 12 \mathrm{H}), 0.88(\mathrm{t}, J=7.0 \mathrm{~Hz}, 3 \mathrm{H}) \mathrm{ppm} ;{ }^{13} \mathrm{C} \mathrm{NMR}\left(151 \mathrm{MHz}, \mathrm{CD}_{2} \mathrm{Cl}_{2}\right)$ $\delta 166.41,166.37,164.07,147.82,136.65,131.92,131.53,131.50,129.26,128.69,128.63,128.45$, $123.87,114.02,66.25,62.97,62.95,32.46,30.05,29.87,29.69,28.95,26.55,23.25,14.45 \mathrm{ppm}$. Hi-Res ESI-MS: $m / z$ found $\left[\mathrm{M}-\mathrm{H}^{+}\right]$for $\mathrm{C}_{30} \mathrm{H}_{39} \mathrm{~N}_{2} \mathrm{O}_{6}{ }^{+} 523.2797$ (calcd. 523.2808). 


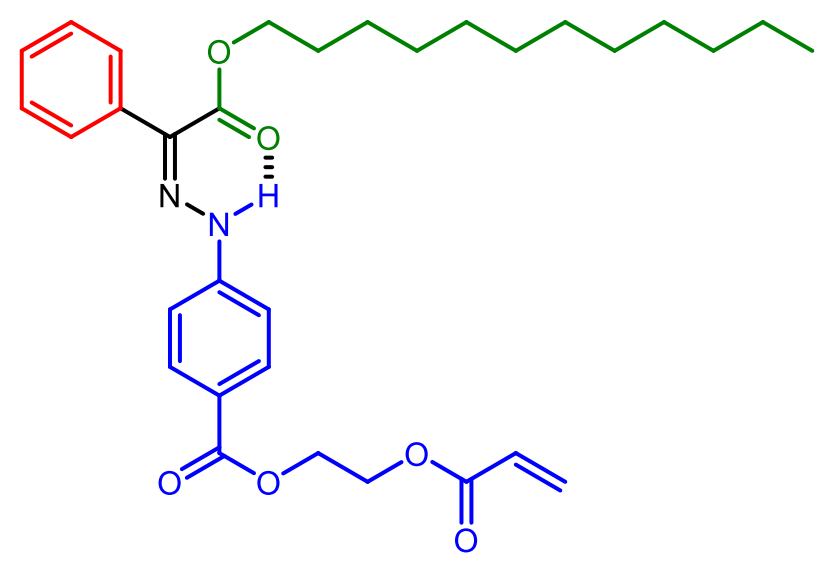

8

8: This compound was synthesized following the general procedure, while using $n$-dodecyl phenylglyoxylate and 4-hydrazinobenzoic acid as starting materials. Acrylate $\mathbf{8}$ was obtained as a light-yellow solid with an overall yield of $60 \%$. m.p. $55.0-56.1^{\circ} \mathrm{C} ;{ }^{1} \mathrm{H}$ NMR (600 MHz, $\mathrm{CD}_{2} \mathrm{Cl}_{2}$ ) $\delta 12.42(\mathrm{~s}, 1 \mathrm{H}), 7.99(\mathrm{~d}, J=8.8 \mathrm{~Hz}, 2 \mathrm{H}), 7.69-7.63(\mathrm{~m}, 2 \mathrm{H}), 7.43-7.34(\mathrm{~m}, 3 \mathrm{H}), 7.33-7.28(\mathrm{~m}$, 2H), $6.41(\mathrm{dd}, J=17.3,1.4 \mathrm{~Hz}, 1 \mathrm{H}), 6.16(\mathrm{dd}, J=17.3,10.5 \mathrm{~Hz}, 1 \mathrm{H}), 5.85(\mathrm{dd}, J=10.5,1.4 \mathrm{~Hz}$, 1H), $4.53-4.49$ (m, 2H), $4.49-4.45$ (m, 2H), 4.31 (t, $J=6.7 \mathrm{~Hz}, 2 \mathrm{H}), 1.75-1.68(\mathrm{~m}, 2 \mathrm{H}), 1.41$ $-1.35(\mathrm{~m}, 2 \mathrm{H}), 1.33-1.23(\mathrm{~m}, 16 \mathrm{H}), 0.88(\mathrm{t}, J=7.0 \mathrm{~Hz}, 3 \mathrm{H}) \mathrm{ppm} ;{ }^{13} \mathrm{C} \mathrm{NMR}\left(151 \mathrm{MHz}, \mathrm{CD}_{2} \mathrm{Cl}_{2}\right)$ $\delta 166.41,166.37,164.08,147.82,136.65,131.92,131.53,131.51,129.26,128.69,128.63,128.45$, 123.88, 114.02, 66.26, 62.97, 62.95, 32.50, 30.21, 30.20, 30.09, 30.04, 29.92, 29.69, 28.95, 26.55, 23.26, 14.45 ppm. Hi-Res ESI-MS: $m / z$ found $\left[\mathrm{M}-\mathrm{H}^{+}\right]$for $\mathrm{C}_{32} \mathrm{H}_{43} \mathrm{~N}_{2} \mathrm{O}_{6}{ }^{+} 551.3112$ (calcd. 551.3121 ).

\subsection{General Polymerization Procedure:}
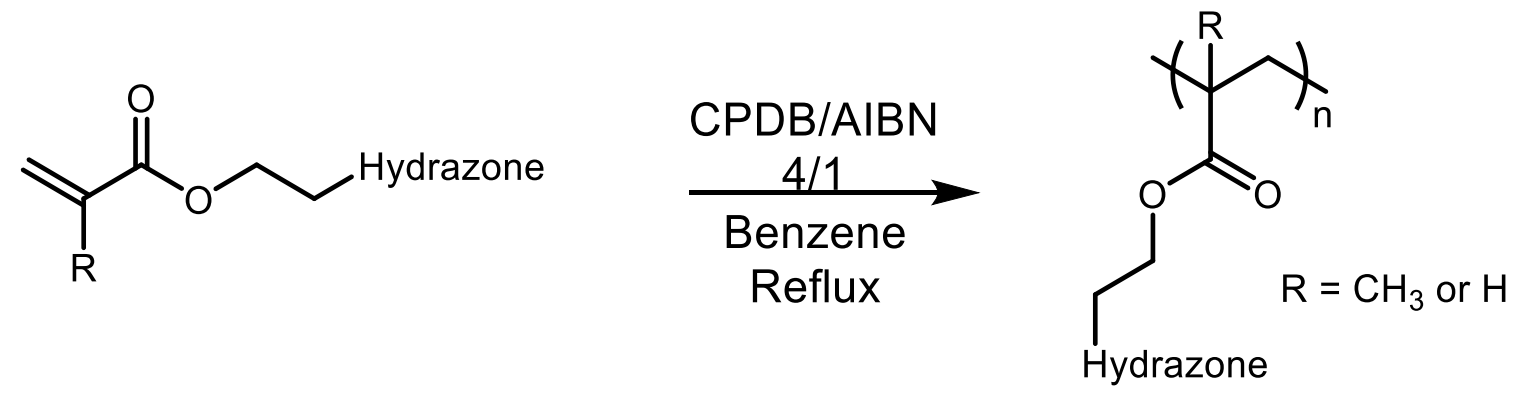

Scheme S4. Summary of the polymerization route employed to achieve the photochromic hydrazone polymers. 
A stock solution of 2-cyano-2-propyl benzodithioate (RAFT agent; $14.79 \mathrm{mg}$ ) and azobisisobutyronitrile (initiator; $2.86 \mathrm{mg}$ ) was prepared by dissolving them in $10.00 \mathrm{~mL}$ distilled benzene. This solution was used in all the polymerization reactions.

The appropriate monomer (1.00 mmol, 150 eq.) was added to a $10 \mathrm{~mL}$ Schlenk tube along with a stir bar. A stock solution $\left(1.00 \mathrm{~mL} ; 6.68 \times 10^{-3} \mathrm{mmol} ., 1\right.$ eq. CPBD; $1.74 \times 10^{-3} \mathrm{mmol}$., 0.26 eq. AIBN) was then added to the flask and the reaction solution was freeze/pump/thawed three times and backfilled with $\mathrm{N}_{2}$. The flask was then heated at $80{ }^{\circ} \mathrm{C}$ for $24 \mathrm{~h}$. The reaction mixture was then allowed to cool to room temperature before diluting slightly with dichloromethane to dissolve any precipitate. The product was then precipitated from cold methanol, filtered, and dried. The pale-yellow solids were then stirred in $50{ }^{\circ} \mathrm{C}$ ethanol for several hours, filtered, and washed with ethanol and methanol. The polymers were recovered as fine pale-yellow powders.

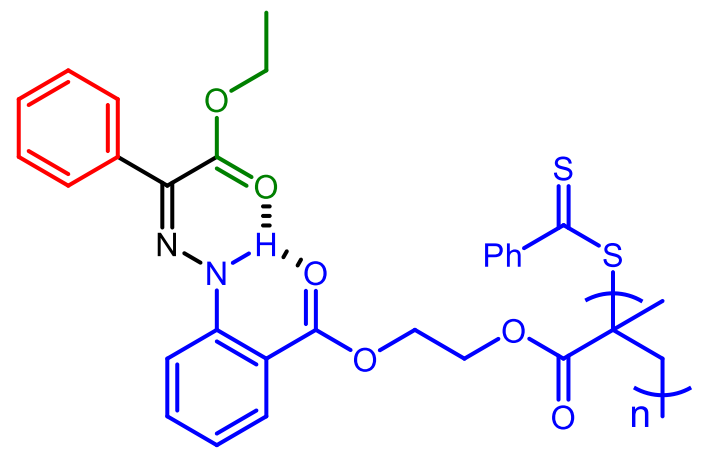

\section{P1}

P1: This compound was synthesized following the general procedure, while using $\mathbf{1}$ as starting material; yield $58 \% .{ }^{1} \mathrm{H}$ NMR $\left(\mathrm{CD}_{2} \mathrm{Cl}_{2}, 600 \mathrm{MHz}\right) \delta 13.57,7.95-6.78,4.50-4.07,2.12-0.89$ ppm. $\mathrm{M}_{\mathrm{n}}(\mathrm{GPC})=2.8 \times 10^{4} \mathrm{~g} / \mathrm{mol}$ and $\mathrm{Ð}=1.14$.

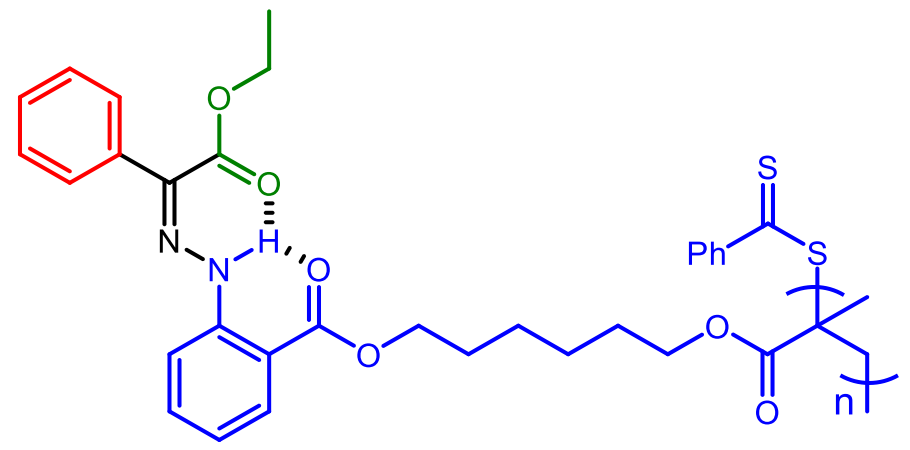

P2 
P2: This compound was synthesized following the general procedure, while using 2 as starting material; yield $69 \%$. ${ }^{1} \mathrm{H} \mathrm{NMR}\left(\mathrm{CD}_{2} \mathrm{Cl}_{2}, 600 \mathrm{MHz}\right) \delta 13.70,7.95-6.83,4.50-3.85,1.80-0.78$ ppm. $\mathrm{M}_{\mathrm{n}}(\mathrm{GPC})=3.1 \times 10^{4} \mathrm{~g} / \mathrm{mol}$ and $\mathrm{Ð}=1.27$.

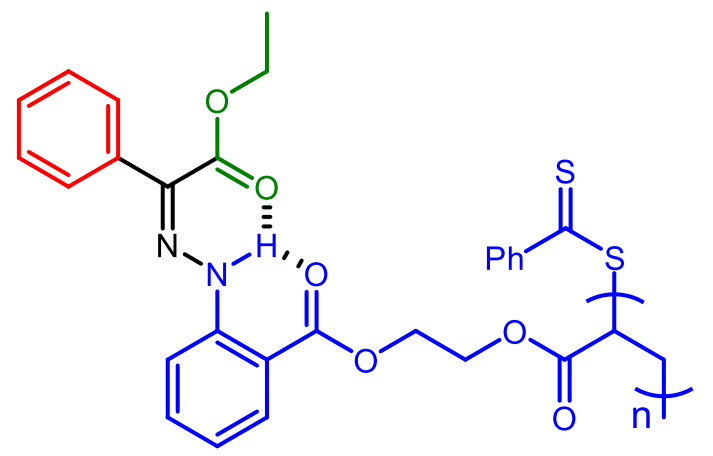

P3

P3: This compound was synthesized following the general procedure, while using 7 as starting material; yield: $42 \% .{ }^{1} \mathrm{H}$ NMR (600 $\left.\mathrm{MHz} \mathrm{CD}_{2} \mathrm{Cl}_{2}\right) \delta 13.55,7.83,7.70,7.54,7.40-7.20$, $6.79,4.35-3.95,2.56-0.80 \mathrm{ppm} . \mathrm{Mn}_{\mathrm{n}}(\mathrm{GPC})=1.3 \times 10^{4} \mathrm{~g} / \mathrm{mol}$ and $\mathrm{Ð}=1.55$.

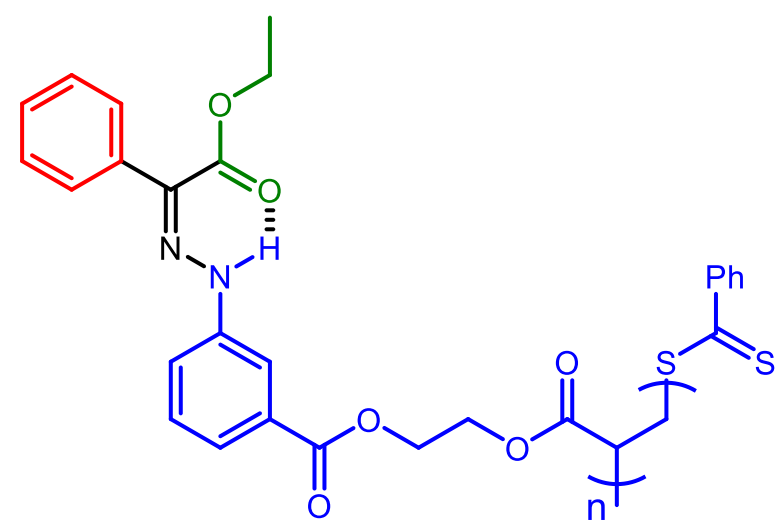

P4

P4: This compound was synthesized following the general procedure, while using $\mathbf{8}$ as starting material and heated for 4 days; yield: $41 \%$. ${ }^{1} \mathrm{H}$ NMR $\left(600 \mathrm{MHz}, \mathrm{CD}_{2} \mathrm{Cl}_{2}\right) \delta 12.22,7.66,7.60-$ $7.44,7.40-7.15,4.45-4.15,2.46-0.80 \mathrm{ppm} . \mathrm{M}_{\mathrm{n}}(\mathrm{GPC})=9.4 \times 10^{3} \mathrm{~g} / \mathrm{mol}$ and $Đ=1.37$. 


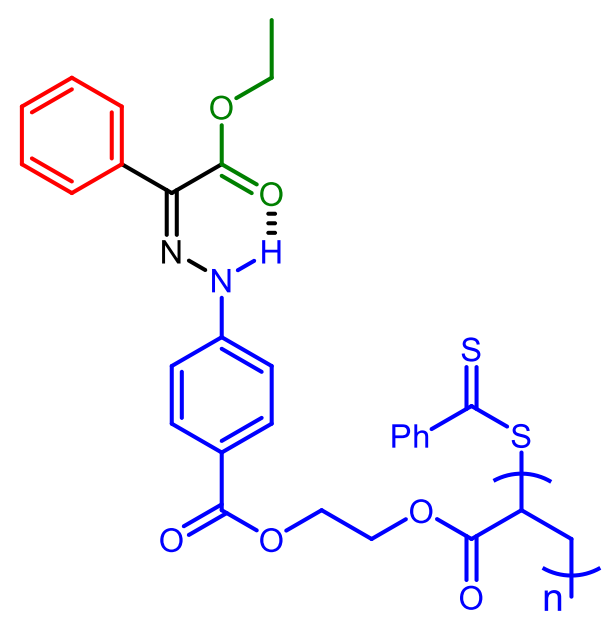

P5

P5: This compound was synthesized following the general procedure, while using $\mathbf{9}$ as starting material; yield: $64 \%$. ${ }^{1} \mathrm{H}$ NMR (600 MHz, $\left.\mathrm{CD}_{2} \mathrm{Cl}_{2}\right) \delta 12.20,7.83,7.54,7.27,7.10,4.35-4.15$, $2.50-0.80 \mathrm{ppm} . \mathrm{M}_{\mathrm{n}}(\mathrm{GPC})=1.3 \times 10^{4} \mathrm{~g} / \mathrm{mol}$ and $\mathrm{Ð}=1.40$.

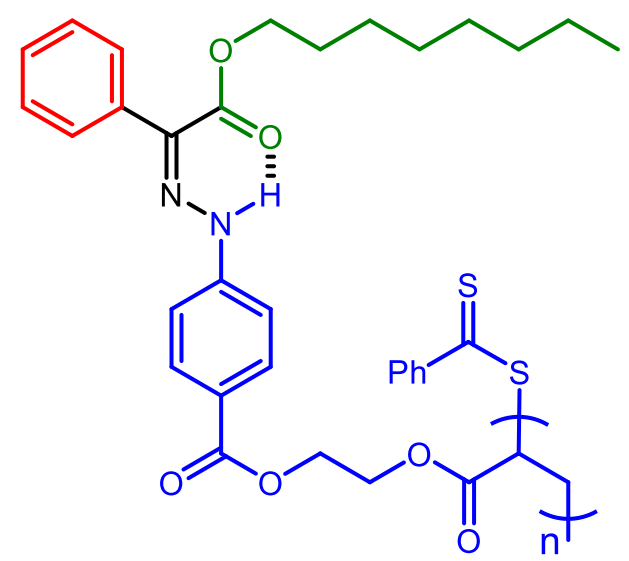

P6

P6: This compound was synthesized following the general procedure, while using $\mathbf{1 1}$ as starting material; yield: $50 \%$. ${ }^{1} \mathrm{H}$ NMR (600 MHz, $\left.\mathrm{CD}_{2} \mathrm{Cl}_{2}\right) \delta 12.23,7.84,7.54,7.20,7.09,4.41-4.09$, $1.73-1.16 \mathrm{ppm} . \mathrm{M}_{\mathrm{n}}(\mathrm{GPC})=1.4 \times 10^{4} \mathrm{~g} / \mathrm{mol}$ and $\mathrm{Ð}=1.63$. 


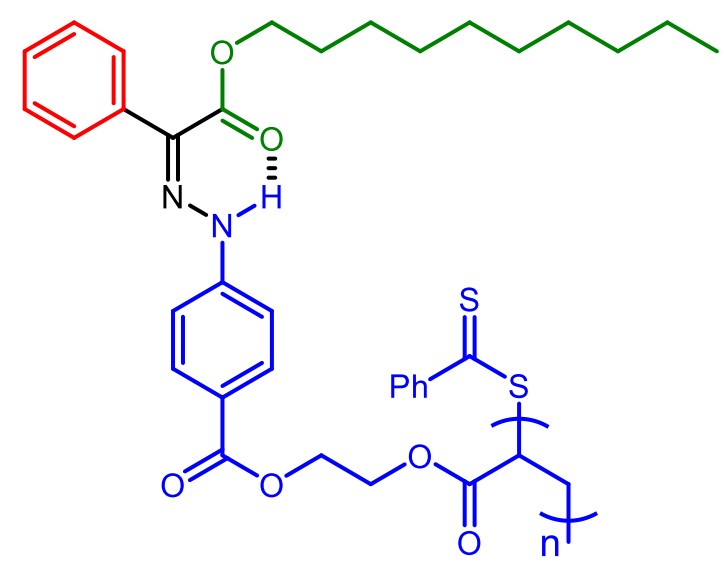

P7

P7: This compound was synthesized following the general procedure, while using 12 as starting material; yield: $51 \%$. ${ }^{1} \mathrm{H}$ NMR (600 MHz, $\left.\mathrm{CD}_{2} \mathrm{Cl}_{2}\right) \delta 12.23,7.84,7.54,7.26,7.09,4.45$ - 4.05, $1.75-1.09 \mathrm{ppm} . \mathrm{M}_{\mathrm{n}}(\mathrm{GPC})=1.7 \times 10^{4} \mathrm{~g} / \mathrm{mol}$ and $Đ=1.21$.

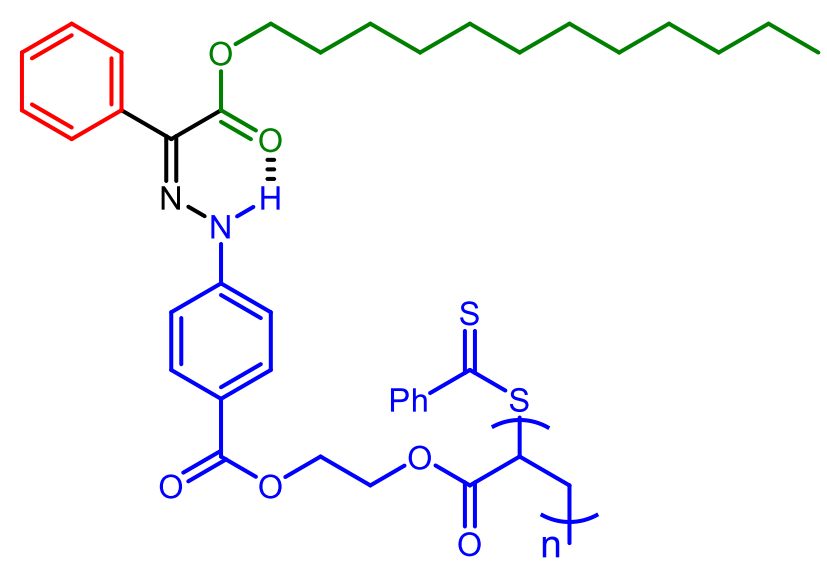

P8

P8: This compound was synthesized following the general procedure, while using 13 as starting material; yield: $47 \%$. ${ }^{1} \mathrm{H}$ NMR $\left(600 \mathrm{MHz}, \mathrm{CD}_{2} \mathrm{Cl}_{2}\right) \delta 12.23,7.84,7.54,7.27,7.09,4.42$ - 4.06, $1.72-1.14 \mathrm{ppm} . \mathrm{M}_{\mathrm{n}}(\mathrm{GPC})=1.4 \times 10^{4} \mathrm{~g} / \mathrm{mol}$ and $\mathrm{Ð}=1.47$. 
a)

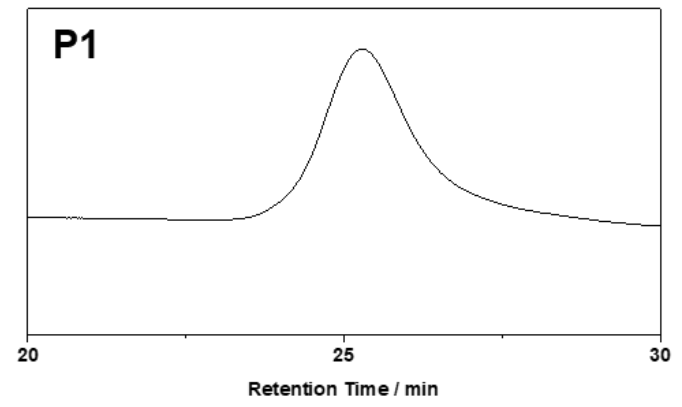

b)

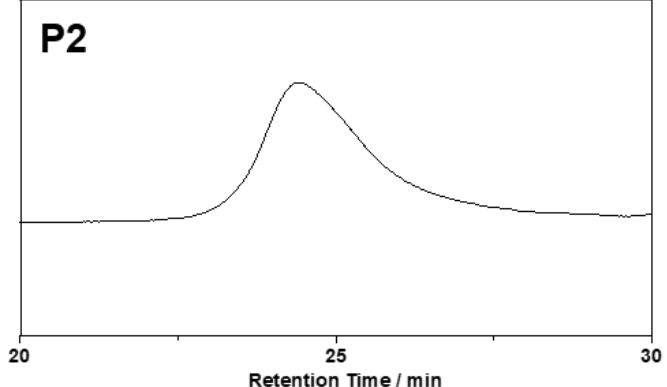

c)

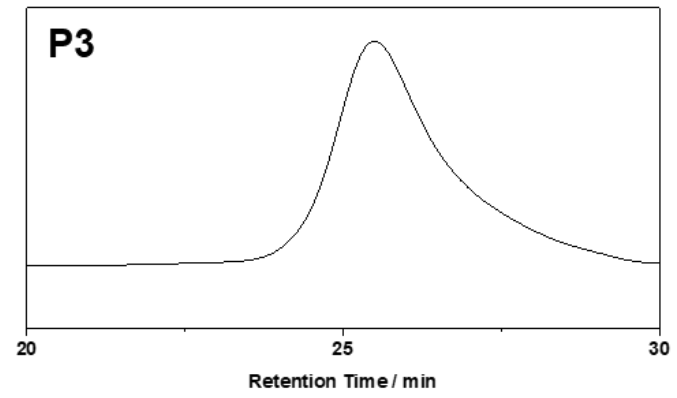

d)

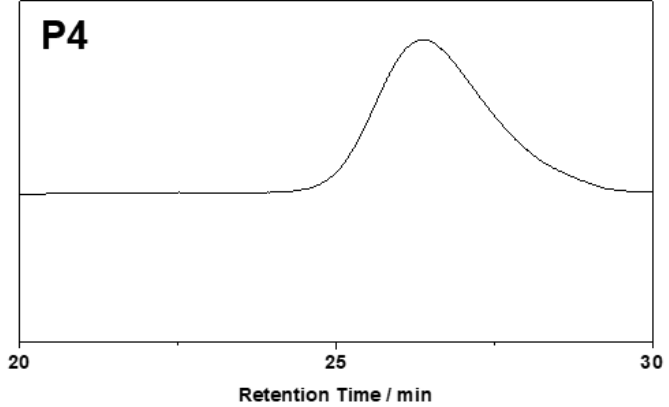

e)

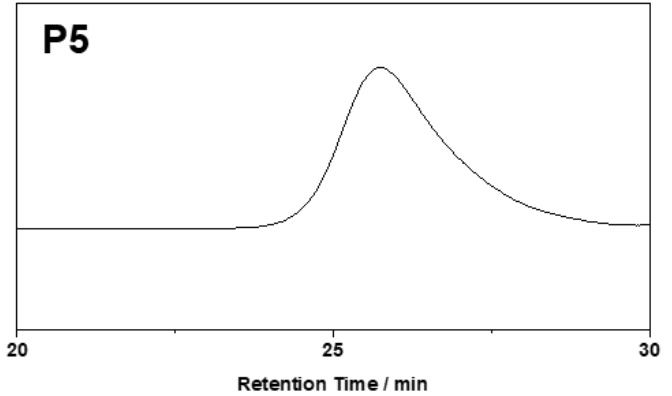

f)

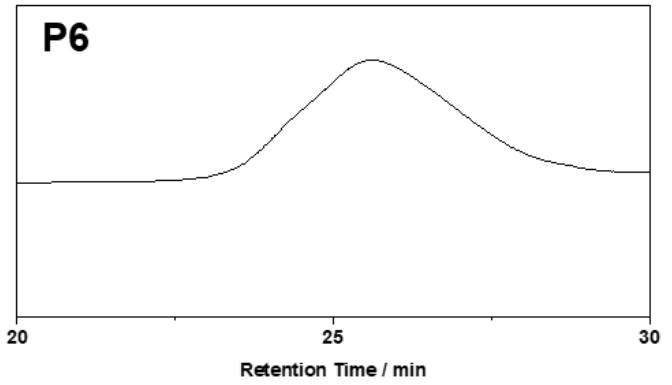

g)

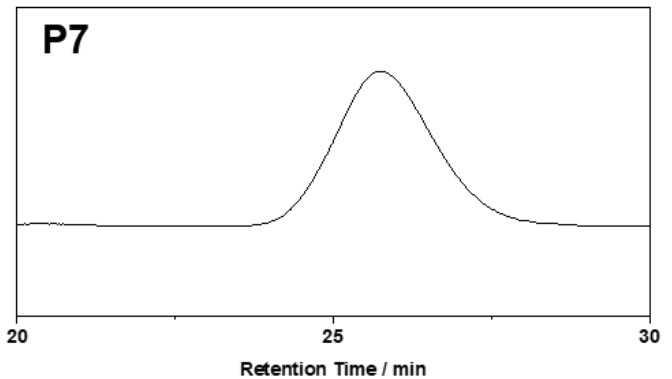

h)

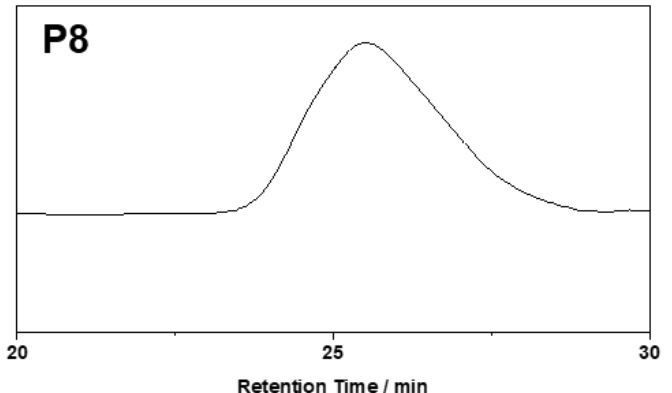

Figure S1 GPC chromatograms of polymers P1-P8. The molecular weight and polydispersity data are summarized in Table $\mathbf{S 1 .}$ 
Table S1 Summary of number average molecular weight $\mathrm{M}_{\mathrm{n}}$ (From gel permeation chromatography) and dispersity $\left(Đ, \mathrm{M}_{\mathrm{w}} / \mathrm{M}_{\mathrm{n}}\right)$

\begin{tabular}{ccc}
\hline Polymer & $\mathrm{M}_{\mathrm{n}}$ & $Ð, \mathrm{M}_{\mathrm{w}} / \mathrm{M}_{\mathrm{n}}$ \\
\hline P1 & $2.8 \times 10^{4}$ & 1.14 \\
P2 & $3.1 \times 10^{4}$ & 1.27 \\
P3 & $1.3 \times 10^{4}$ & 1.55 \\
P4 & $0.94 \times 10^{4}$ & 1.37 \\
P5 & $1.3 \times 10^{4}$ & 1.40 \\
P6 & $1.4 \times 10^{4}$ & 1.63 \\
P7 & $1.7 \times 10^{4}$ & 1.21 \\
P8 & $1.4 \times 10^{4}$ & 1.47 \\
\hline
\end{tabular}

\subsection{Synthesis of model compounds for thermal half-life measurements:}
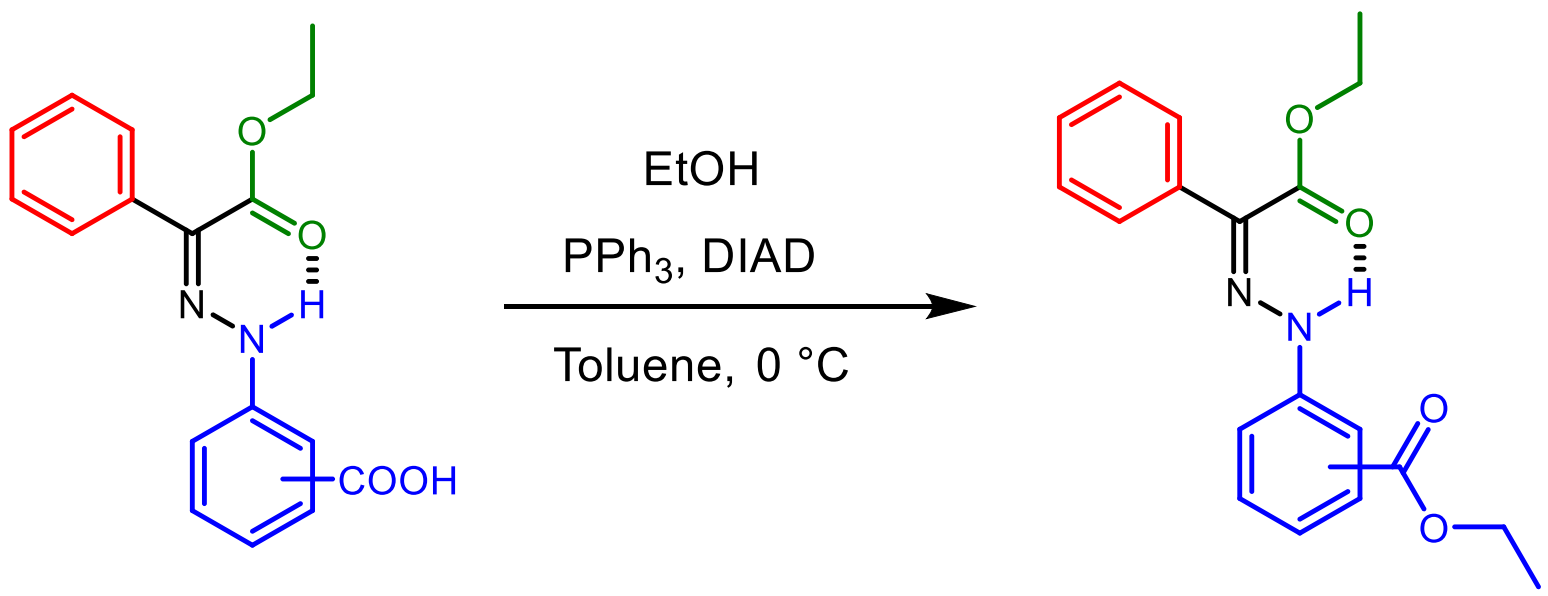

Scheme S5. General synthetic procedure for obtaining the model compounds used in the thermal half-life measurements.

Diisopropyl azodicarboxylate $(3 \mathrm{mmol})$ was added dropwise to a solution of the hydrazonecontaining monomer precursor (1 equiv.), ethanol (1 equiv.) and $\mathrm{PPh}_{3}$ (1 equiv.) in toluene $(20 \mathrm{~mL})$ at $0{ }^{\circ} \mathrm{C}$. The reaction was then left to warm up to room temperature, and then stirred for an additional 20 minutes. After removal of the solvent under reduced pressure, the product was 
purified by column chromatography affording the hydrazone-containing monomer as a yellow solid.<smiles></smiles>

\section{3}

13: This compound was synthesized, and its identity confirmed by comparing its ${ }^{1} \mathrm{H}$ NMR spectrum with that published in the literature. ${ }^{\mathrm{S} 1}{ }^{1} \mathrm{H} \mathrm{NMR}\left(500 \mathrm{MHz}, \mathrm{CD}_{2} \mathrm{Cl}_{2}\right) \delta 13.79(\mathrm{~s}, 1 \mathrm{H}), 8.02$ (dd, $J=8.0,1.5 \mathrm{~Hz}, 1 \mathrm{H}), 7.94-7.90(\mathrm{~m}, 1 \mathrm{H}), 7.72-7.67(\mathrm{~m}, 2 \mathrm{H}), 7.54-7.49(\mathrm{~m}, 1 \mathrm{H}), 7.43-$ 7.32 (m, 3H), 6.97 (ddd, $J=8.1,7.2,1.2 \mathrm{~Hz}, 1 \mathrm{H}), 4.42$ (qd, $J=7.1,2.9 \mathrm{~Hz}, 4 \mathrm{H}), 1.40$ (dt, $J=20.3$, $7.1 \mathrm{~Hz}, 6 \mathrm{H}) \mathrm{ppm}$.

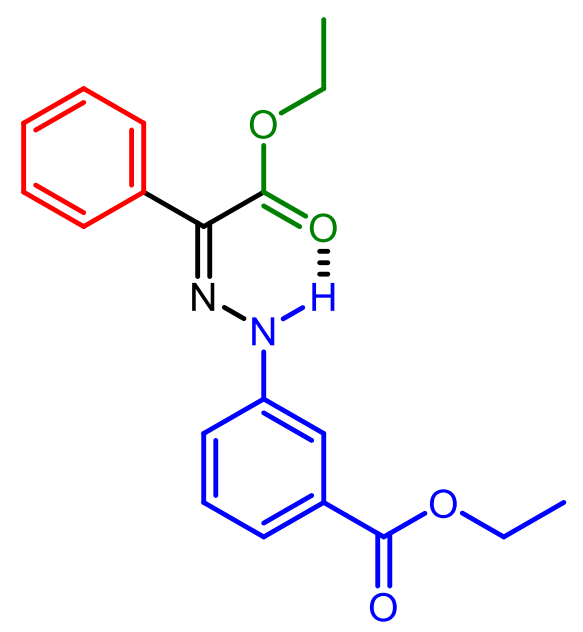

14

14: This compound was synthesized following the general procedure, while using ethyl phenylglyoxylate and 3-hydrazinobenzoic acid hydrochloride as starting materials. Hydrazone 14 was obtained as a yellow solid with an overall yield of $81 \%$. m.p. $79.5-80.3{ }^{\circ} \mathrm{C}$. ${ }^{1} \mathrm{H}$ NMR $(500$ $\left.\mathrm{MHz}, \mathrm{CD}_{2} \mathrm{Cl}_{2}\right) \delta 12.42(\mathrm{~s}, 1 \mathrm{H}), 7.88-7.83(\mathrm{~m}, 1 \mathrm{H}), 7.65$ (ddd, $\left.J=9.3,3.1,1.5 \mathrm{~Hz}, 3 \mathrm{H}\right), 7.53$ (ddd, 
$J=8.1,2.3,1.0 \mathrm{~Hz}, 1 \mathrm{H}), 7.45-7.37(\mathrm{~m}, 3 \mathrm{H}), 7.37-7.32(\mathrm{~m}, 1 \mathrm{H}), 4.42-4.30(\mathrm{~m}, 4 \mathrm{H}), 1.37(\mathrm{dt}$, $J=14.6,7.1 \mathrm{~Hz}, 6 \mathrm{H}) \mathrm{ppm} ;{ }^{13} \mathrm{C} \mathrm{NMR}\left(151 \mathrm{MHz}, \mathrm{CD}_{2} \mathrm{Cl}_{2}\right) \delta 166.73,164.14,144.18,136.96,132.41$, 129.97, 129.85, 129.23, 128.43, 128.30, 123.67, 118.65, 115.54, 61.91, 61.63, 14.67, 14.49 ppm. Hi-Res ESI-MS: $m / z$ found $\left[\mathrm{M}-\mathrm{H}^{+}\right]$for $\mathrm{C}_{19} \mathrm{H}_{21} \mathrm{~N}_{2} \mathrm{O}_{4}{ }^{+} 341.1489$ (calcd. 341.1501).

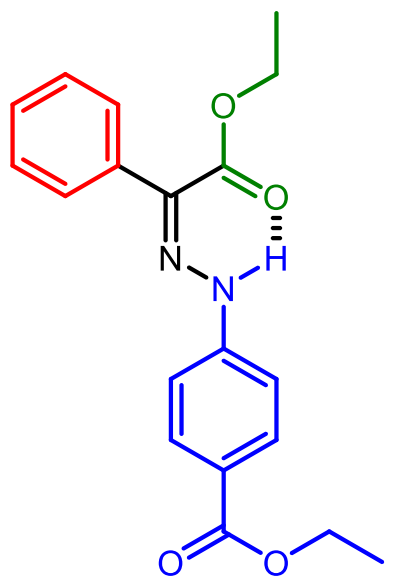

15

15: This compound was synthesized following the general procedure, while using ethyl phenylglyoxylate and 4-hydrazinobenzoic acid hydrochloride as starting materials. Hydrazone 15 was obtained as a yellow solid with an overall yield of $66 \%$. m.p. $116.8-117.4{ }^{\circ} \mathrm{C} .{ }^{1} \mathrm{H}$ NMR $(500$ $\left.\mathrm{MHz}, \mathrm{CD}_{2} \mathrm{Cl}_{2}\right) \delta 12.42(\mathrm{~s}, 1 \mathrm{H}), 7.99(\mathrm{~d}, J=8.8 \mathrm{~Hz}, 2 \mathrm{H}), 7.71-7.62(\mathrm{~m}, 2 \mathrm{H}), 7.43-7.33(\mathrm{~m}, 3 \mathrm{H})$, $7.30(\mathrm{~d}, J=8.8 \mathrm{~Hz}, 2 \mathrm{H}), 4.35$ (dq, $J=27.0,7.1 \mathrm{~Hz}, 4 \mathrm{H}), 1.36$ (td, $J=7.1,5.2 \mathrm{~Hz}, 6 \mathrm{H}) \mathrm{ppm} ;{ }^{13} \mathrm{C}$ NMR $\left(151 \mathrm{MHz}, \mathrm{CD}_{2} \mathrm{Cl}_{2}\right) \delta 166.65,164.00,147.52,136.70,131.70,131.20,129.26,128.58$,

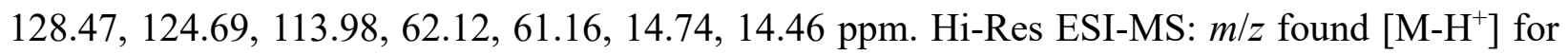
$\mathrm{C}_{19} \mathrm{H}_{21} \mathrm{~N}_{2} \mathrm{O}_{4}{ }^{+} 341.1492$ (calcd. 341.1501). 


\section{NMR Spectroscopy Characterization}

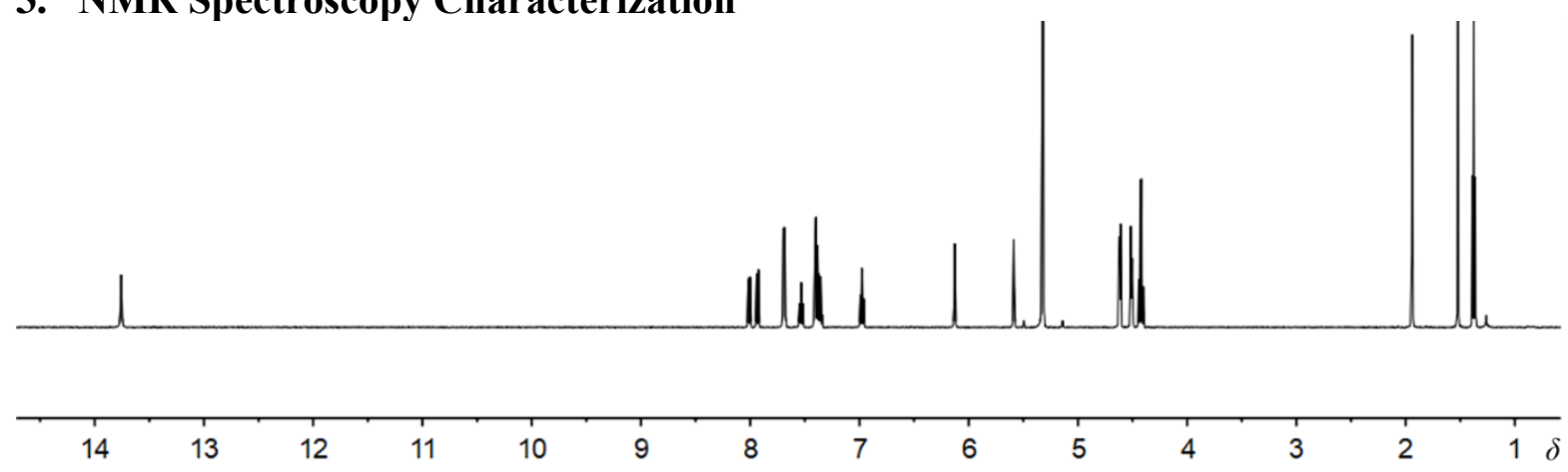

Figure S2. ${ }^{1} \mathrm{H}$ NMR spectrum of 1 in $\mathrm{CD}_{2} \mathrm{Cl}_{2}$ at $298 \mathrm{~K}$

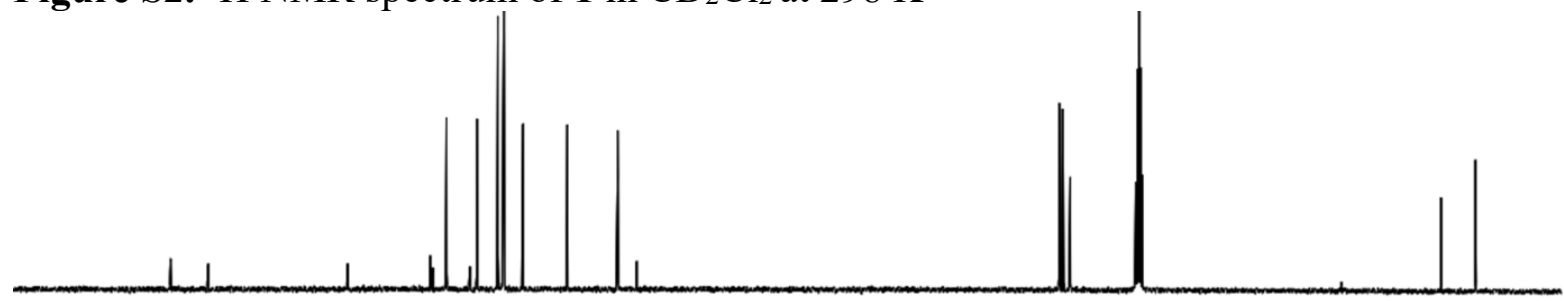

180

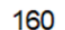

120

100

$80 \quad 60$

40

20

Figure S3. ${ }^{13} \mathrm{C}$ NMR spectrum of 1 in $\mathrm{CD}_{2} \mathrm{Cl}_{2}$ at $298 \mathrm{~K}$

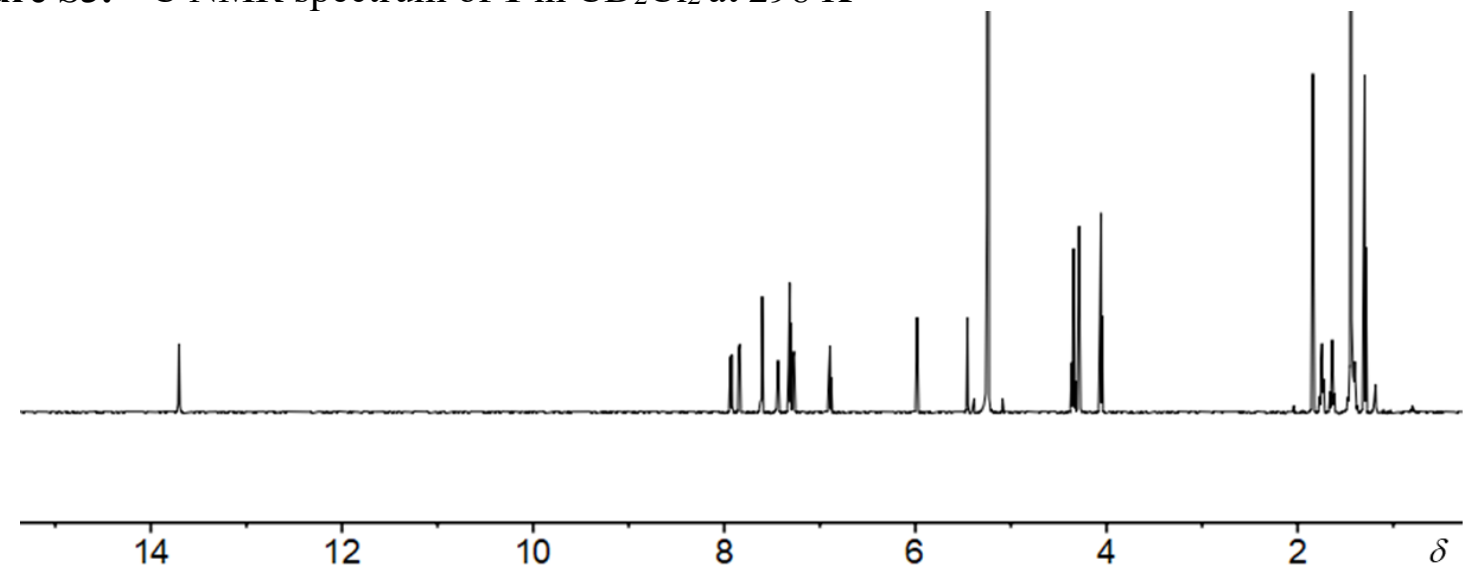

Figure S4. ${ }^{1} \mathrm{H}$ NMR spectrum of 2 in $\mathrm{CD}_{2} \mathrm{Cl}_{2}$ at $298 \mathrm{~K}$ 


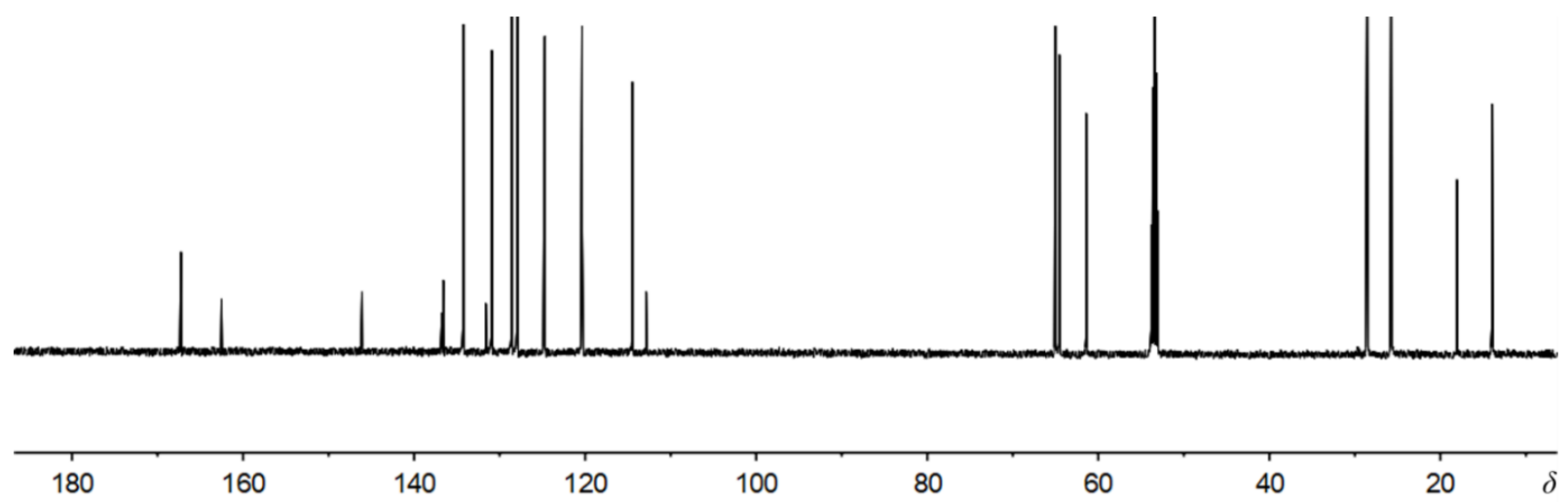

Figure S5. ${ }^{13} \mathrm{C}$ NMR spectrum of 2 in $\mathrm{CD}_{2} \mathrm{Cl}_{2}$ at $298 \mathrm{~K}$

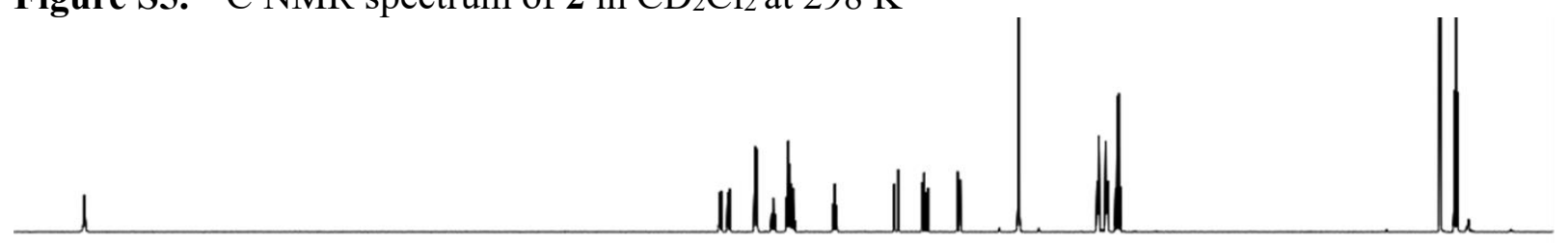

\begin{tabular}{llllllllllllll}
\hline 14 & 13 & 12 & 11 & 10 & 9 & 8 & 7 & 6 & 5 & 4 & 3 & 2 & 1
\end{tabular}

Figure S6. ${ }^{1} \mathrm{H}$ NMR spectrum of 3 in $\mathrm{CD}_{2} \mathrm{Cl}_{2}$ at $298 \mathrm{~K}$
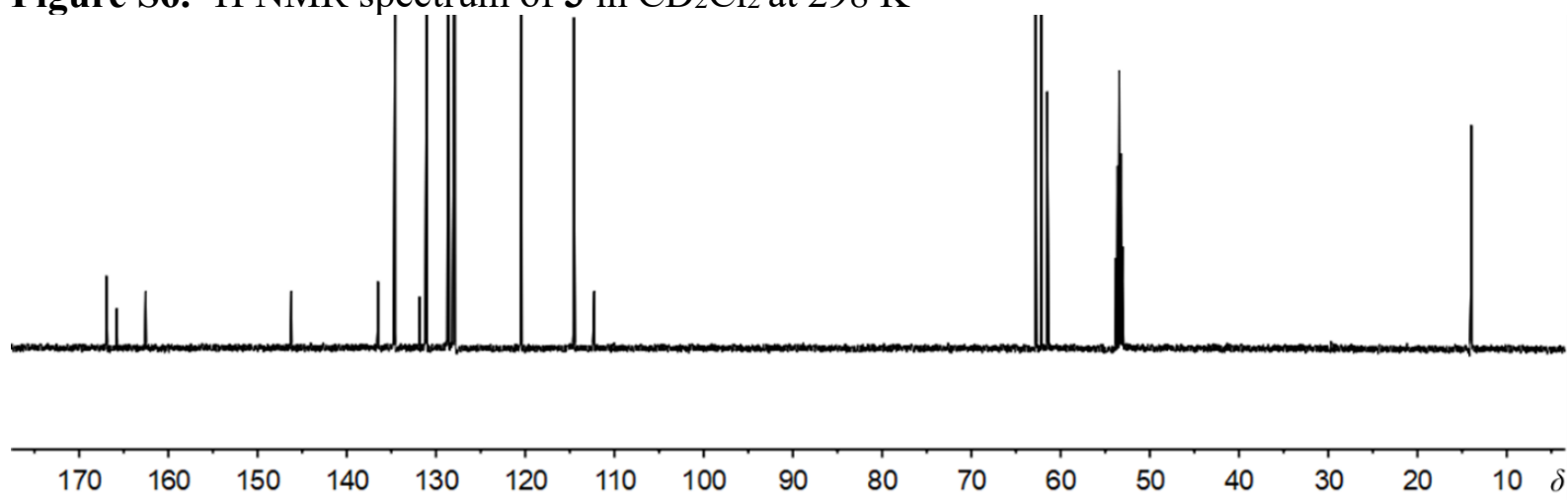

Figure S7. ${ }^{13} \mathrm{C}$ NMR spectrum of 3 in $\mathrm{CD}_{2} \mathrm{Cl}_{2}$ at $298 \mathrm{~K}$

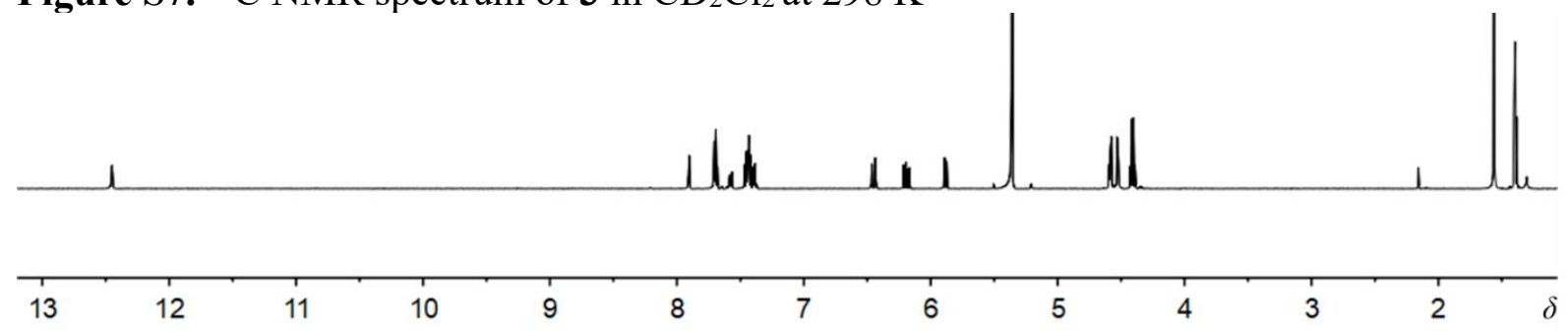

Figure S8. ${ }^{1} \mathrm{H}$ NMR spectrum of 4 in $\mathrm{CD}_{2} \mathrm{Cl}_{2}$ at $298 \mathrm{~K}$ 

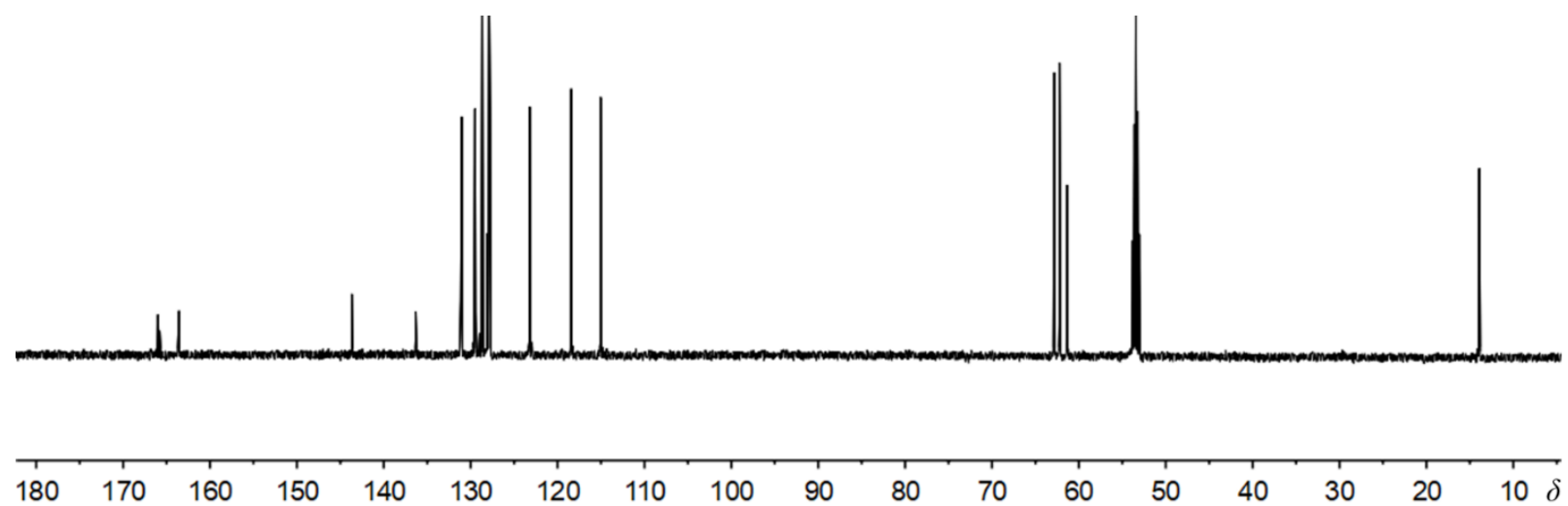

Figure S9. ${ }^{13} \mathrm{C}$ NMR spectrum of 4 in $\mathrm{CD}_{2} \mathrm{Cl}_{2}$ at $298 \mathrm{~K}$
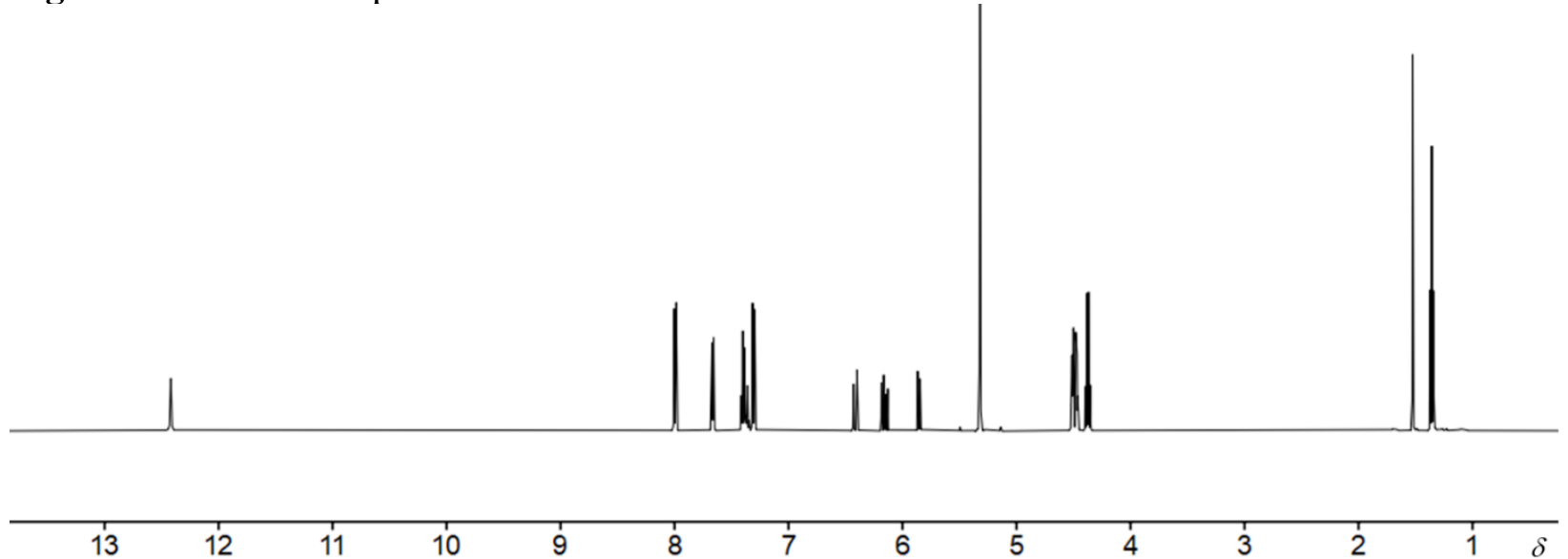

Figure S10. ${ }^{1} \mathrm{H}$ NMR spectrum of 5 in $\mathrm{CD}_{2} \mathrm{Cl}_{2}$ at $298 \mathrm{~K}$

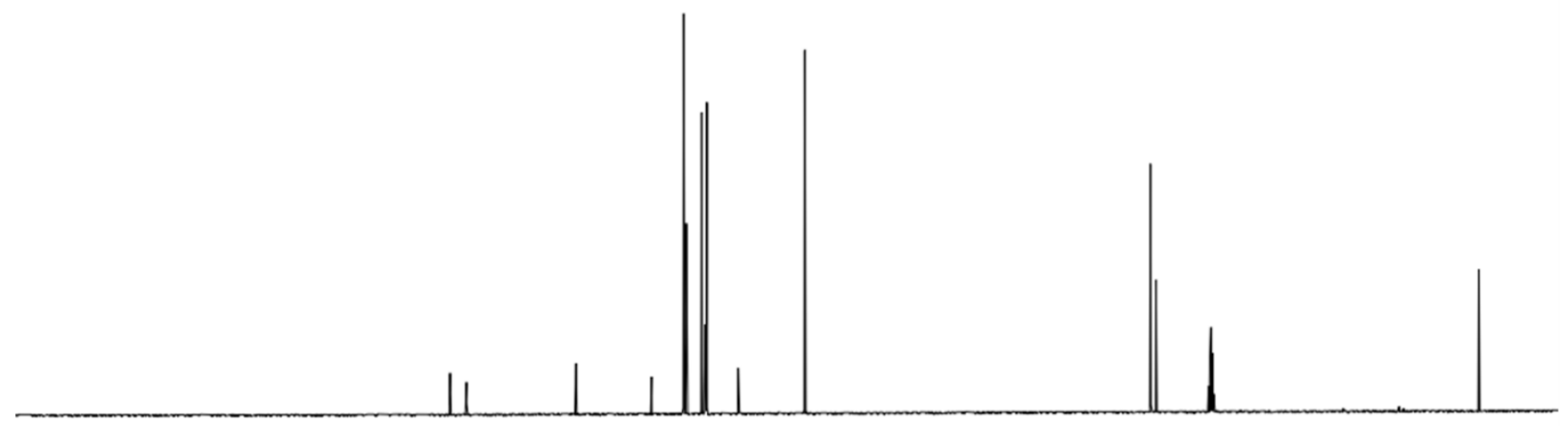

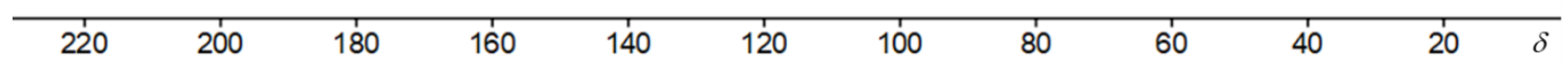

Figure S11. ${ }^{13} \mathrm{C}$ NMR spectrum of 5 in $\mathrm{CD}_{2} \mathrm{Cl}_{2}$ at $298 \mathrm{~K}$ 


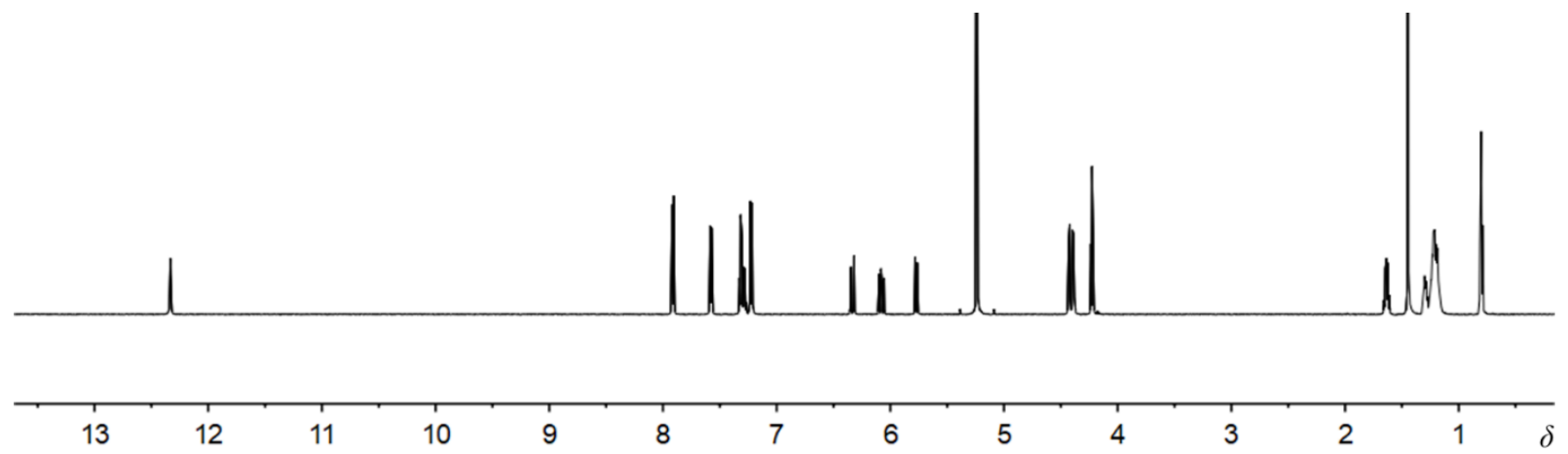

Figure S12. ${ }^{1} \mathrm{H}$ NMR spectrum of 6 in $\mathrm{CD}_{2} \mathrm{Cl}_{2}$ at $298 \mathrm{~K}$
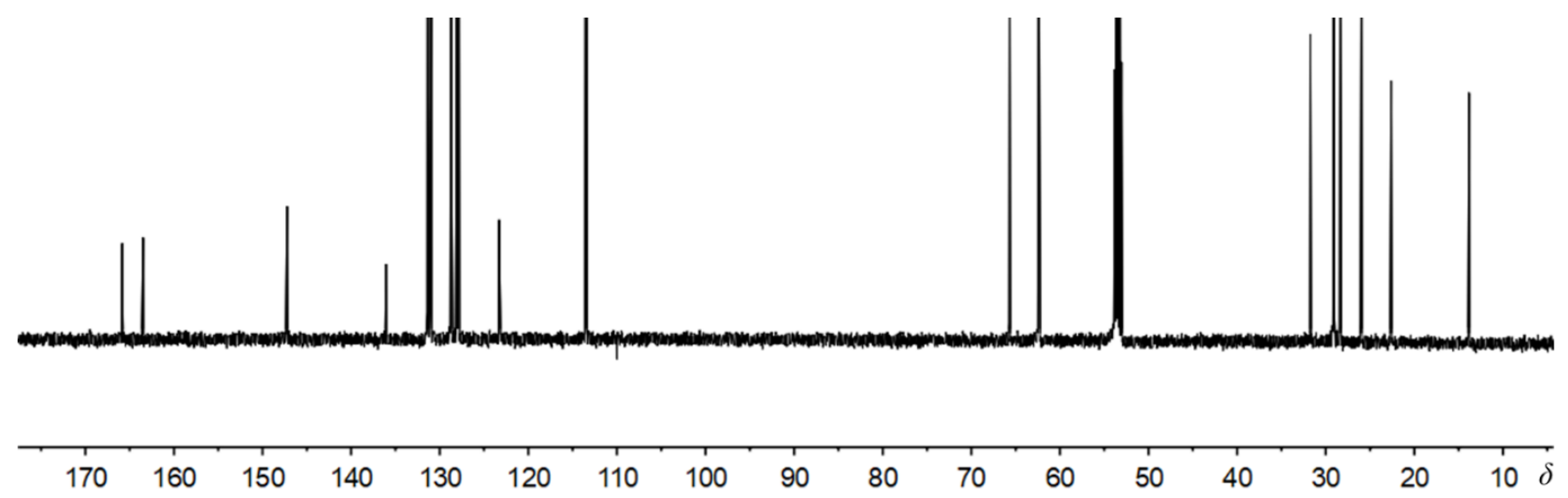

Figure S13. ${ }^{13} \mathrm{C}$ NMR spectrum of 6 in $\mathrm{CD}_{2} \mathrm{Cl}_{2}$ at $298 \mathrm{~K}$
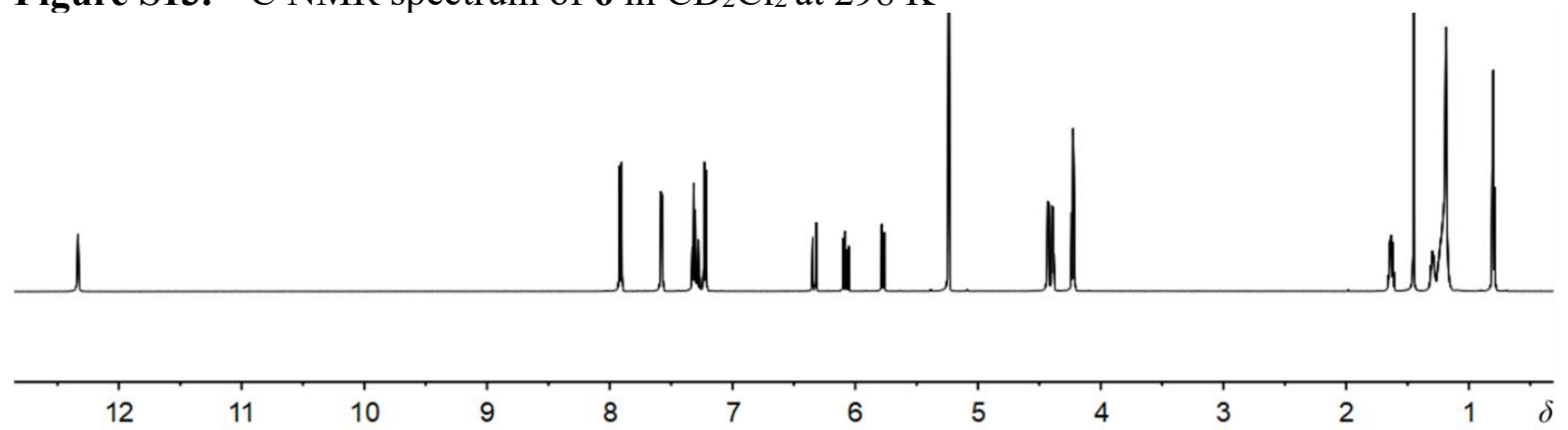

Figure S14. ${ }^{1} \mathrm{H}$ NMR spectrum of 7 in $\mathrm{CD}_{2} \mathrm{Cl}_{2}$ at $298 \mathrm{~K}$
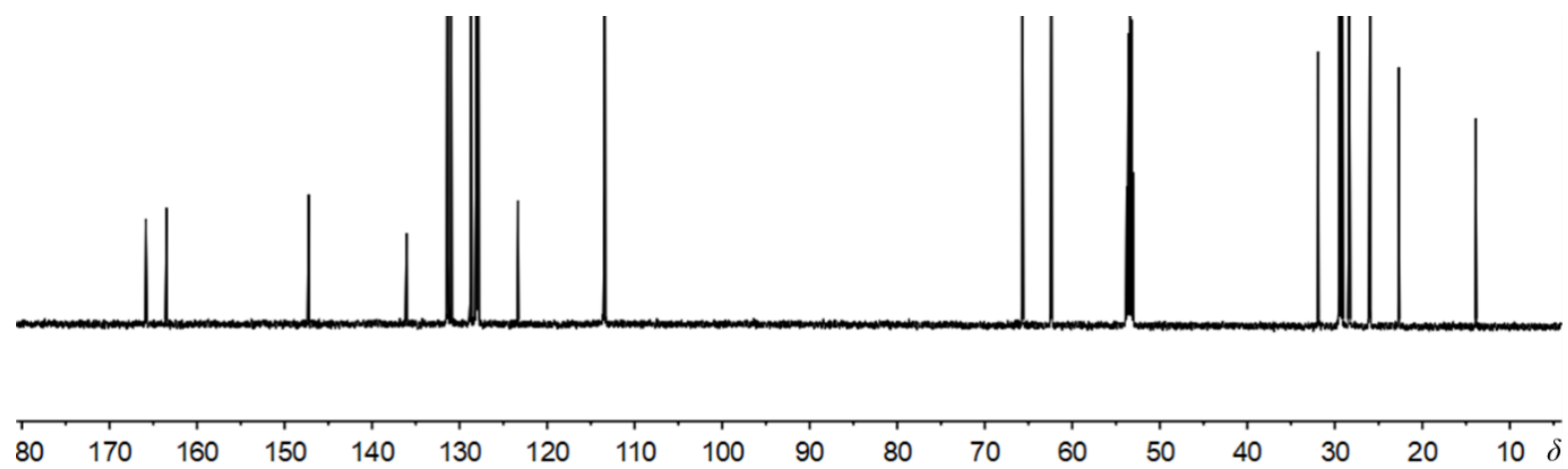

Figure S15. ${ }^{13} \mathrm{C}$ NMR spectrum of 7 in $\mathrm{CD}_{2} \mathrm{Cl}_{2}$ at $298 \mathrm{~K}$ 


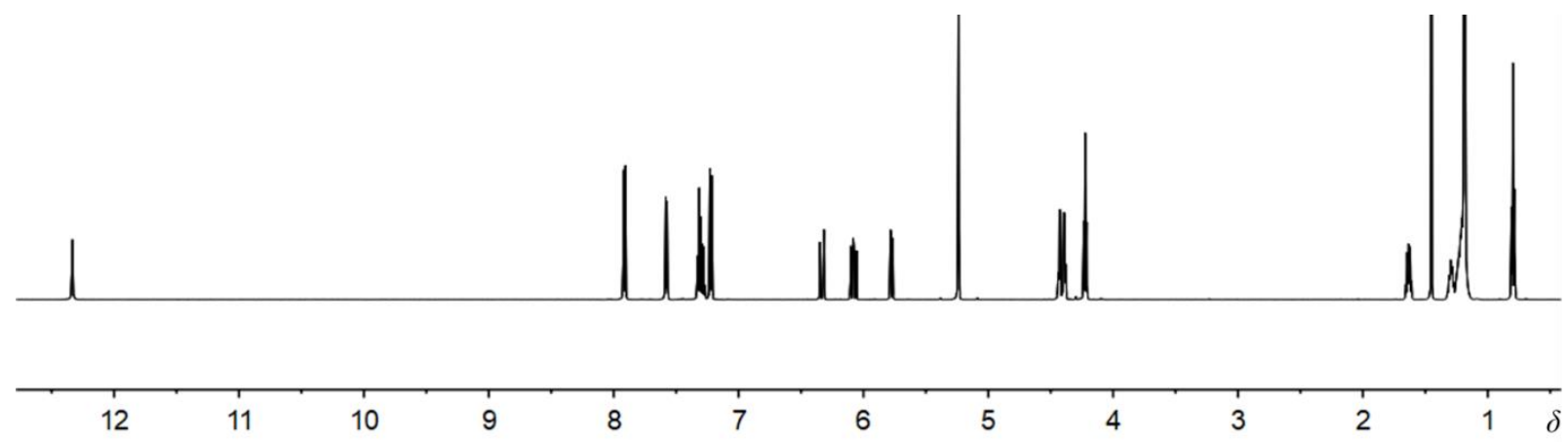

Figure S16. ${ }^{1} \mathrm{H}$ NMR spectrum of 8 in $\mathrm{CD}_{2} \mathrm{Cl}_{2}$ at $298 \mathrm{~K}$
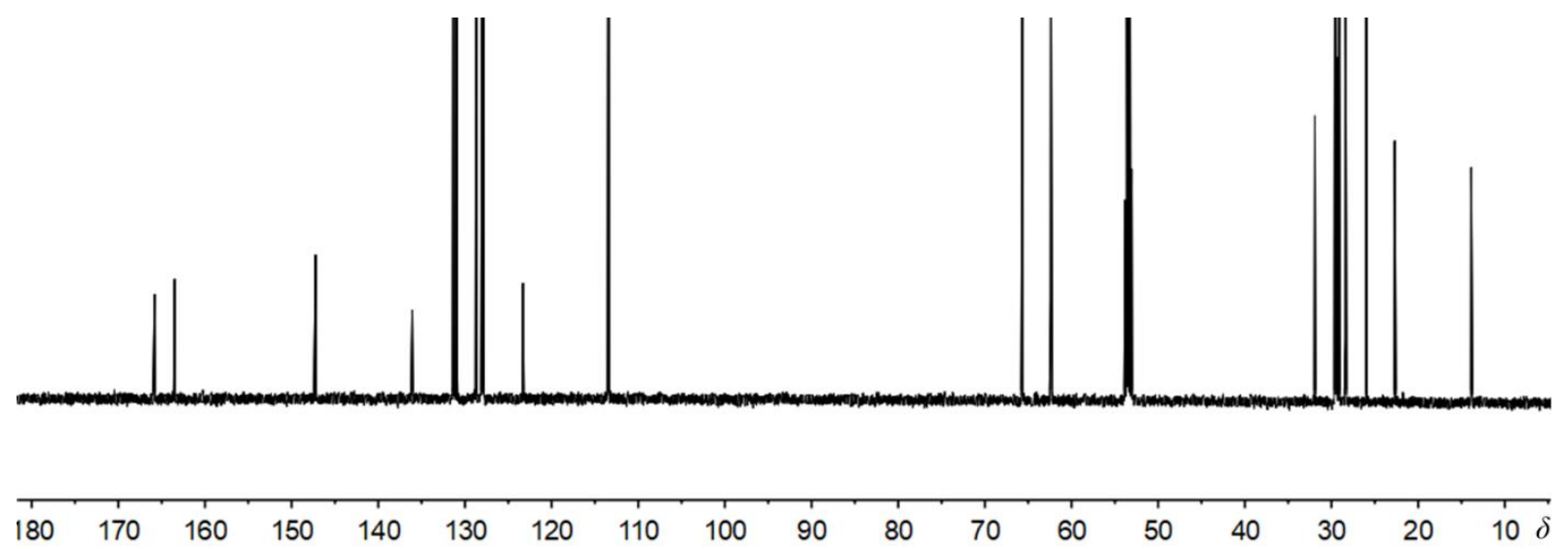

Figure S17. ${ }^{13} \mathrm{C}$ NMR spectrum of 8 in $\mathrm{CD}_{2} \mathrm{Cl}_{2}$ at $298 \mathrm{~K}$

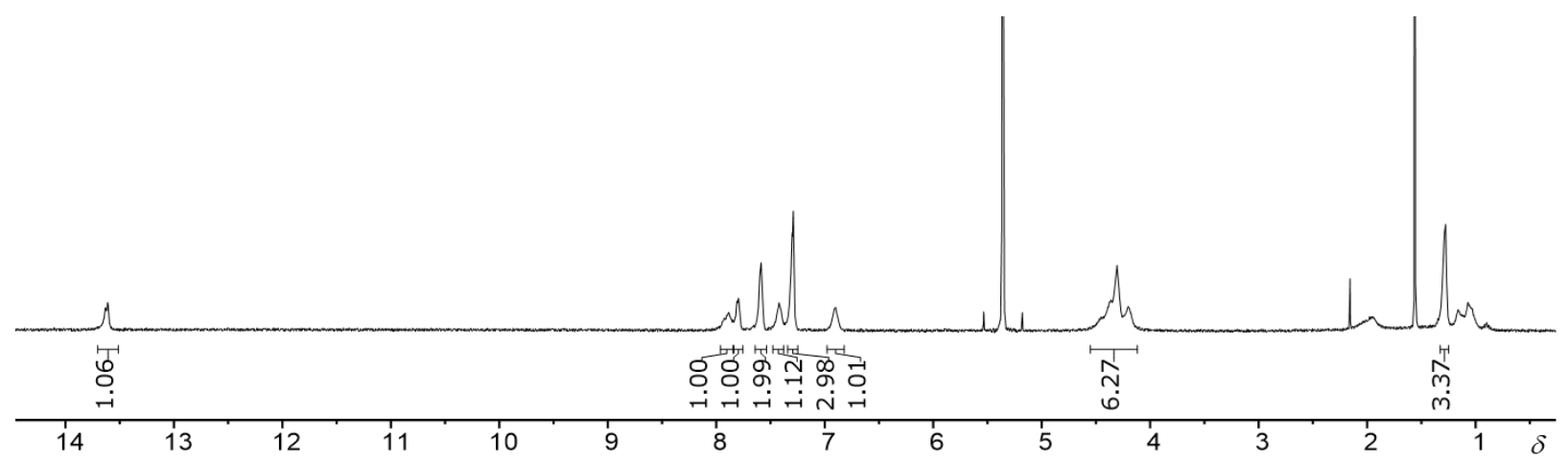

Figure S18. ${ }^{1} \mathrm{H}$ NMR spectrum of $\mathbf{P 1}$ in $\mathrm{CD}_{2} \mathrm{Cl}_{2}$ at $298 \mathrm{~K}$ 


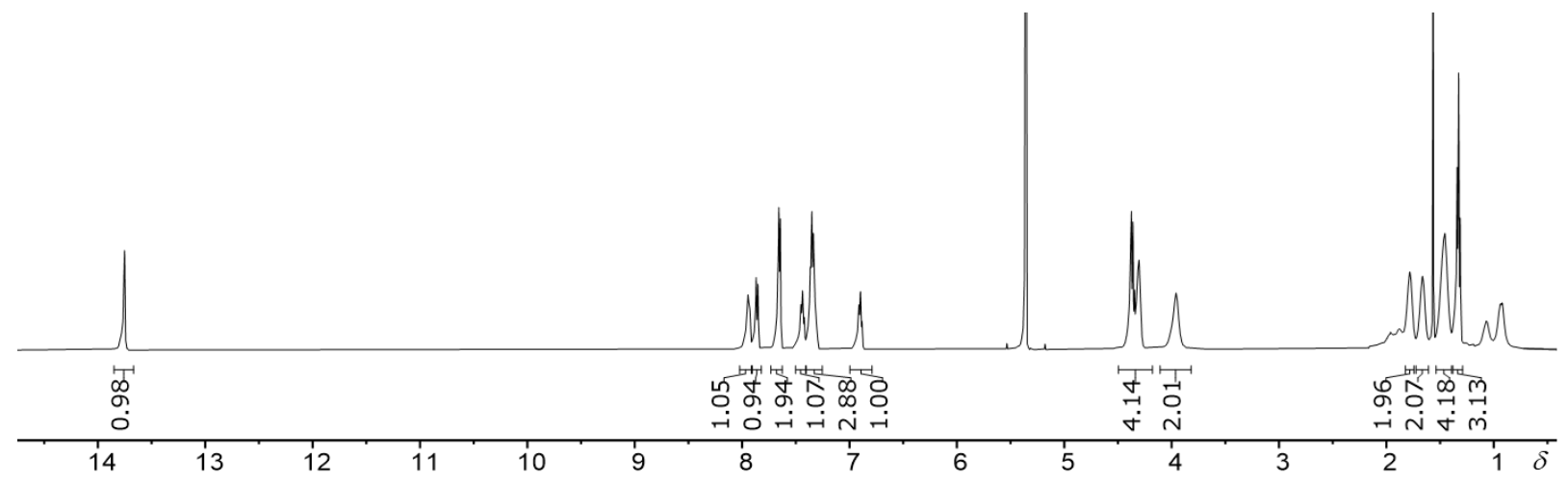

Figure S19. ${ }^{1} \mathrm{H} \mathrm{NMR}$ spectrum of $\mathbf{P 2}$ in $\mathrm{CD}_{2} \mathrm{Cl}_{2}$ at $298 \mathrm{~K}$

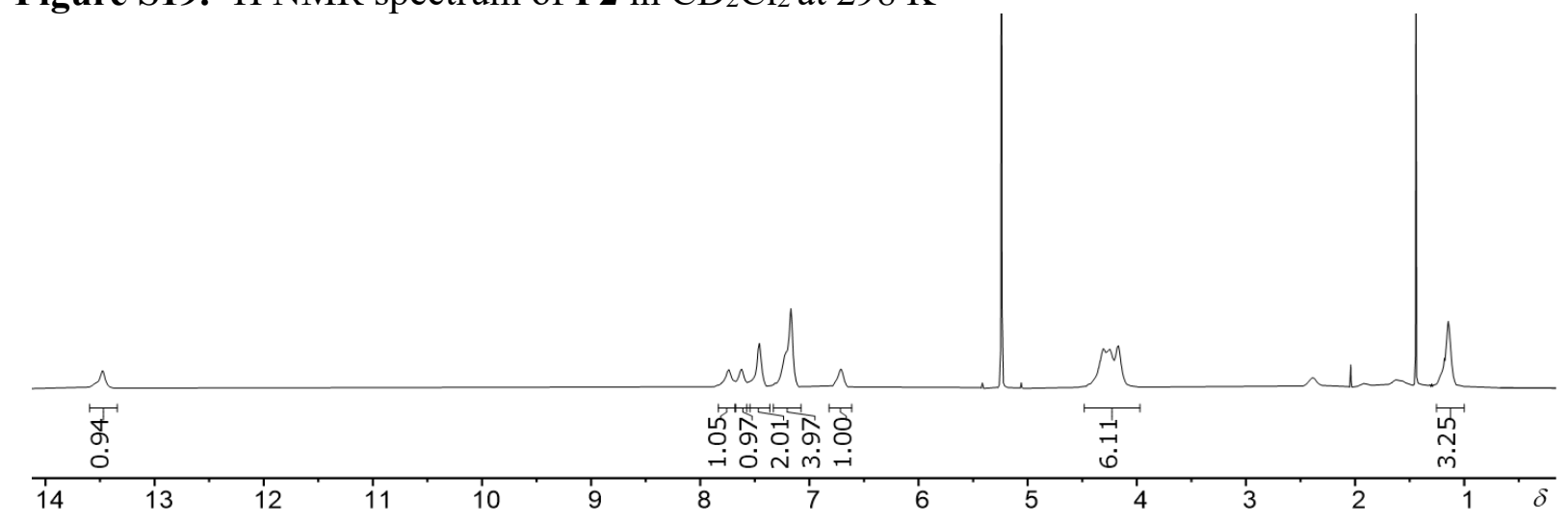

Figure S20. ${ }^{1} \mathrm{H} \mathrm{NMR}$ spectrum of $\mathbf{P 3}$ in $\mathrm{CD}_{2} \mathrm{Cl}_{2}$ at $298 \mathrm{~K}$

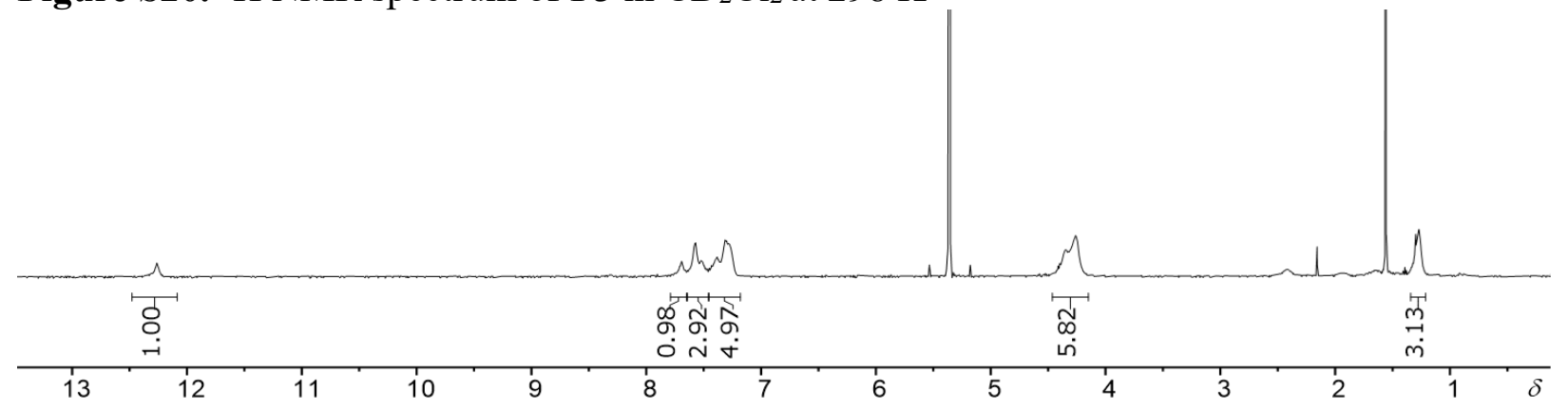

Figure S21. ${ }^{1} \mathrm{H}$ NMR spectrum of $\mathbf{P 4}$ in $\mathrm{CD}_{2} \mathrm{Cl}_{2}$ at $298 \mathrm{~K}$

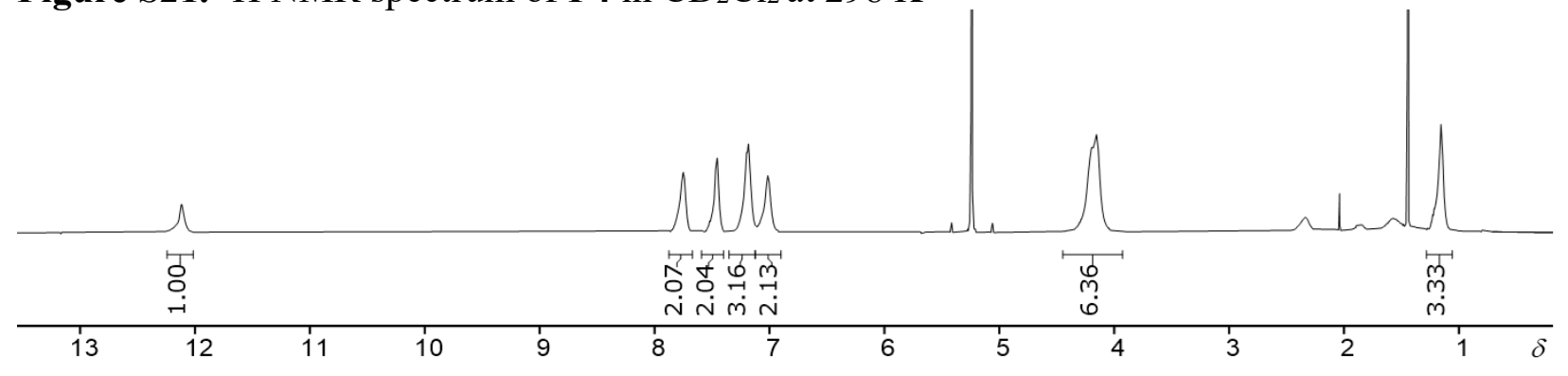

Figure S22. ${ }^{1} \mathrm{H} \mathrm{NMR}$ spectrum of $\mathbf{P 5}$ in $\mathrm{CD}_{2} \mathrm{Cl}_{2}$ at $298 \mathrm{~K}$ 


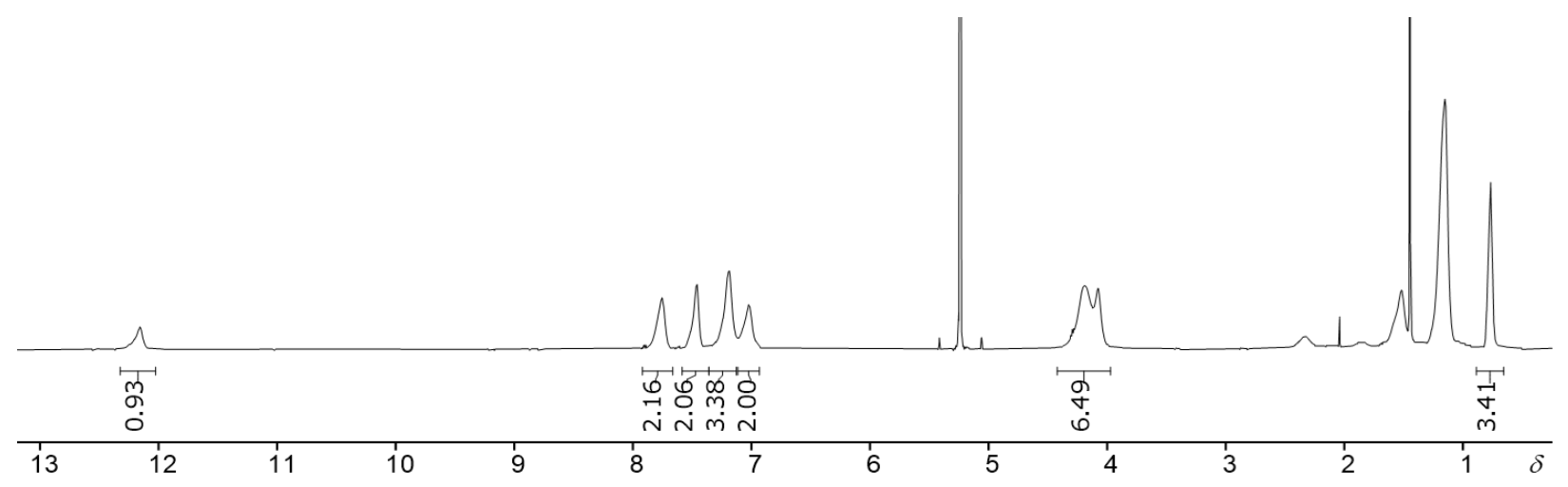

Figure S23. ${ }^{1} \mathrm{H}$ NMR spectrum of $\mathbf{P 6}$ in $\mathrm{CD}_{2} \mathrm{Cl}_{2}$ at $298 \mathrm{~K}$

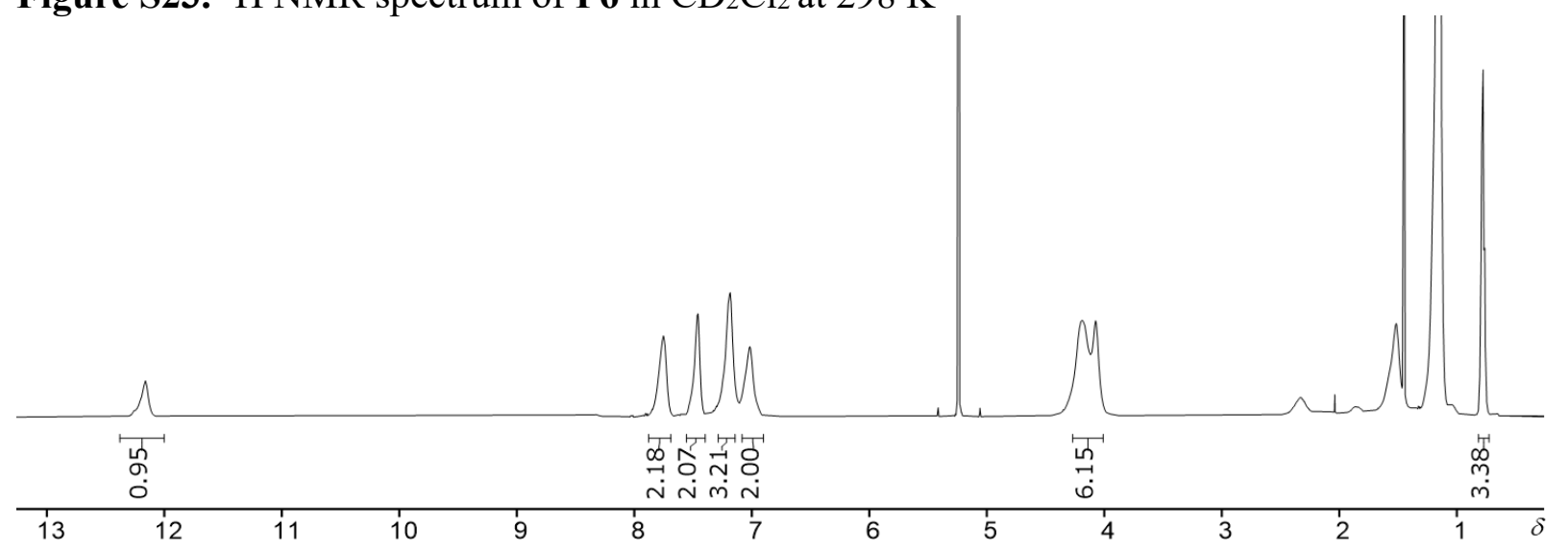

Figure S24. ${ }^{1} \mathrm{H}$ NMR spectrum of $\mathbf{P 7}$ in $\mathrm{CD}_{2} \mathrm{Cl}_{2}$ at $298 \mathrm{~K}$

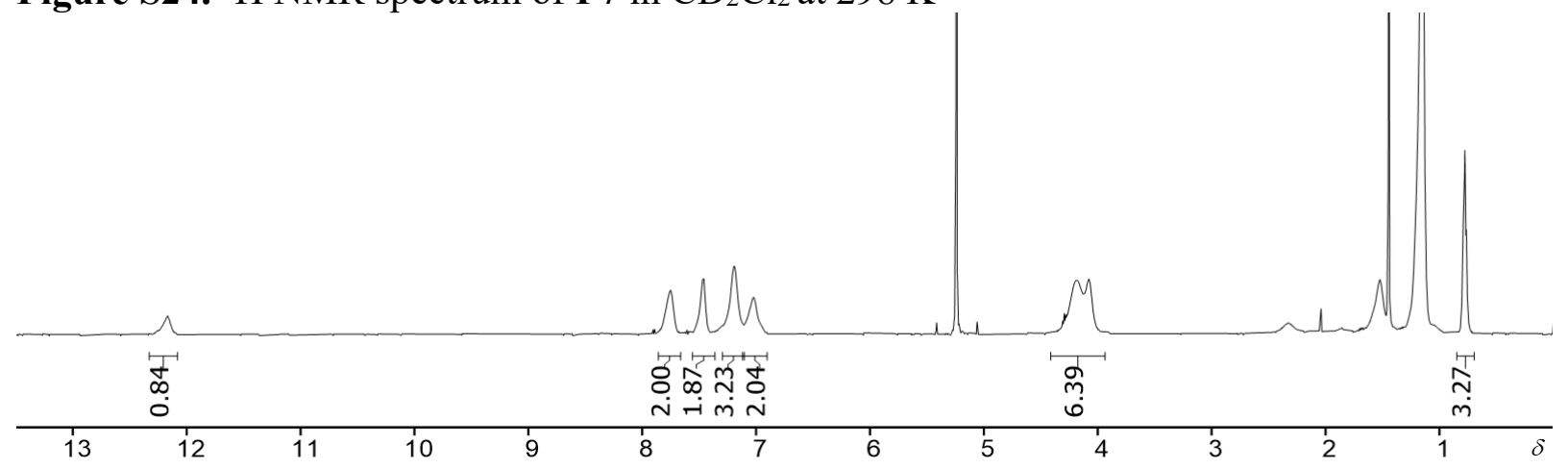

Figure S25. ${ }^{1} \mathrm{H}$ NMR spectrum of $\mathbf{P 8}$ in $\mathrm{CD}_{2} \mathrm{Cl}_{2}$ at $298 \mathrm{~K}$

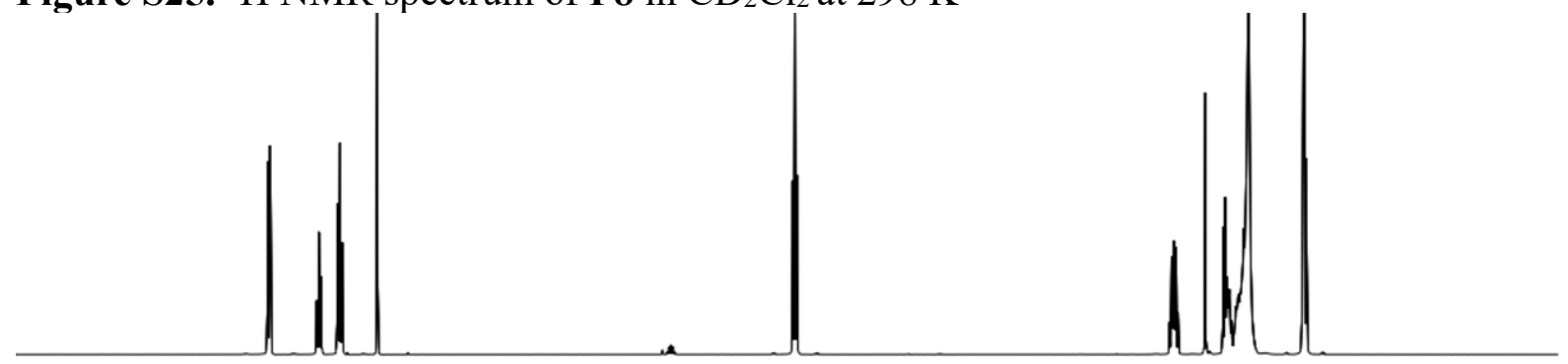

\begin{tabular}{lllllllllll}
\hline 9 & 8 & 7 & 6 & 5 & 4 & 3 & 1 & 1 & 0 & $\delta$
\end{tabular}


Figure S26. ${ }^{1} \mathrm{H}$ NMR spectrum of $\mathbf{1 1}$ in $\mathrm{CDCl}_{3}$ at $298 \mathrm{~K}$

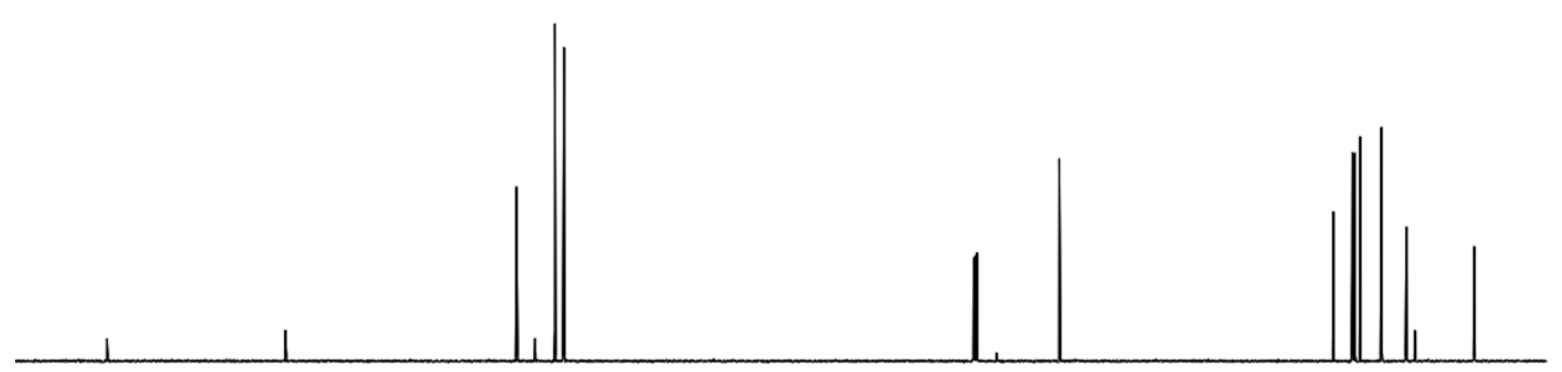

$180 \quad 160$

140

120

100

80

60

40

Figure S27. ${ }^{13} \mathrm{C}$ NMR spectrum of 11 in $\mathrm{CDCl}_{3}$ at $298 \mathrm{~K}$

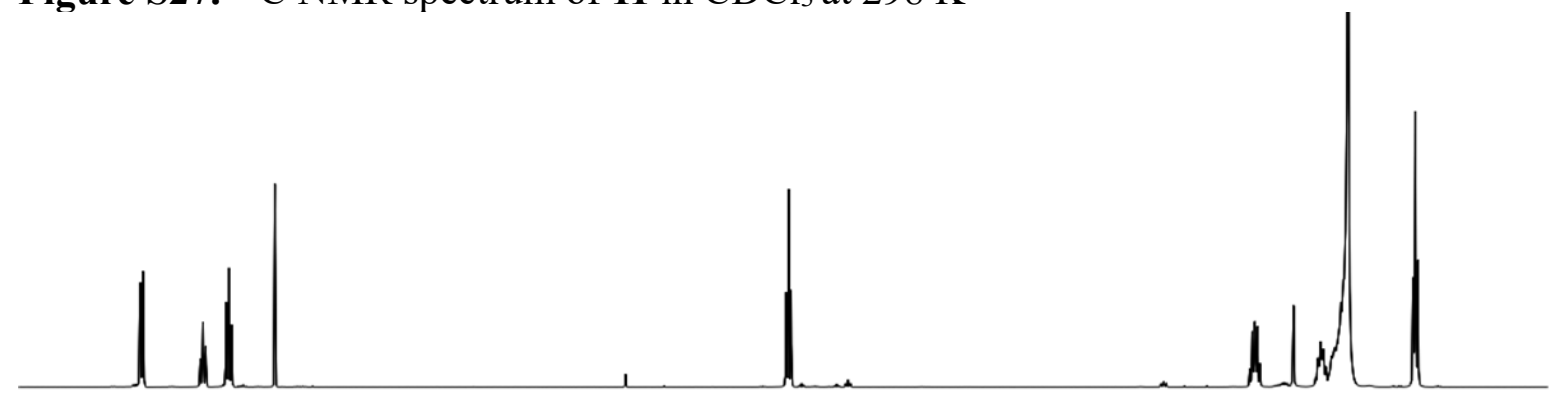

\begin{tabular}{llllllllll}
\hline 8 & 7 & 1 & 6 & 5 & 4 & 3 & 2 & 1 & $\delta$ \\
\hline
\end{tabular}

Figure S28. ${ }^{1} \mathrm{H}$ NMR spectrum of 12 in $\mathrm{CDCl}_{3}$ at $298 \mathrm{~K}$

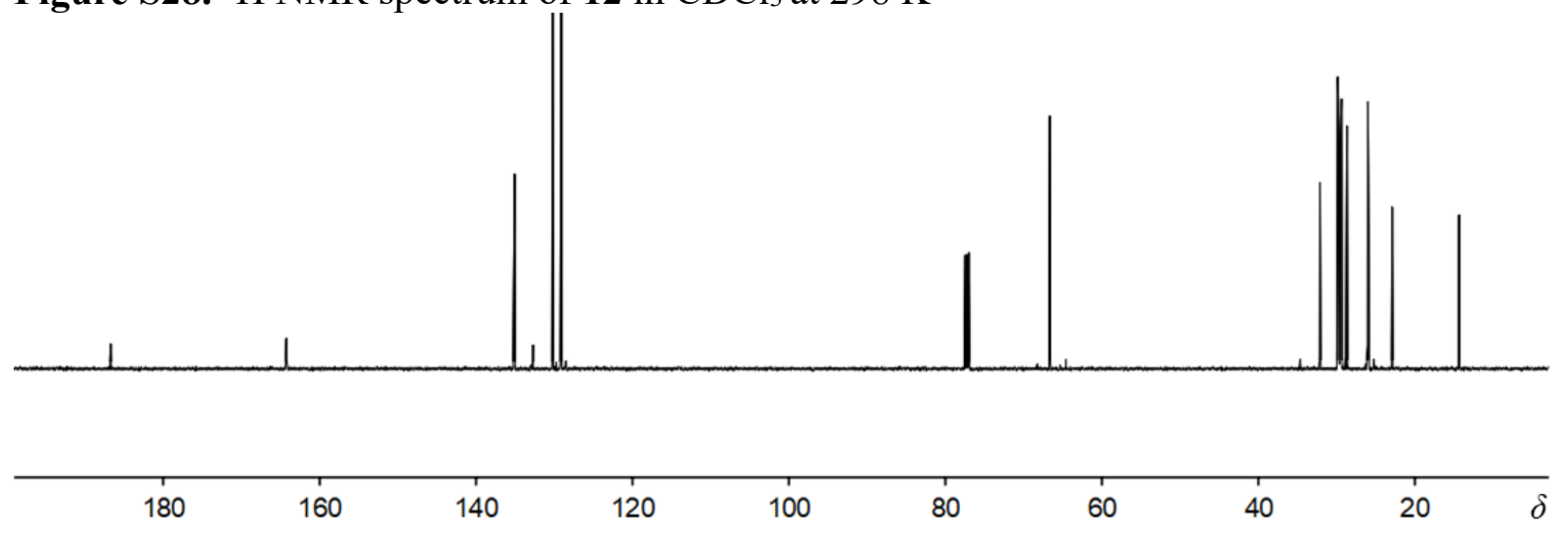

Figure S29. ${ }^{13} \mathrm{C}$ NMR spectrum of 12 in $\mathrm{CDCl}_{3}$ at $298 \mathrm{~K}$ 


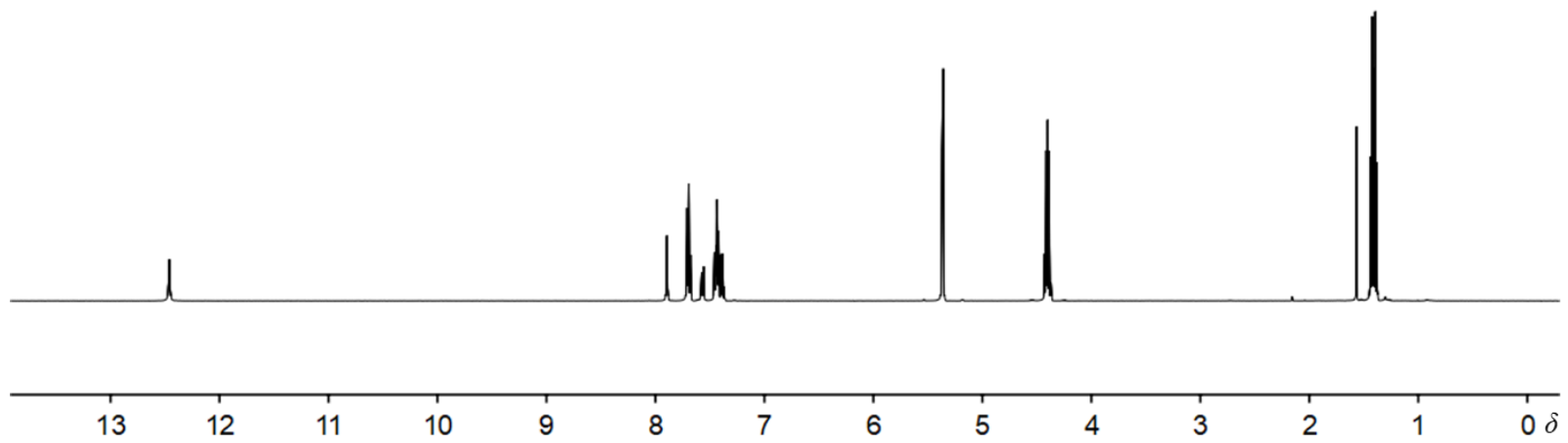

Figure S30. ${ }^{1} \mathrm{H}$ NMR spectrum of 14 in $\mathrm{CD}_{2} \mathrm{Cl}_{2}$ at $298 \mathrm{~K}$

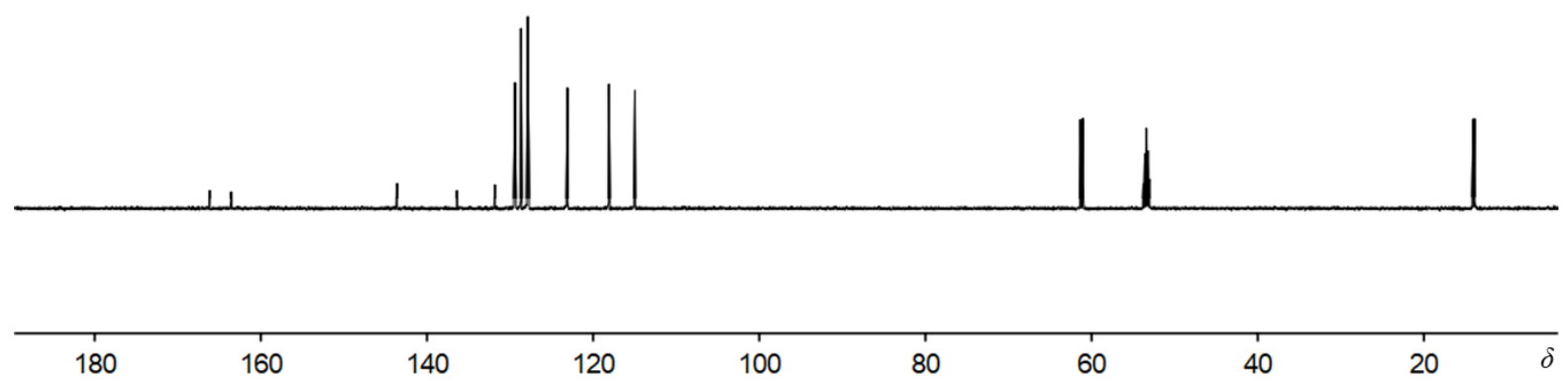

Figure S31. ${ }^{13} \mathrm{C}$ NMR spectrum of 14 in $\mathrm{CD}_{2} \mathrm{Cl}_{2}$ at $298 \mathrm{~K}$

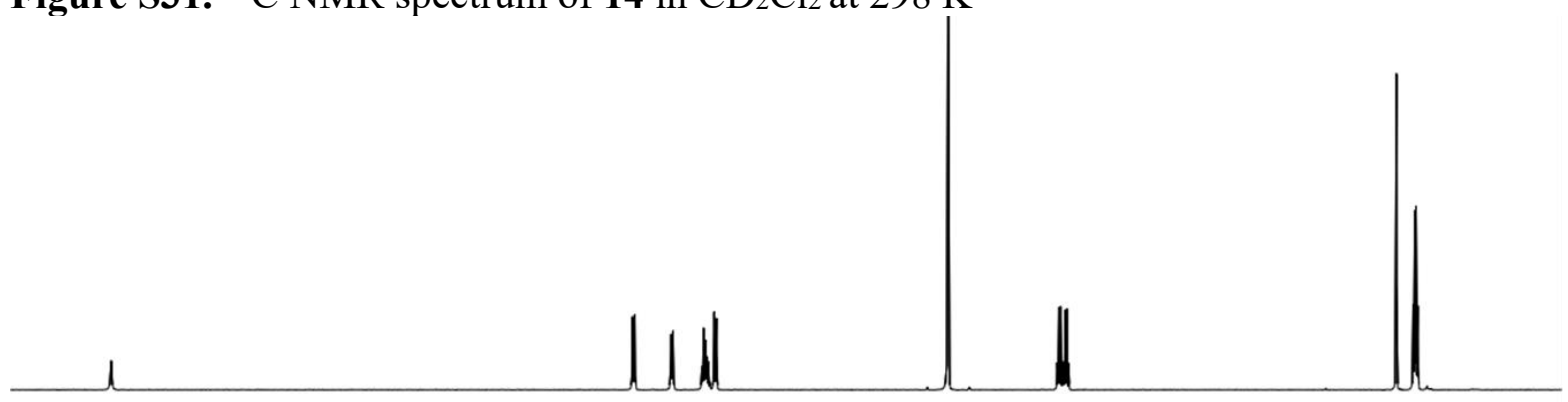

\begin{tabular}{|c|c|}
\hline 13 & \\
\hline
\end{tabular}

Figure S32. ${ }^{1} \mathrm{H}$ NMR spectrum of 15 in $\mathrm{CD}_{2} \mathrm{Cl}_{2}$ at $298 \mathrm{~K}$

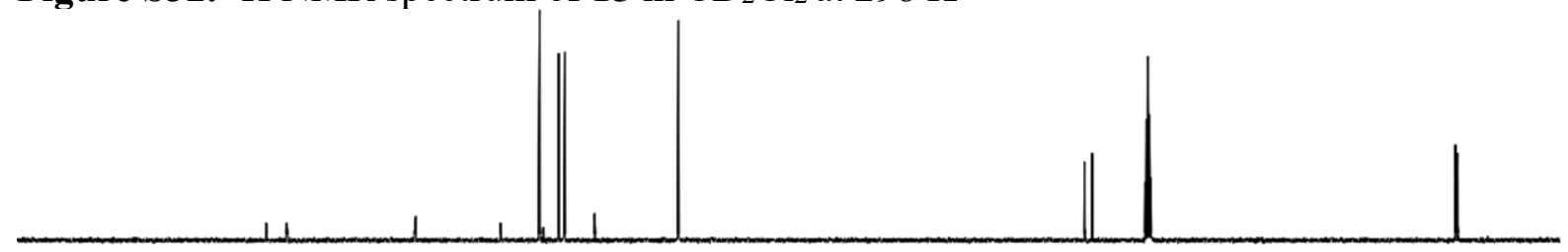

180

160

140

120

100

80

60

40

20

Figure S33. ${ }^{13} \mathrm{C}$ NMR spectrum of 15 in $\mathrm{CD}_{2} \mathrm{Cl}_{2}$ at $298 \mathrm{~K}$ 


\section{Photoswitching of the Monomers}

Spectrophotometric grade solvents were used for the absorption studies. Monomer solutions $\left(3.0 \mathrm{~mL}, 1.0 \times 10^{-5} \mathrm{M}\right)$ in DCM were prepared and transferred into $1.0 \mathrm{~cm}$ quartz cuvettes for immediate UV/Vis absorption measurements. The solutions were then irradiated, and corresponding UV spectra recorded.

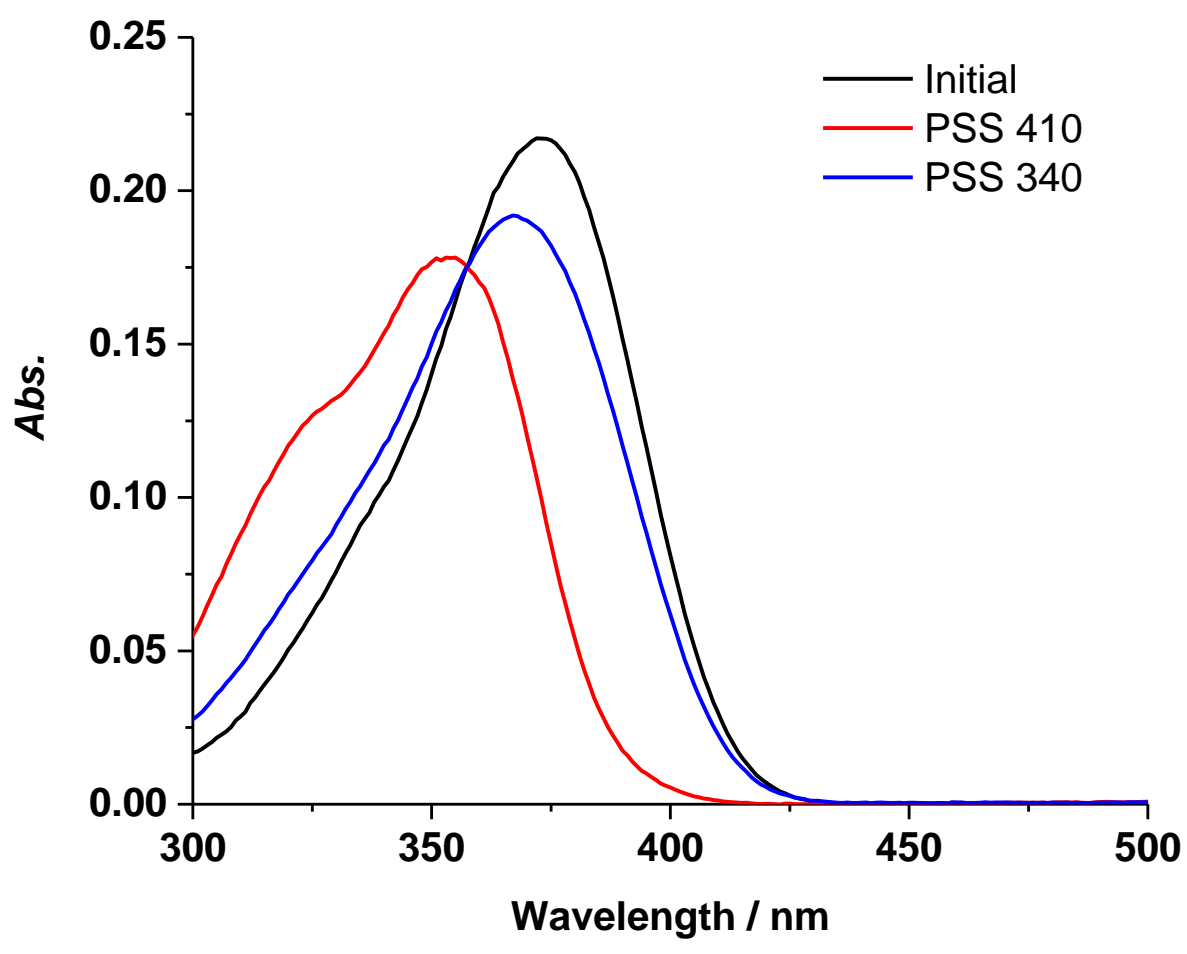

Figure S34. Light induced $Z / E$ isomerization of $1\left(1 \times 10^{-5} \mathrm{M}\right)$ in DCM 


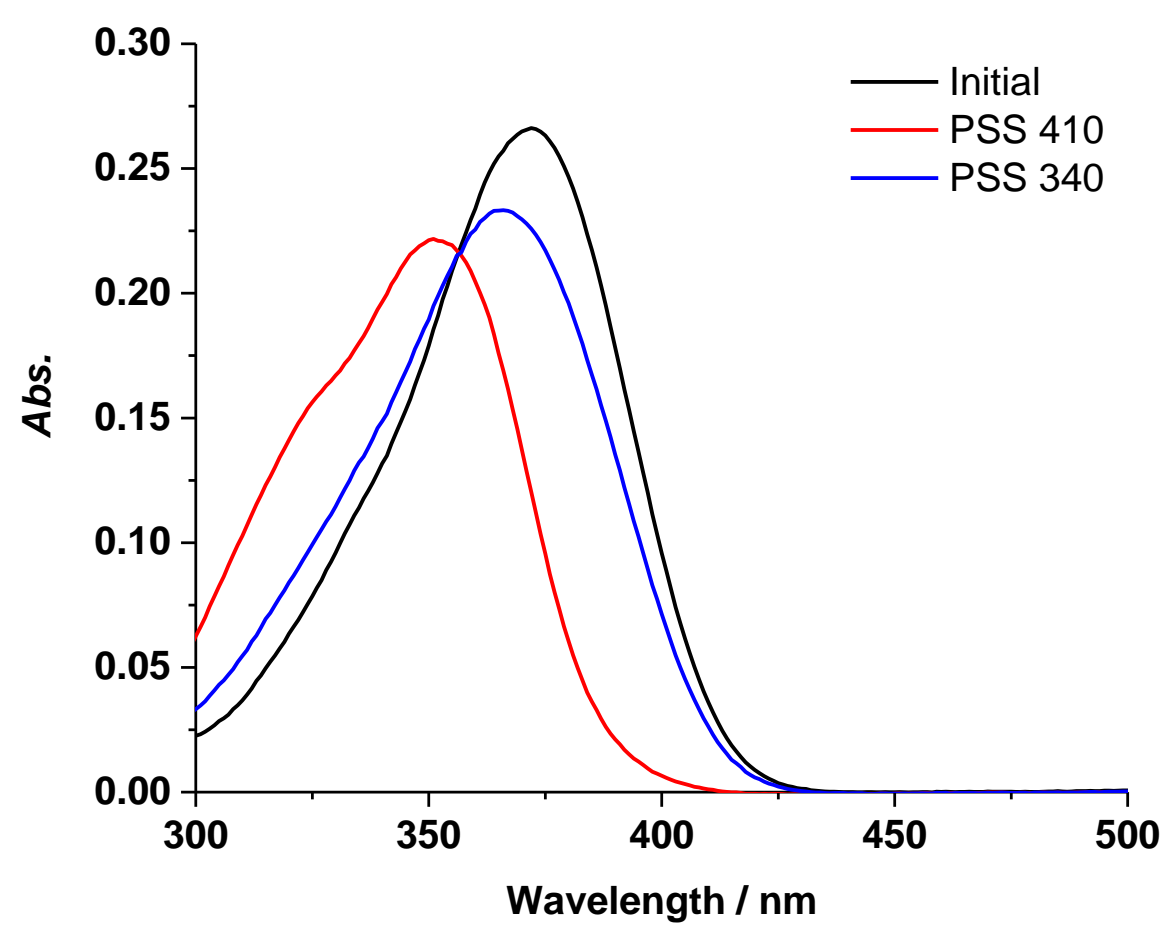

Figure S35. Light induced $Z / E$ isomerization of $2\left(1 \times 10^{-5} \mathrm{M}\right)$ in DCM

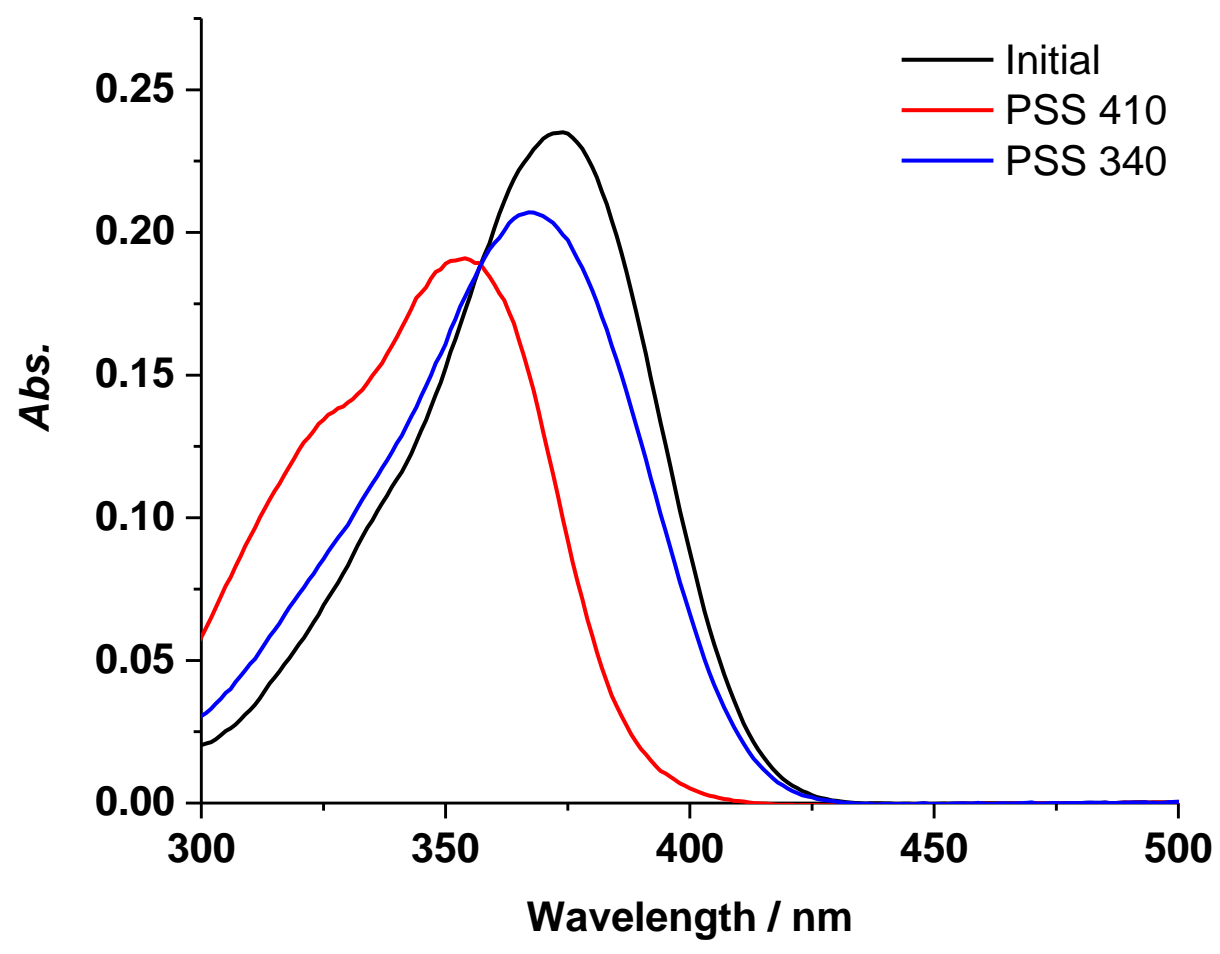

Figure S36. Light induced $Z / E$ isomerization of $\mathbf{3}\left(1 \times 10^{-5} \mathrm{M}\right)$ in DCM 


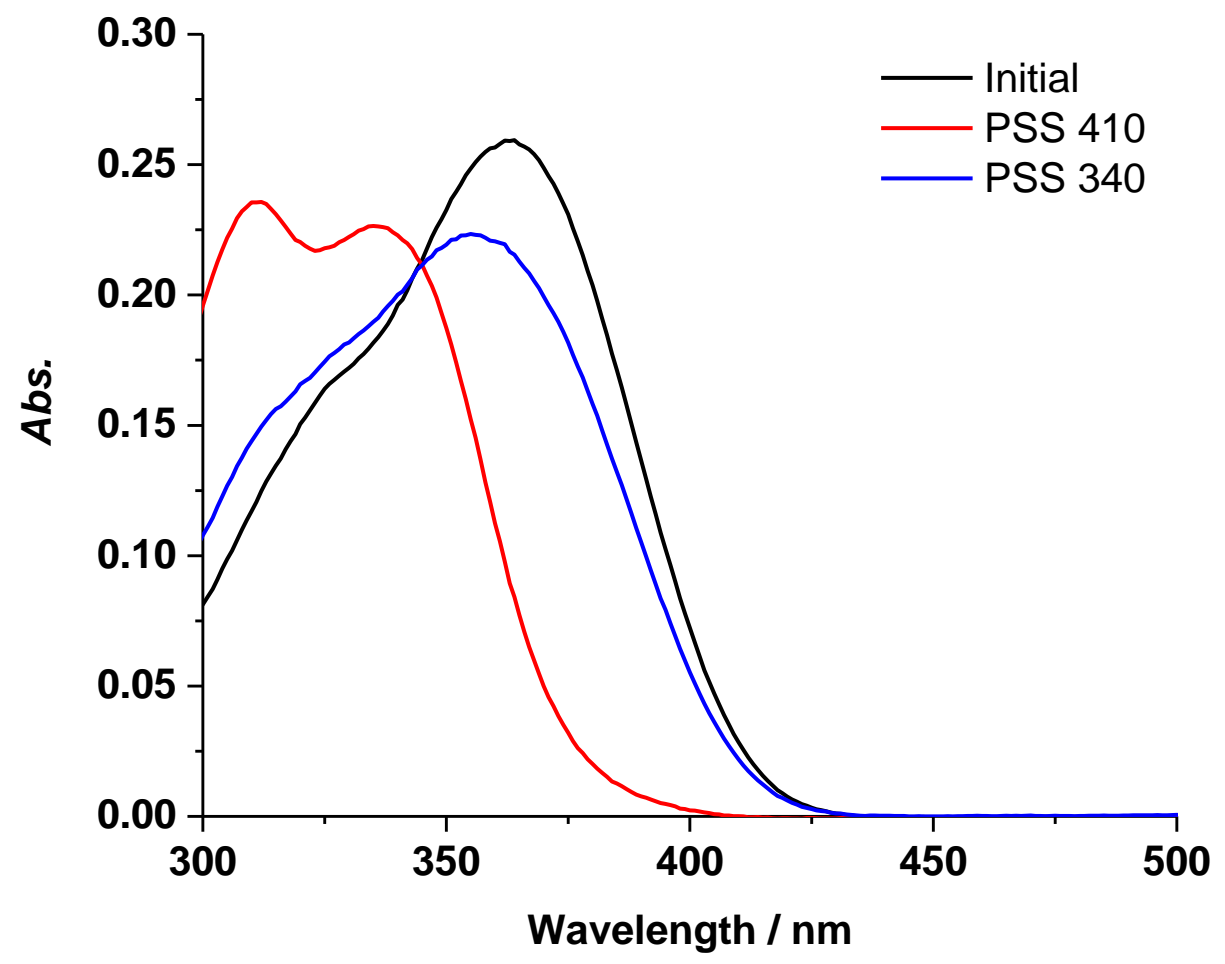

Figure S37. Light induced $Z / E$ isomerization of $4\left(1 \times 10^{-5} \mathrm{M}\right)$ in DCM

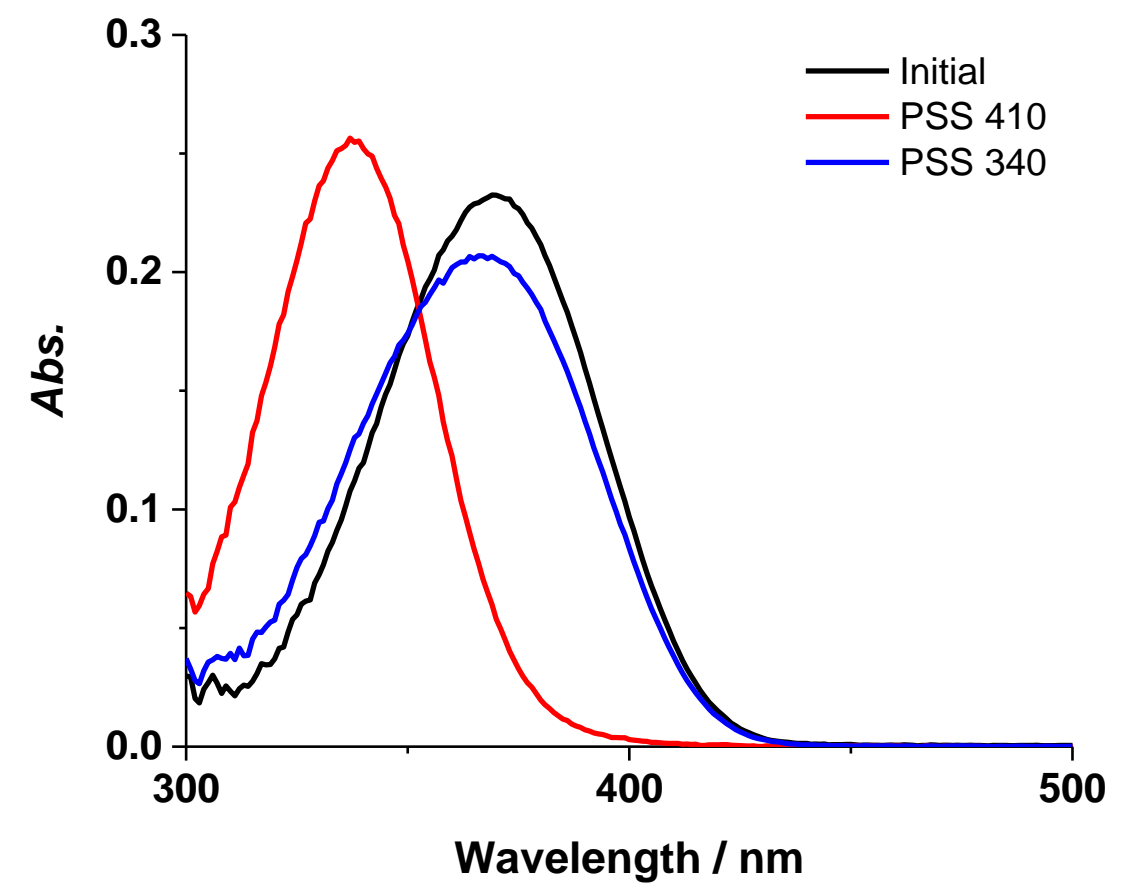

Figure S38. Light induced $Z / E$ isomerization of $5\left(1 \times 10^{-5} \mathrm{M}\right)$ in DCM 


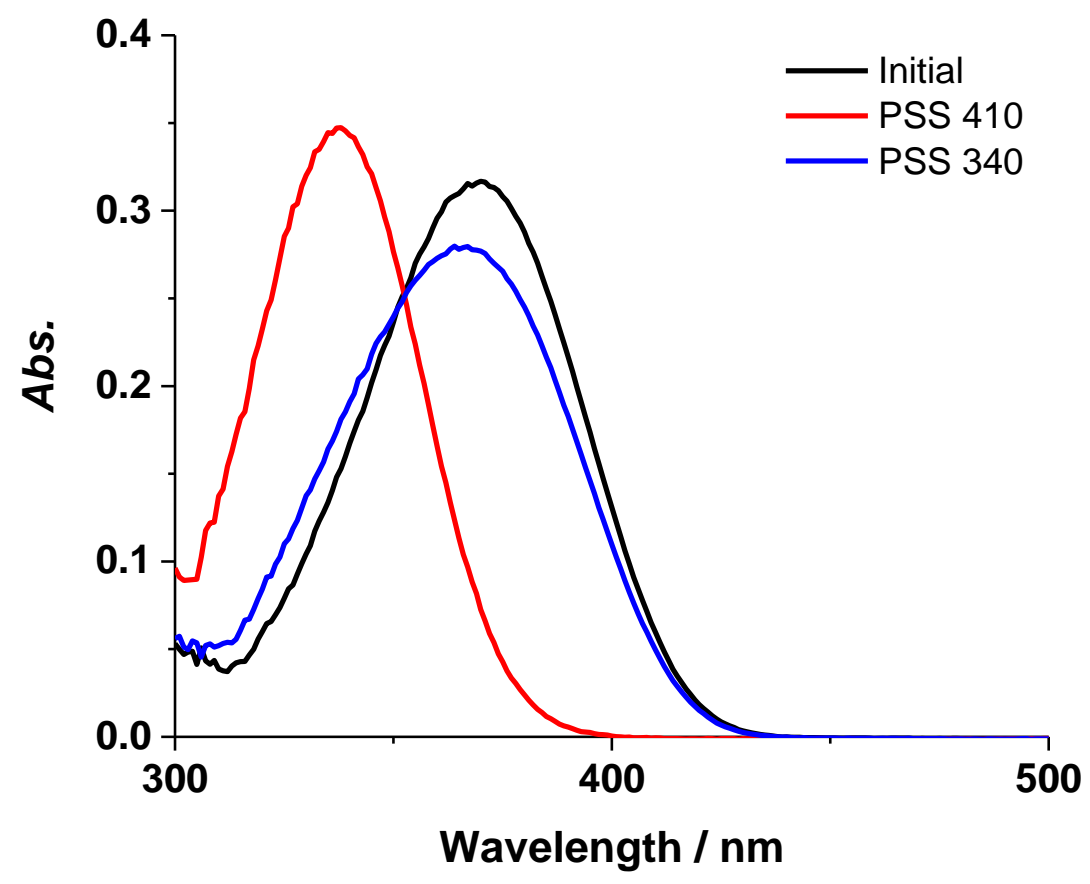

Figure S39. Light induced $Z / E$ isomerization of $6\left(1 \times 10^{-5} \mathrm{M}\right)$ in DCM

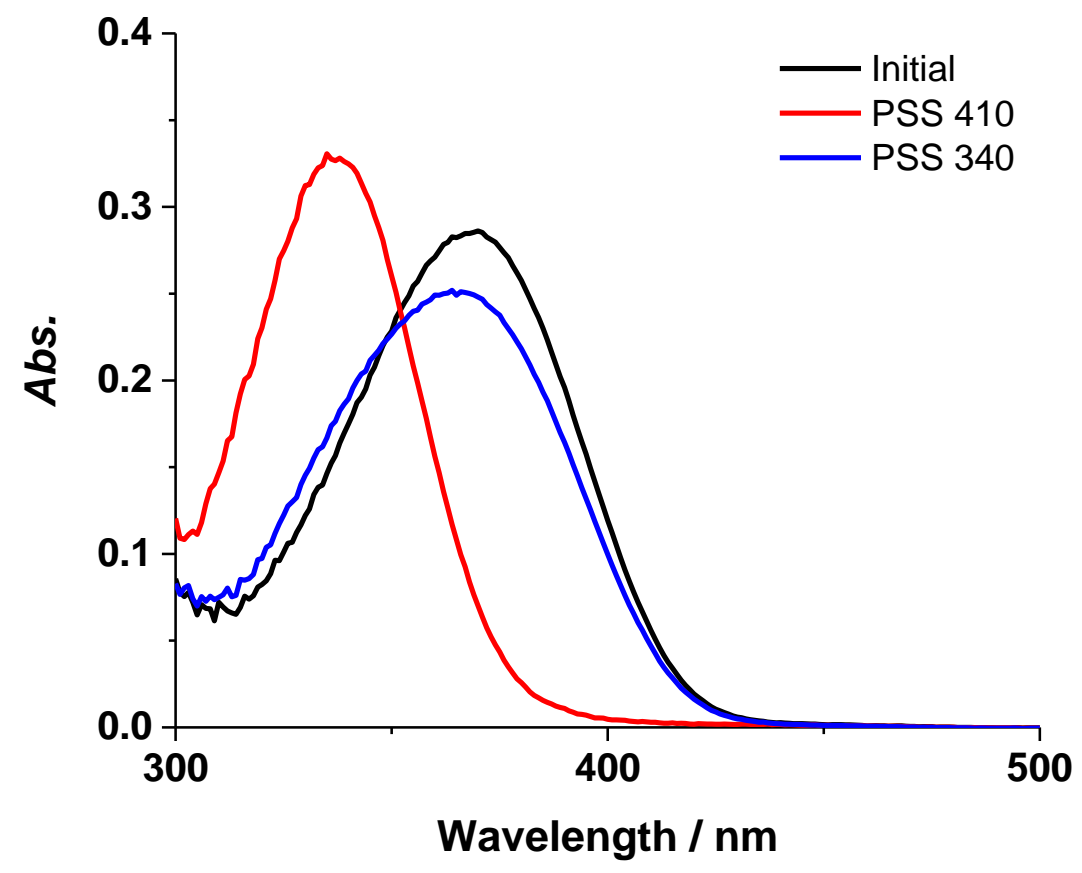

Figure S40. Light induced $Z / E$ isomerization of $7\left(1 \times 10^{-5} \mathrm{M}\right)$ in DCM 


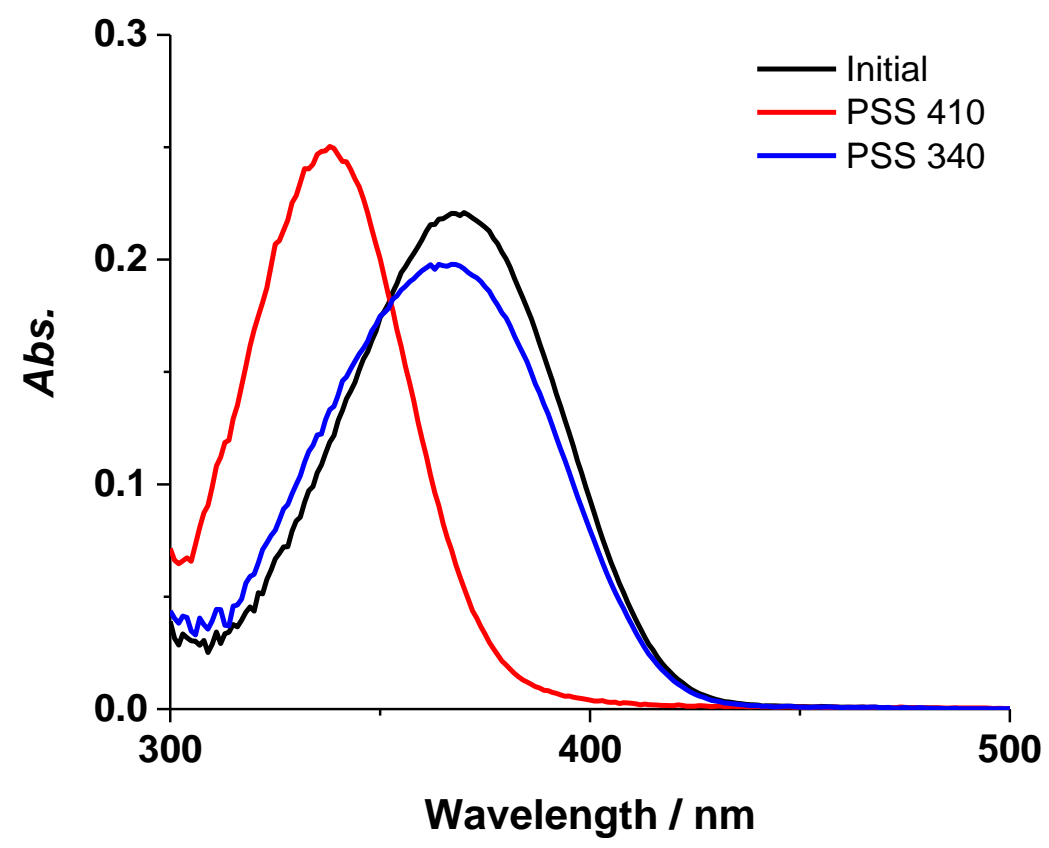

Figure S41. Light induced $Z / E$ isomerization of $8\left(1 \times 10^{-5} \mathrm{M}\right)$ in DCM 


\section{Photoswitching of the Polymers in solution}

Spectrophotometric grade solvents were used for the absorption studies. Polymer solutions (3.0 $\mathrm{mL}, 4 \mu \mathrm{g} / \mathrm{mL}$ ) in DCM were prepared and transferred into $1.0 \mathrm{~cm}$ quartz cuvettes for immediate UV/Vis absorption measurements. The solutions were then irradiated, and corresponding UV spectra recorded.

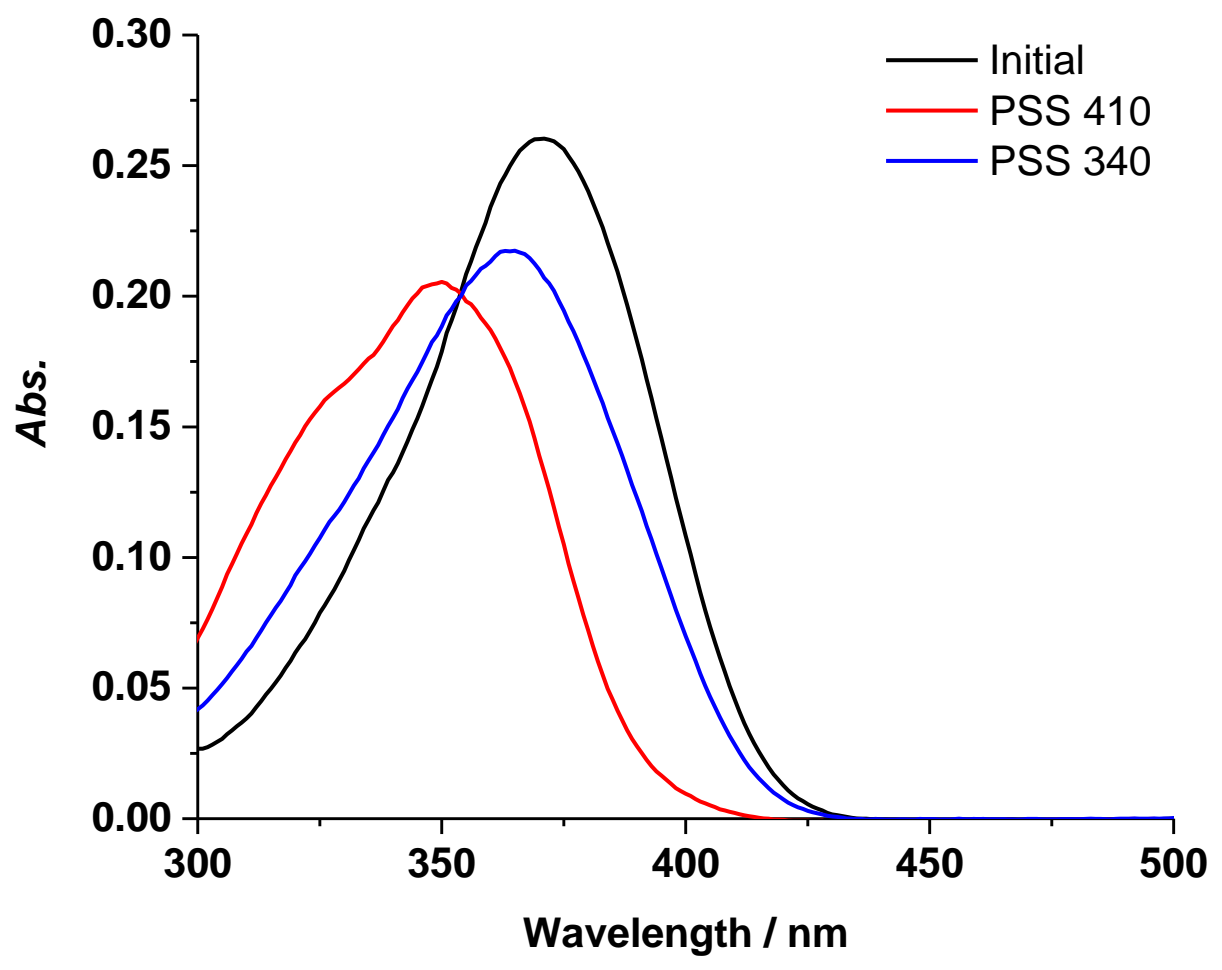

Figure S42. Light induced $Z / E$ isomerization of P1 $(4 \mu \mathrm{g} / \mathrm{mL})$ in $\mathrm{DCM}$ 


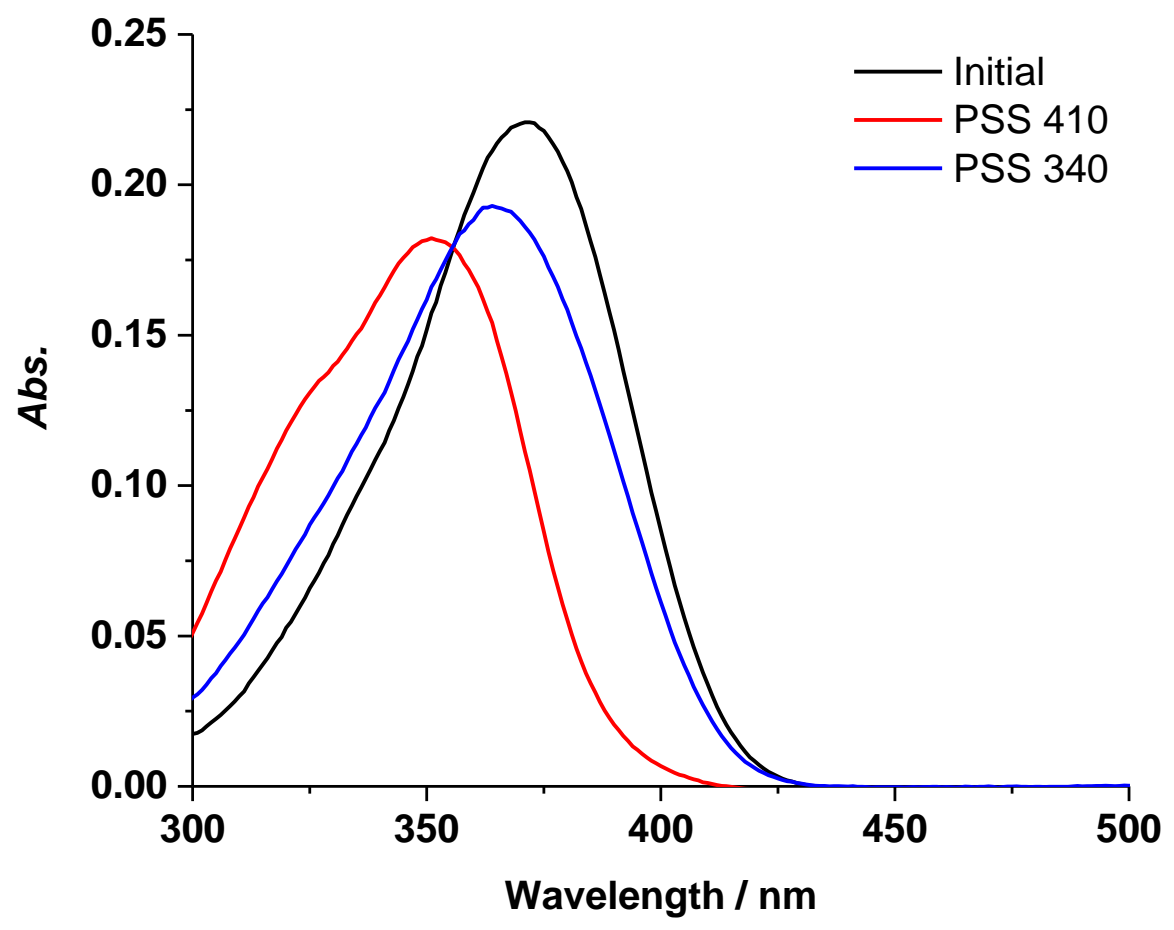

Figure S43. Light induced $Z / E$ isomerization of $\mathbf{P 2}(4 \mu \mathrm{g} / \mathrm{mL})$ in DCM

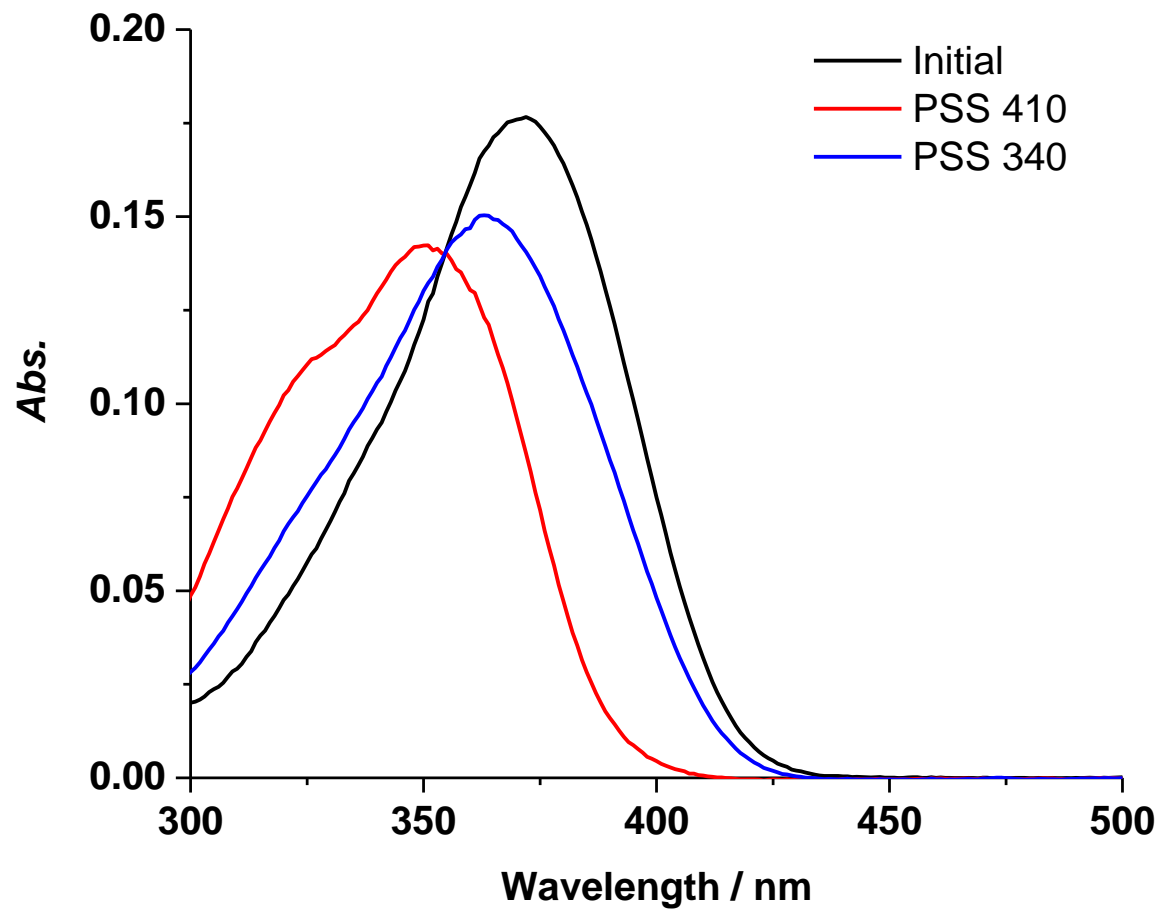

Figure S44. Light induced $Z / E$ isomerization of $\mathbf{P 3}(4 \mu \mathrm{g} / \mathrm{mL})$ in $\mathrm{DCM}$ 


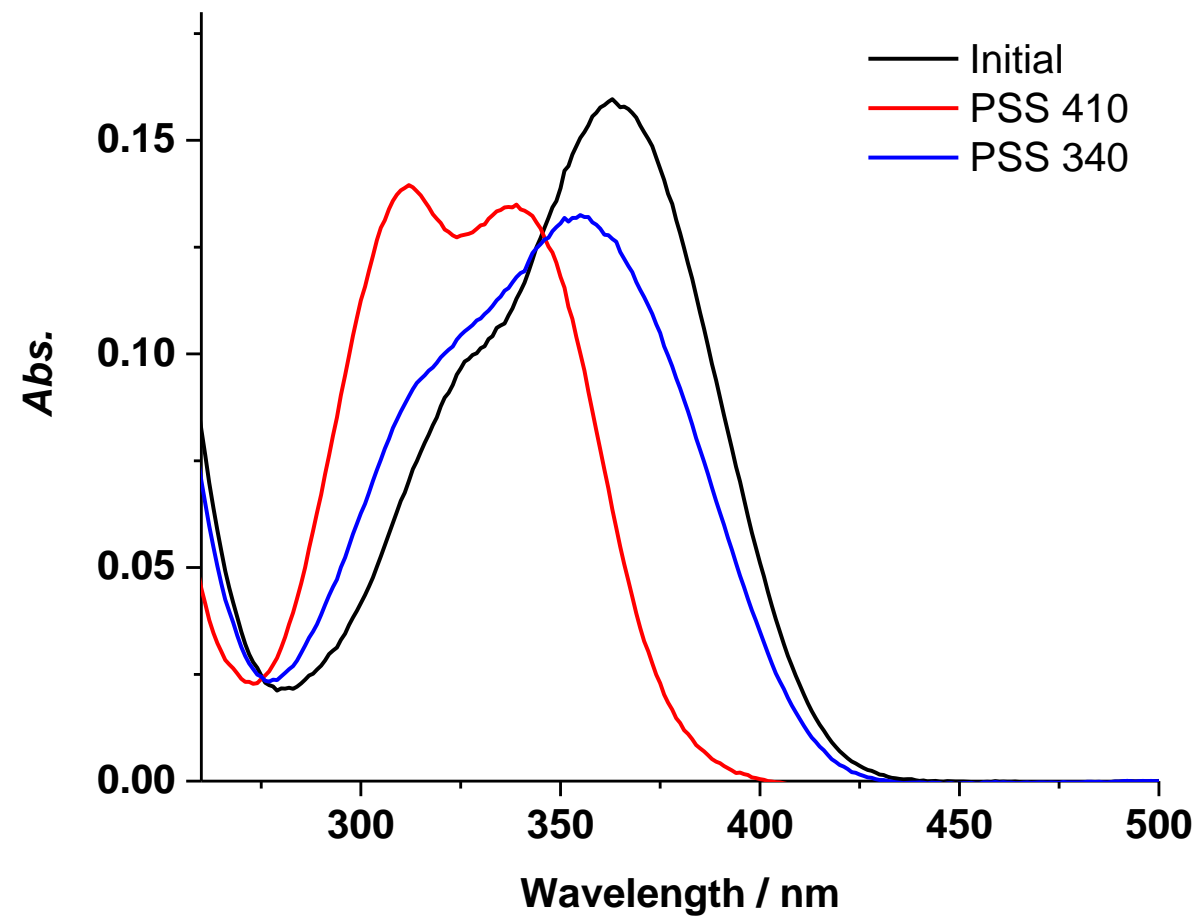

Figure S45. Light induced $Z / E$ isomerization of $\mathbf{P 4}(4 \mu \mathrm{g} / \mathrm{mL})$ in DCM

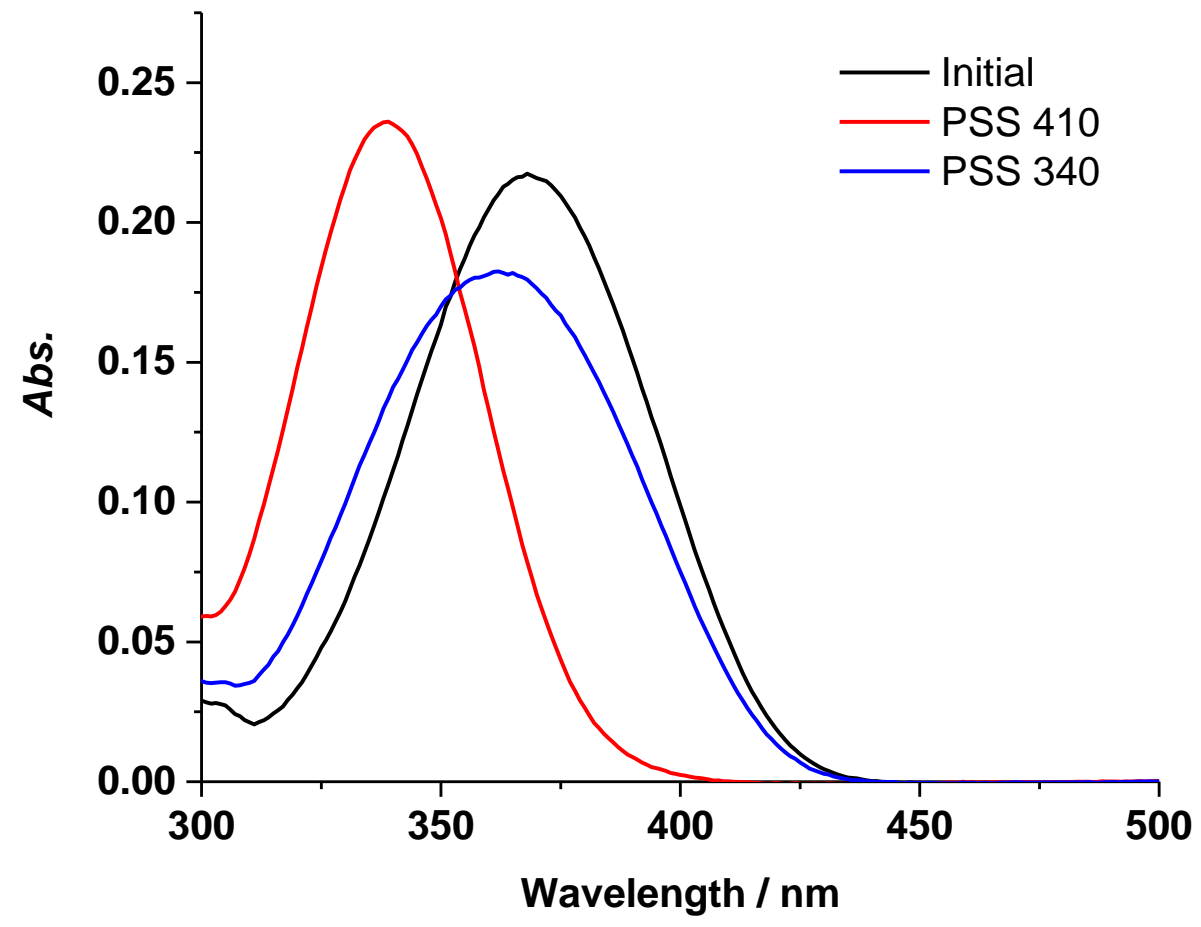

Figure S46. Light induced $Z / E$ isomerization of P5 $(4 \mu \mathrm{g} / \mathrm{mL})$ in DCM 


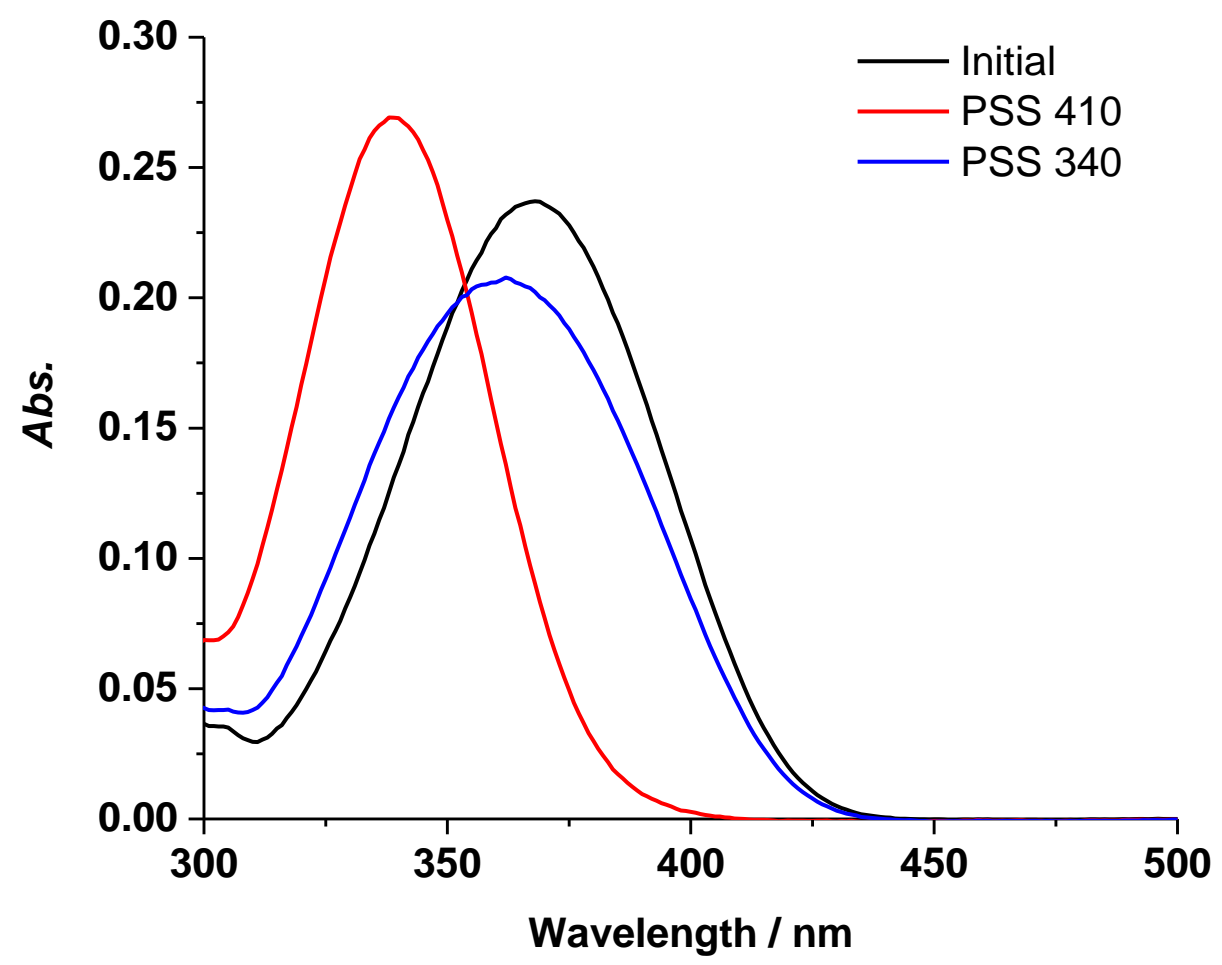

Figure S47. Light induced $Z / E$ isomerization of $\mathbf{P 6}(5 \mu \mathrm{g} / \mathrm{mL})$ in DCM

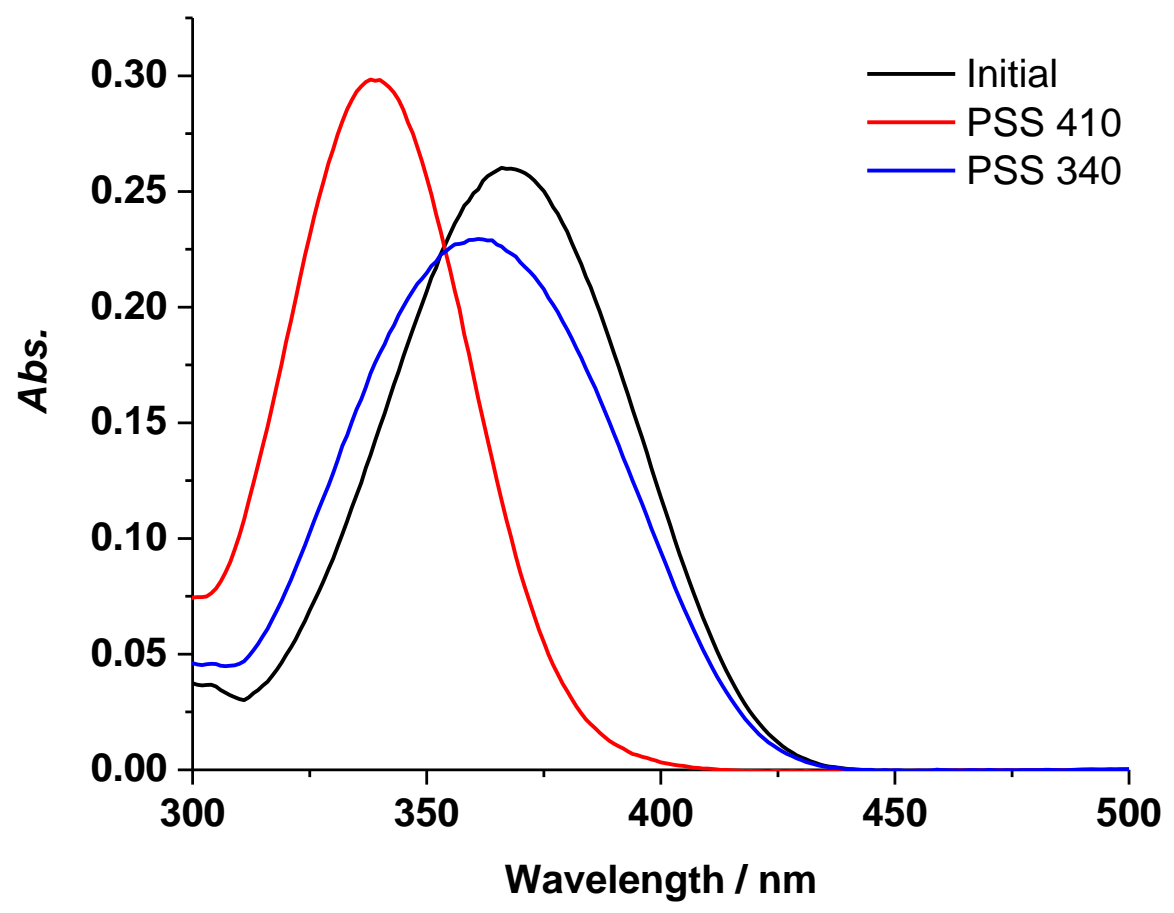

Figure S48. Light induced $Z / E$ isomerization of $\mathbf{P 7}(5 \mu \mathrm{g} / \mathrm{mL})$ in $\mathrm{DCM}$ 


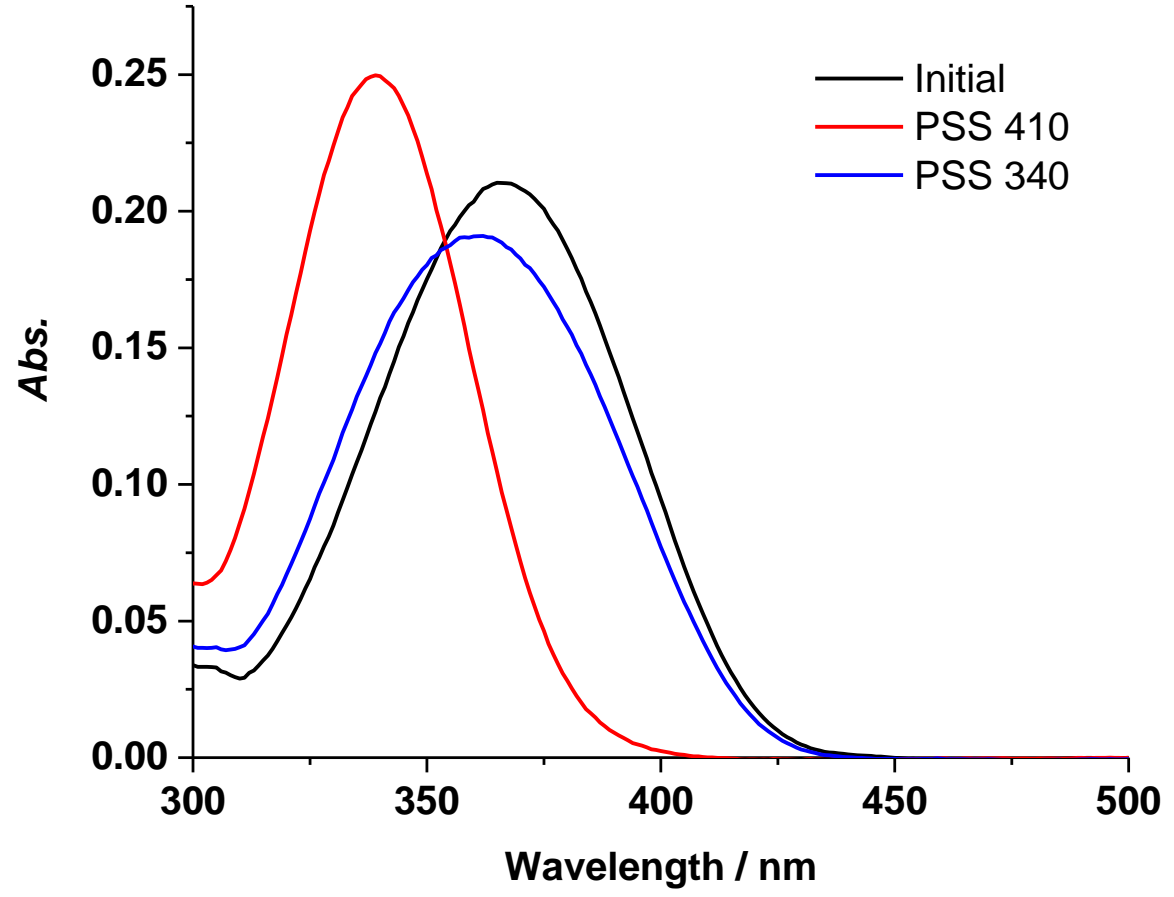

Figure S49. Light induced $Z / E$ isomerization of P8 $(5 \mu \mathrm{g} / \mathrm{mL})$ in DCM 


\section{Photoswitching of Thin Film Polymers}

Polymer solutions in DCM were prepared (ca. $1 \mathrm{mg} / 1 \mathrm{~mL}$ ) and then drop-casted onto quartz plates to form transparent thin films used in UV/Vis absorption measurements. The films were then irradiated, and corresponding UV spectra recorded.

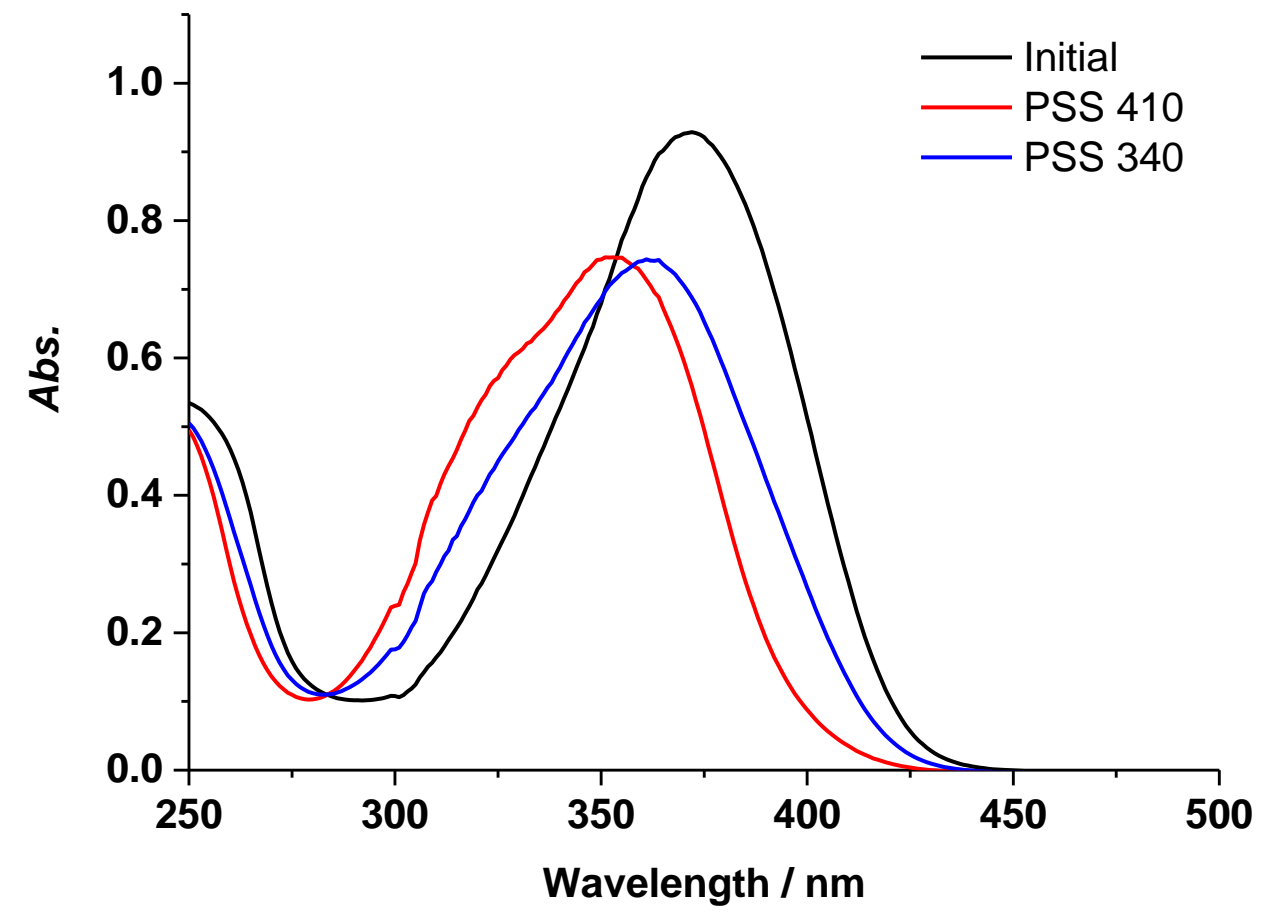

Figure S50. Light induced $Z / E$ isomerization of $\mathbf{P 1}$ in thin film state 


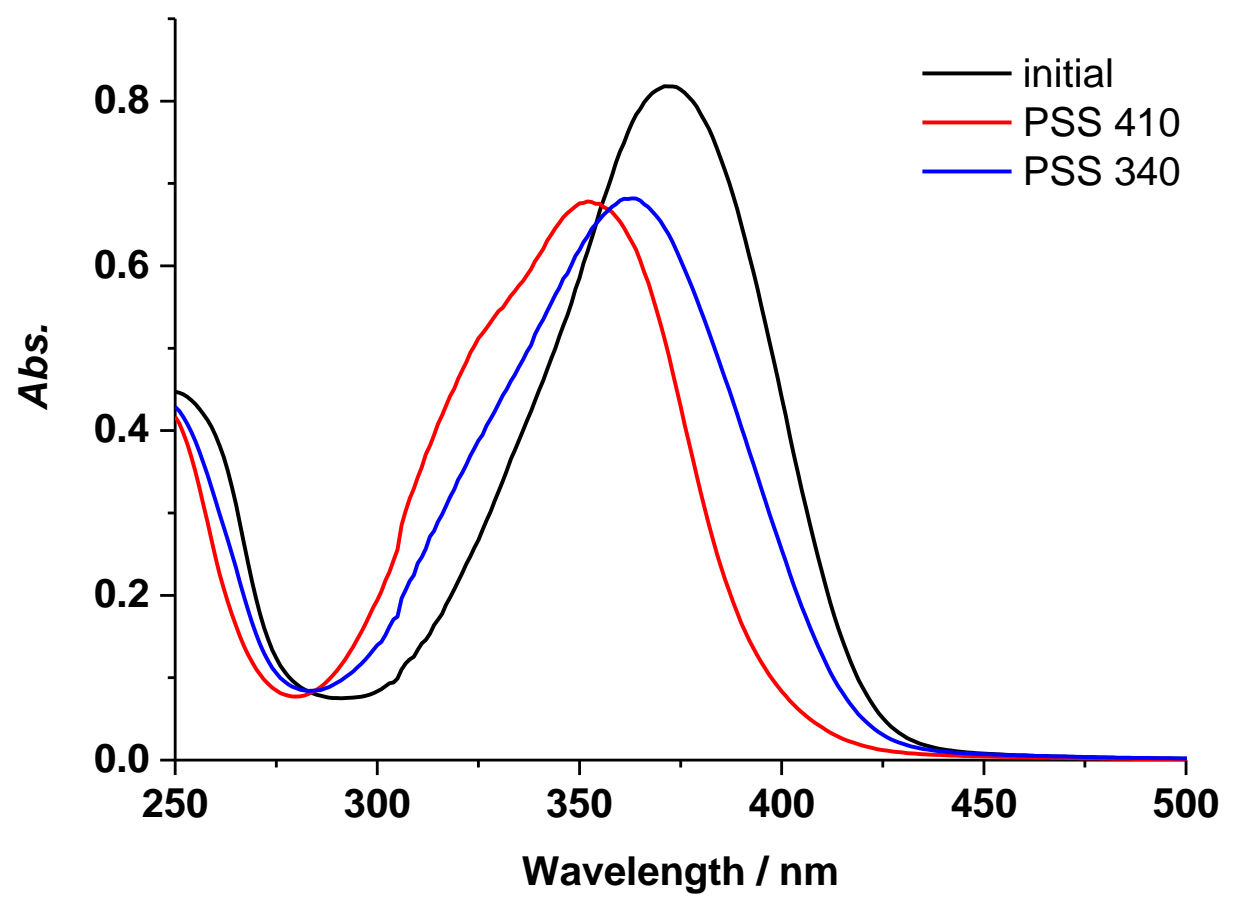

Figure S51. Light induced $Z / E$ isomerization of $\mathbf{P 2}$ in thin film state

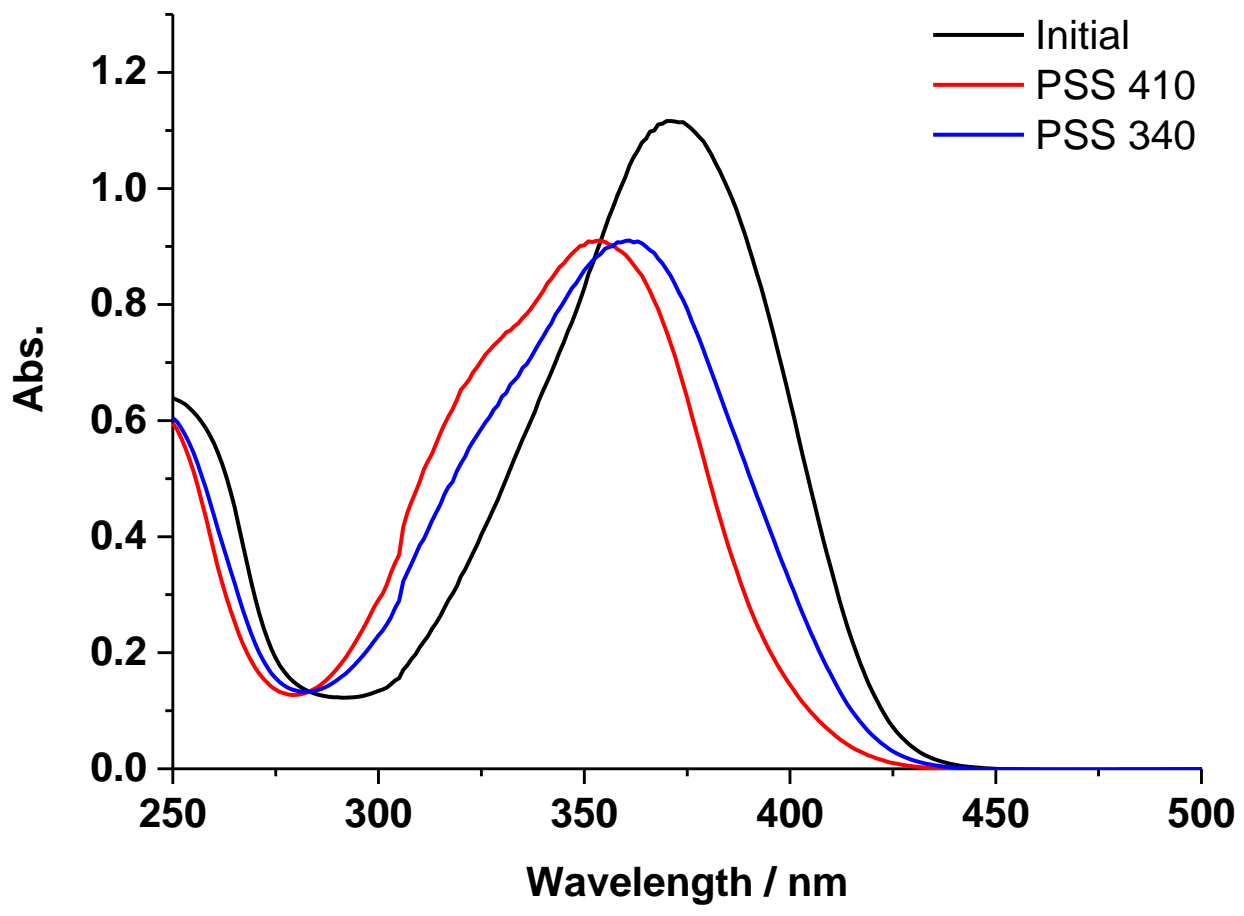

Figure S52. Light induced $Z / E$ isomerization of $\mathbf{P 3}$ in thin film state 


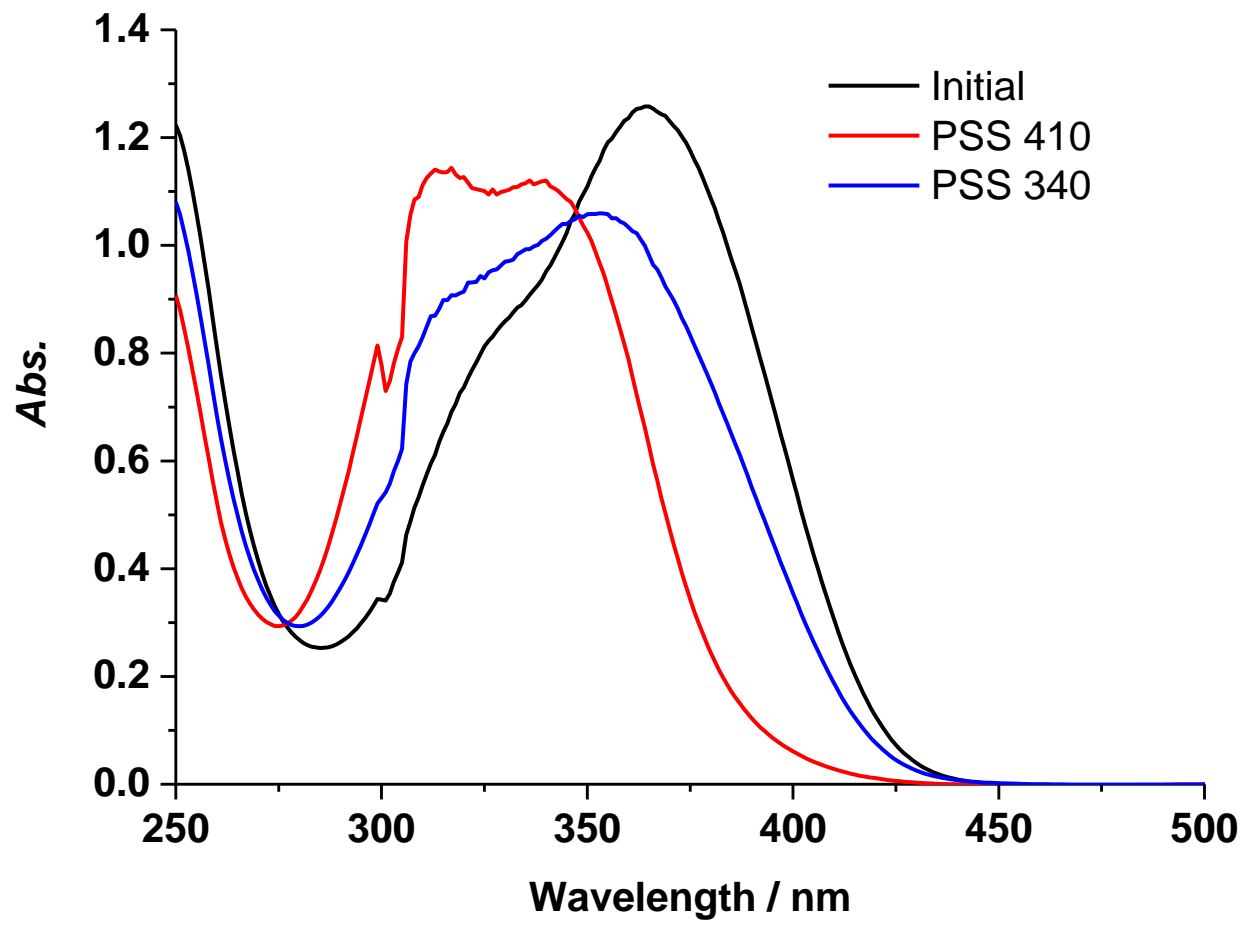

Figure S53. Light induced $Z / E$ isomerization of $\mathbf{P 4}$ in thin film state

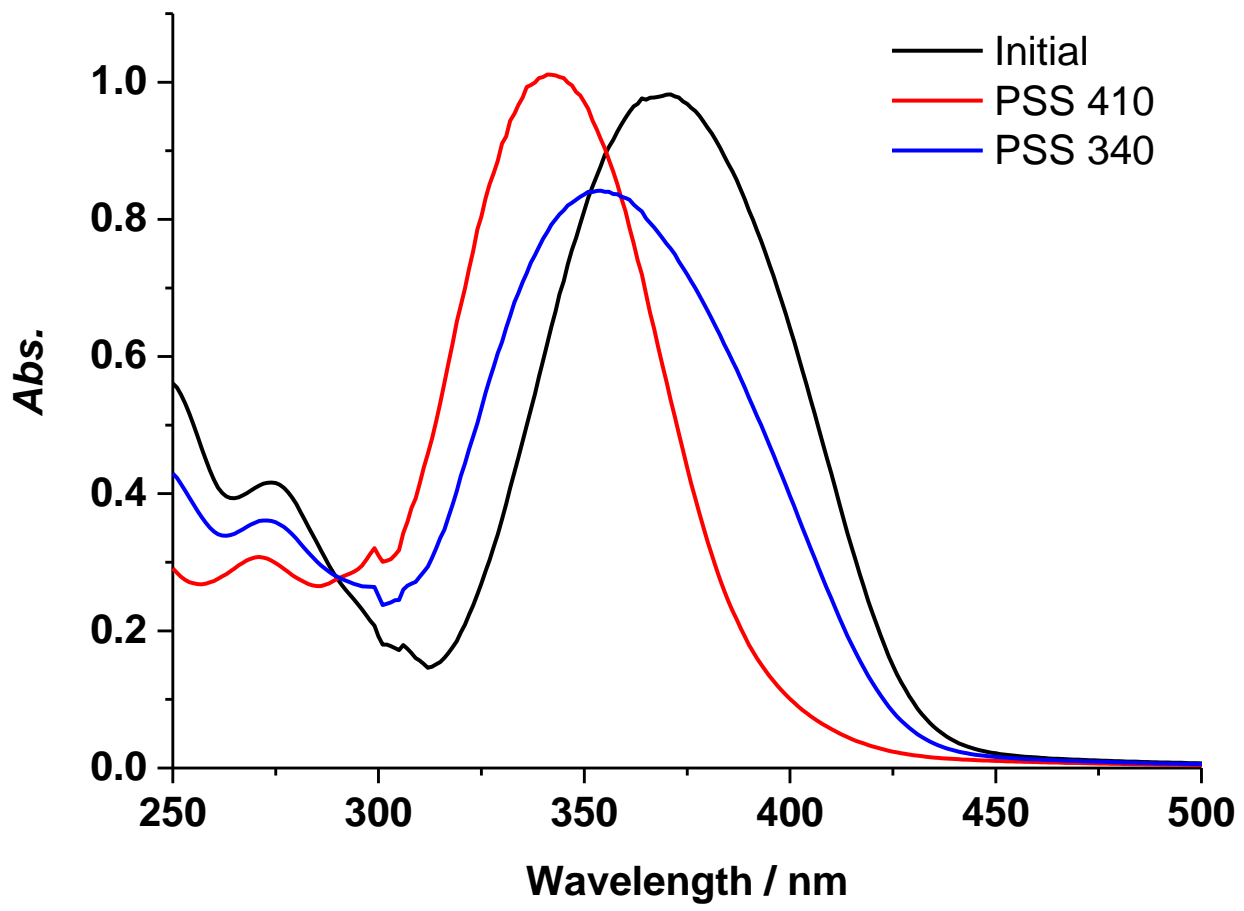

Figure S54. Light induced $Z / E$ isomerization of P5 in thin film state 


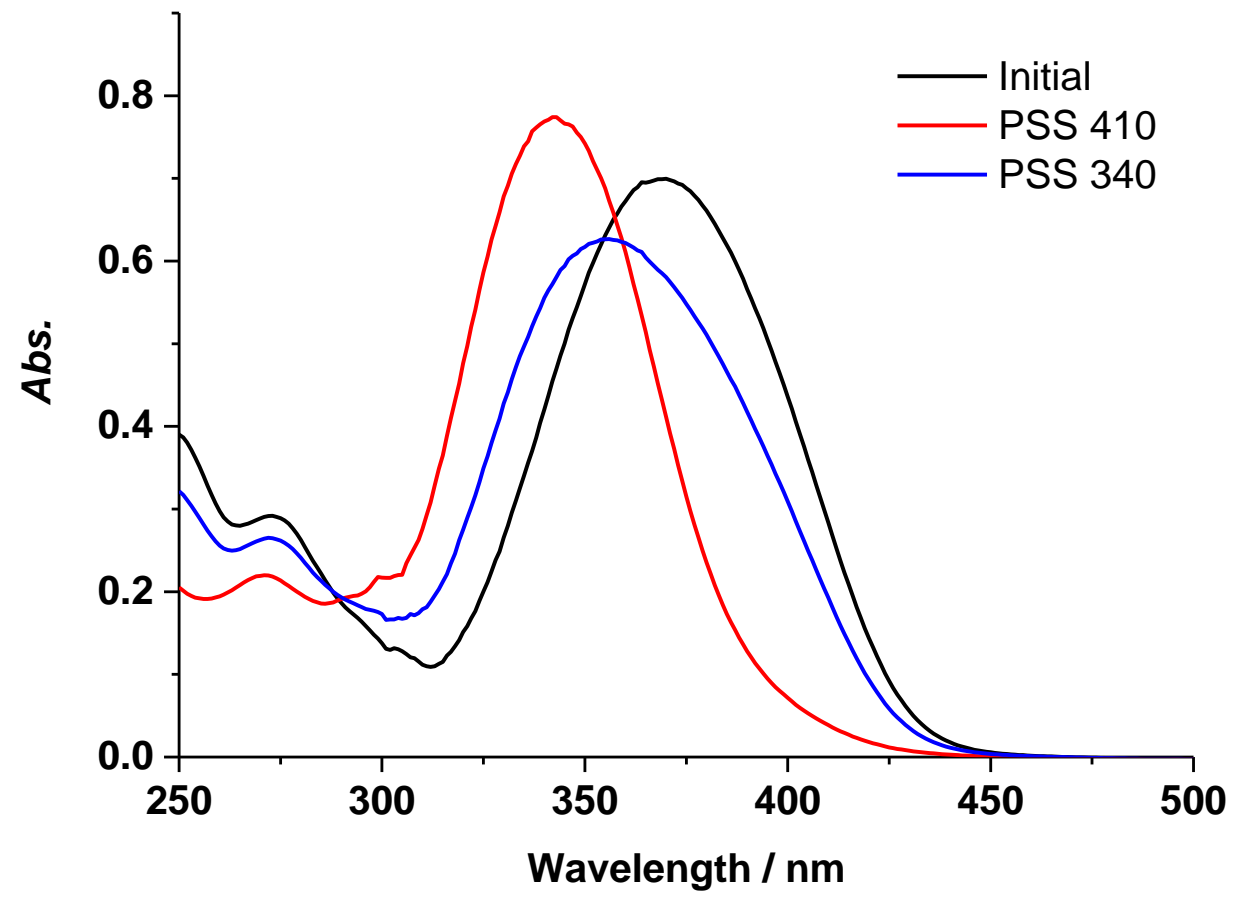

Figure S55. Light induced $Z / E$ isomerization of $\mathbf{P 6}$ in thin film state

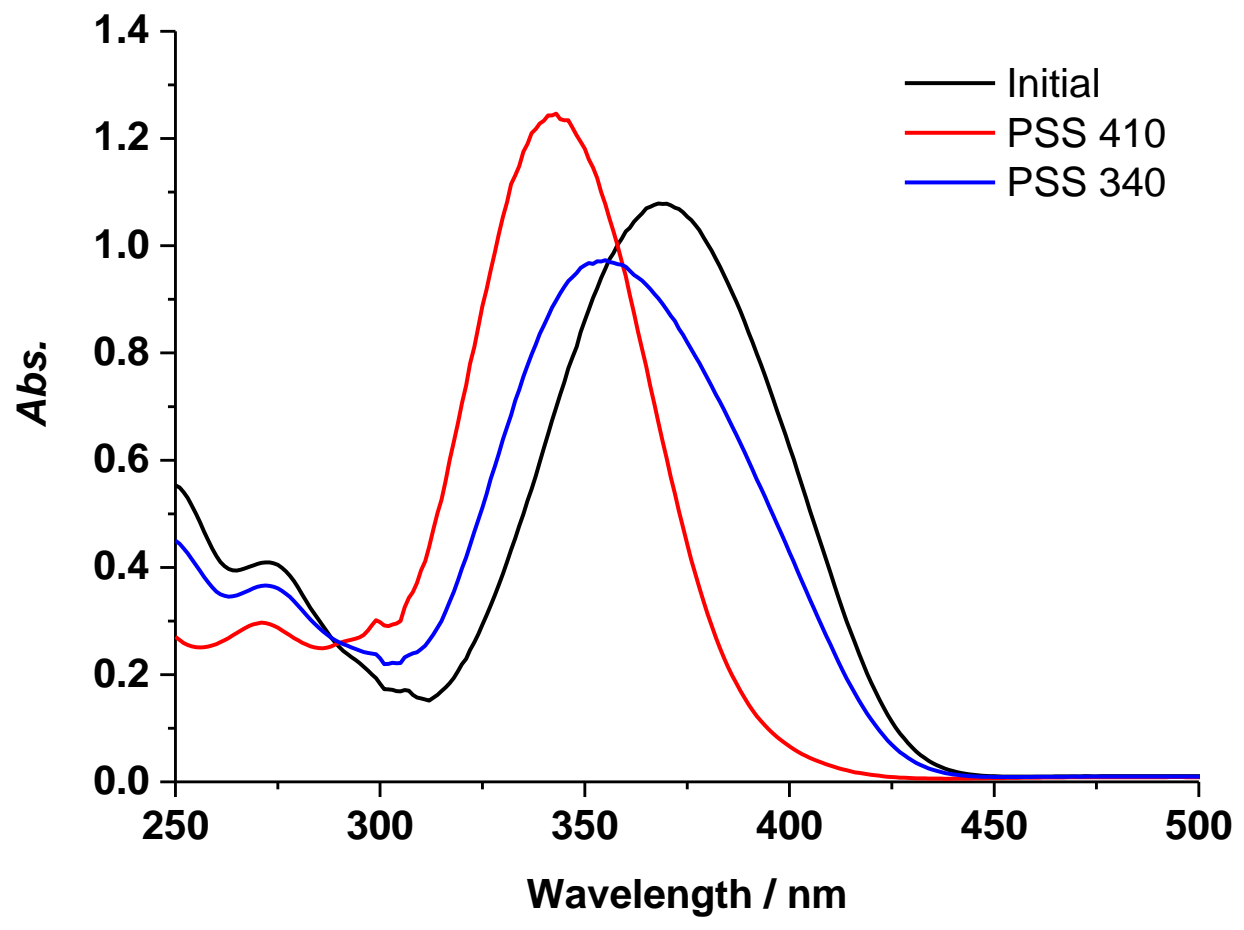

Figure S56. Light induced $Z / E$ isomerization of $\mathbf{P 7}$ in thin film state 


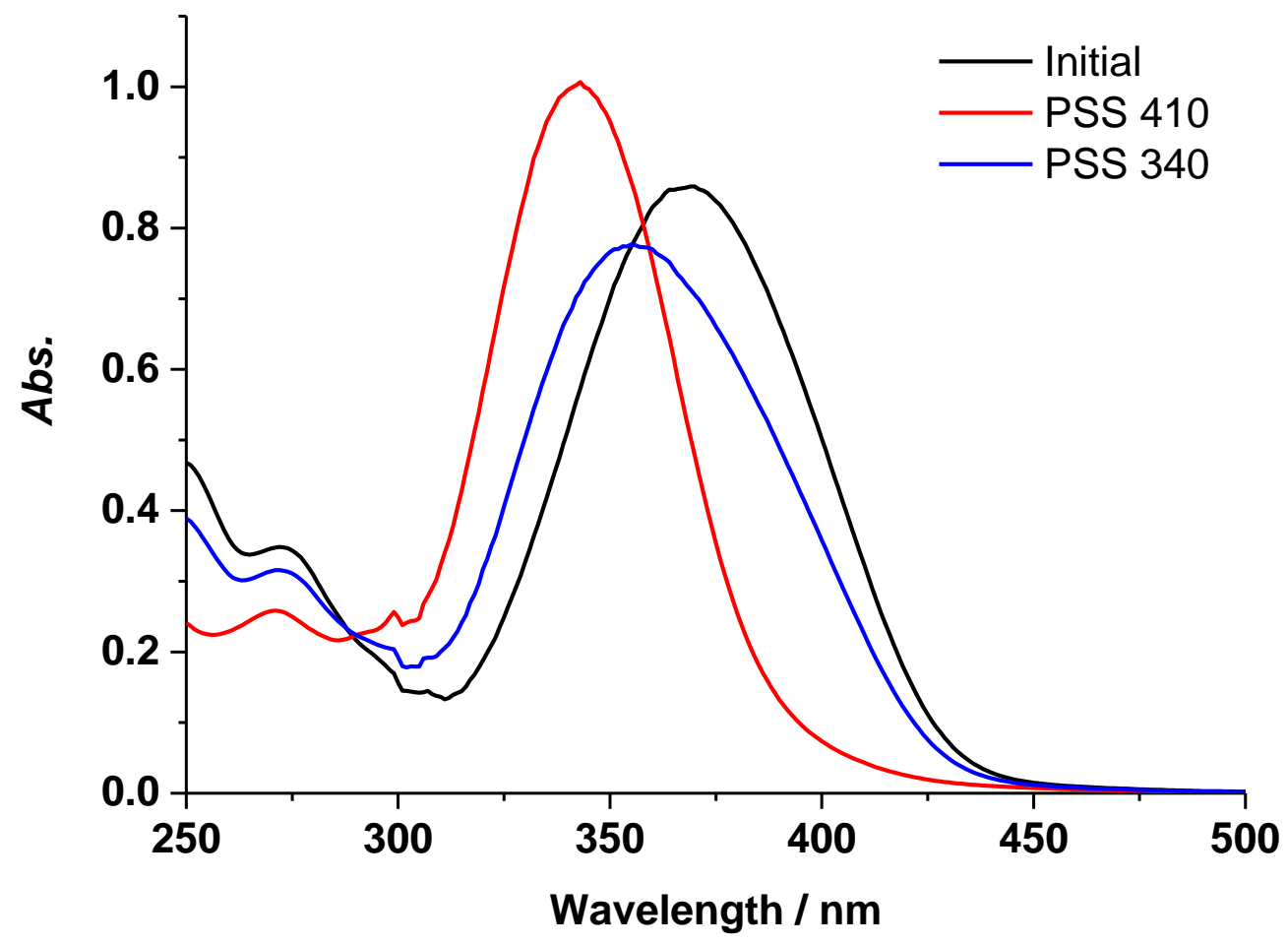

Figure S57. Light induced $Z / E$ isomerization of $\mathbf{P 8}$ in thin film state 


\section{Polymer Photoisomerization}

The polymer PSSs were determined upon continuous irradiation of the sample until no further isomerization was observed using ${ }^{1} \mathrm{H}$ NMR spectroscopy.

a) Initial State: $>99 \%$ Z
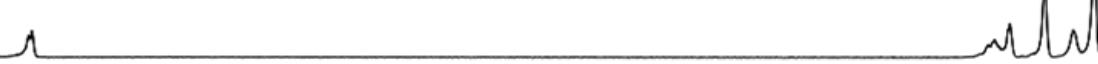

b) PSS 410: $94 \% E$

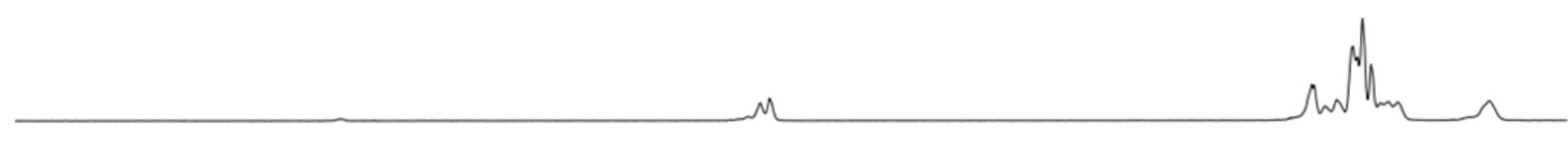

c) PSS 340: $77 \% Z$

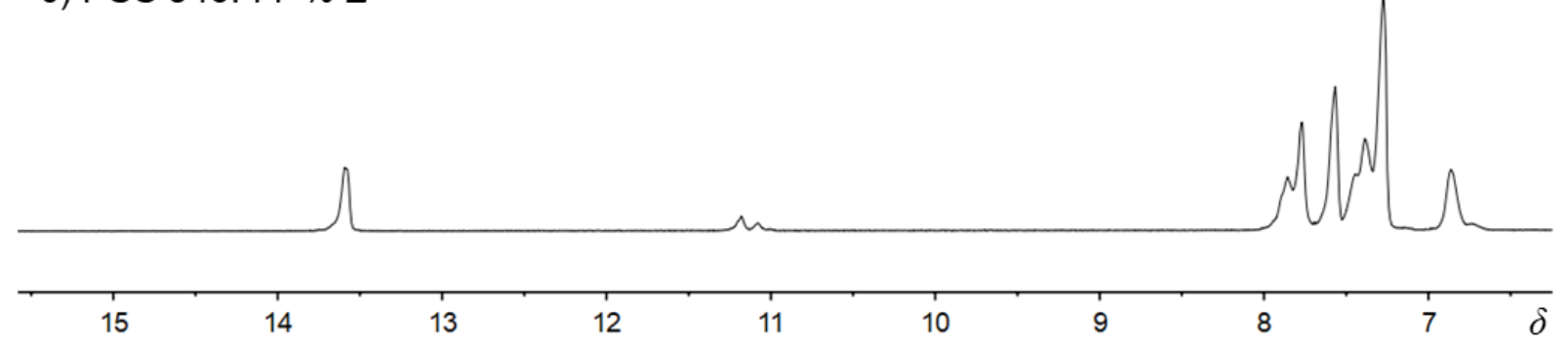

Figure S58. ${ }^{1} \mathrm{H}$ NMR spectra $\left(\mathrm{CD}_{2} \mathrm{Cl}_{2}, 298 \mathrm{~K}\right)$ of P1 a) before, and after b) $410 \mathrm{~nm}$ followed by c) $340 \mathrm{~nm}$ photo irradiation to reach the appropriate PSS. 
a) Initial State: $>99 \%$ Z

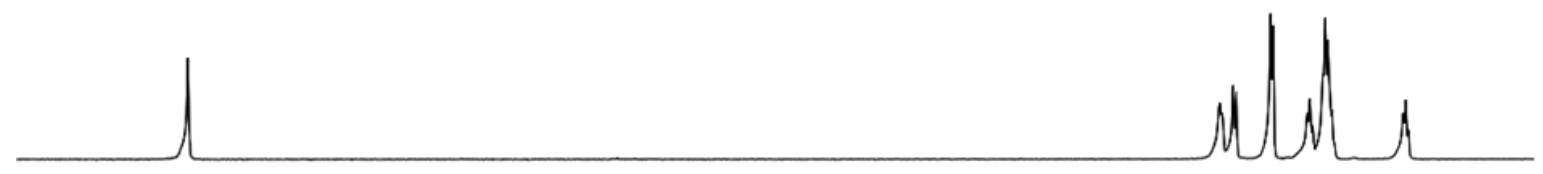

b) PSS 410: $95 \% E$

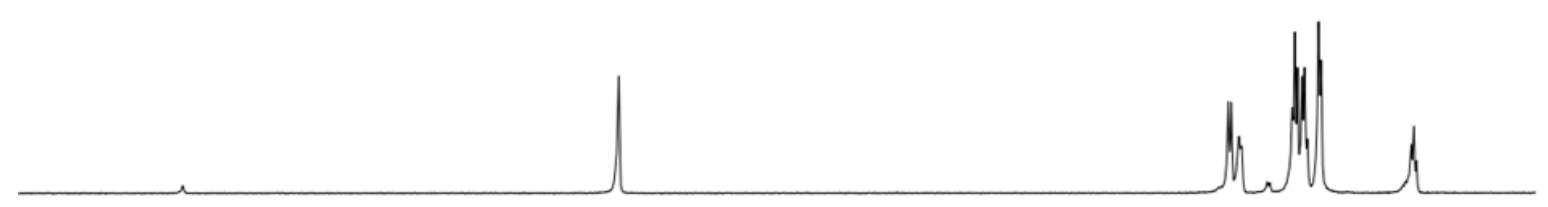

c) PSS $340: 77 \% Z$

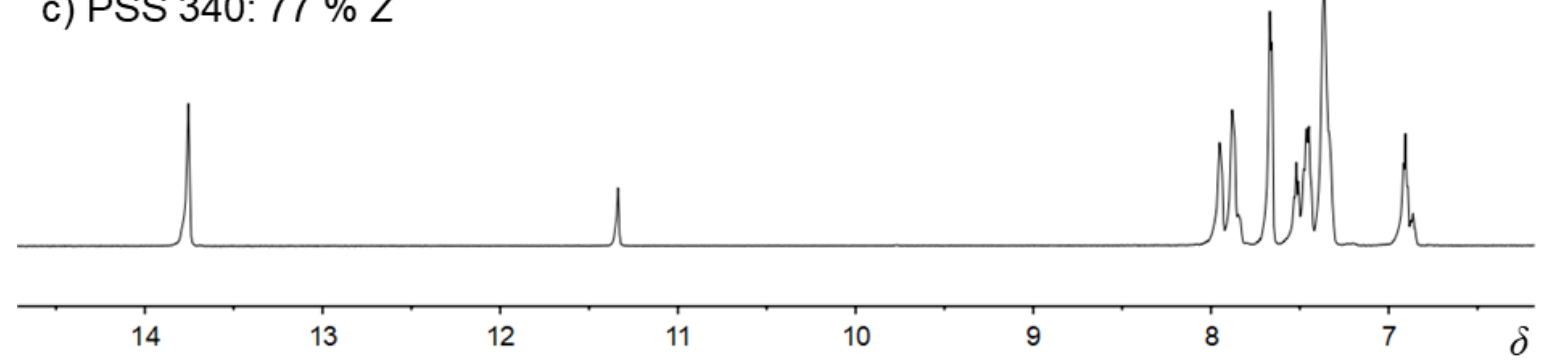

Figure S59. ${ }^{1} \mathrm{H}$ NMR spectra $\left(\mathrm{CD}_{2} \mathrm{Cl}_{2}, 298 \mathrm{~K}\right)$ of $\mathbf{P 2}$ a) before, and after b) $410 \mathrm{~nm}$ followed by c) $340 \mathrm{~nm}$ photo irradiation to reach the appropriate PSS.

a) Initial State: $>99 \% Z$

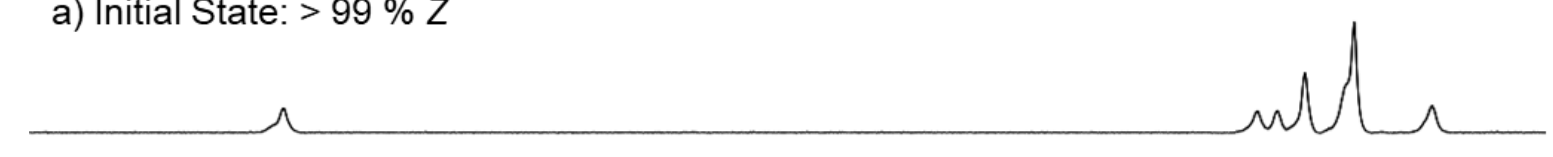

b) PSS 410: $95 \% E$

c) PSS 340: $72 \%$ Z

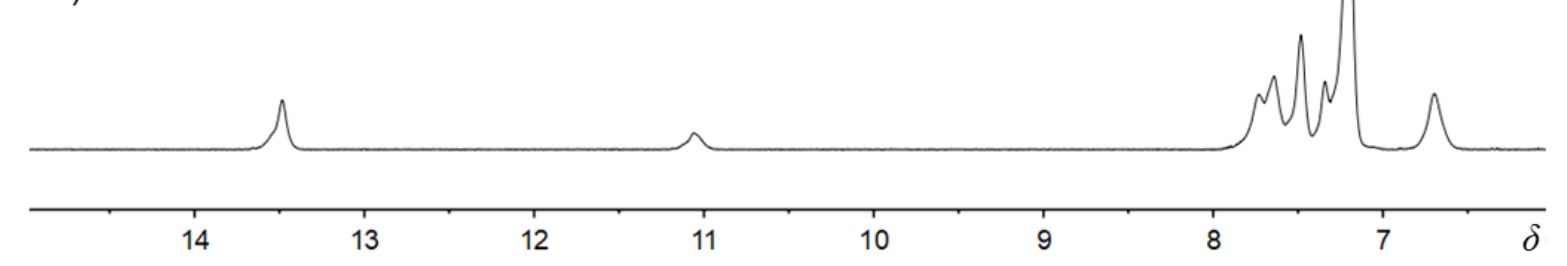

Figure S60. ${ }^{1} \mathrm{H}$ NMR spectra $\left(\mathrm{CD}_{2} \mathrm{Cl}_{2}, 298 \mathrm{~K}\right)$ of $\mathbf{P 3}$ a) before, and after b) $410 \mathrm{~nm}$ followed by c) $340 \mathrm{~nm}$ photo irradiation to reach the appropriate PSS. 
a) Initial State: $>99 \% Z$

b) PSS 410: $98 \% E$
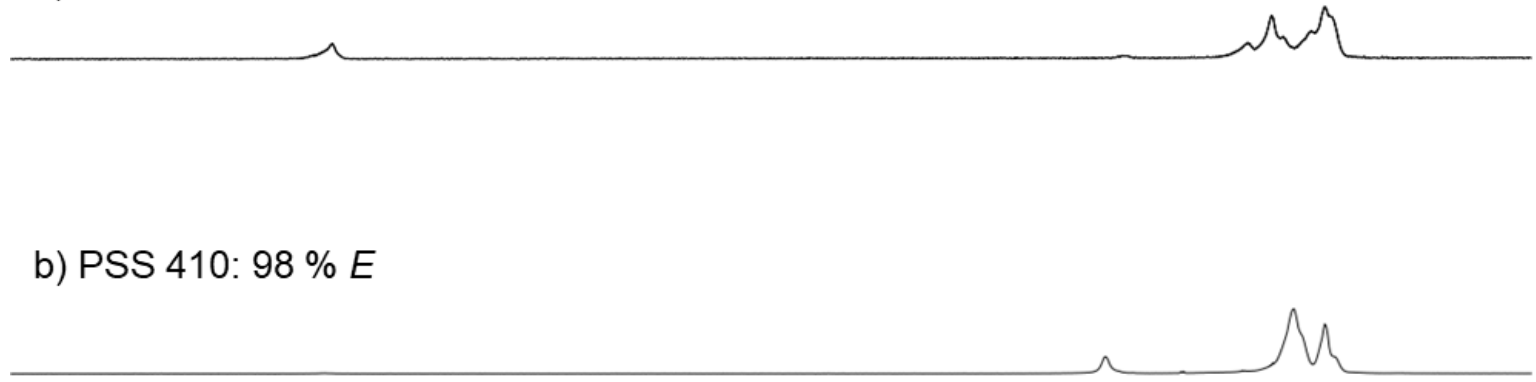

c) PSS $340: 68 \% Z$

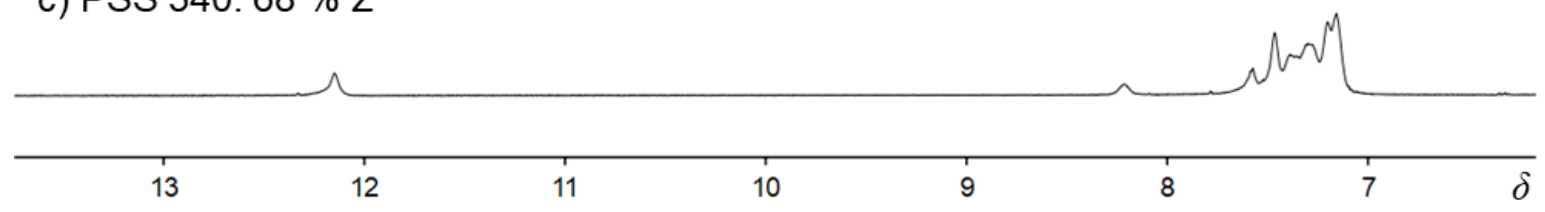

Figure S61. ${ }^{1} \mathrm{H}$ NMR spectra $\left(\mathrm{CD}_{2} \mathrm{Cl}_{2}, 298 \mathrm{~K}\right)$ of $\mathbf{P 4}$ a) before, and after b) $410 \mathrm{~nm}$ followed by c) $340 \mathrm{~nm}$ photo irradiation to reach the appropriate PSS.

a) Initial State: $>99 \%$ Z

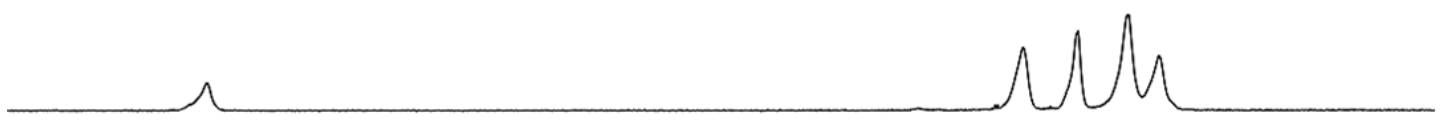

b) PSS 410: $98 \% E$

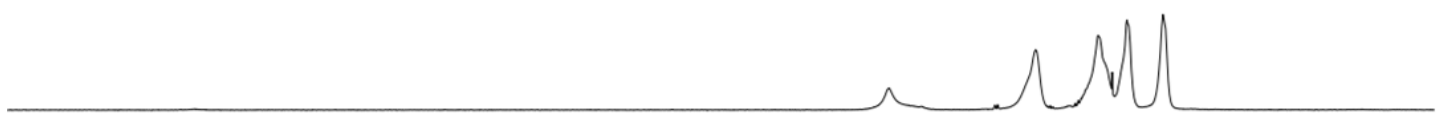

c) PSS $340: 80 \% Z$

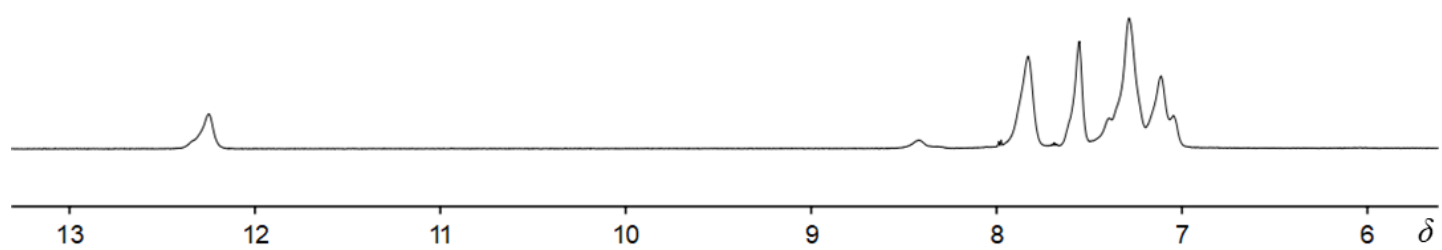

Figure S62. ${ }^{1} \mathrm{H}$ NMR spectra $\left(\mathrm{CD}_{2} \mathrm{Cl}_{2}, 298 \mathrm{~K}\right)$ of $\mathbf{P 6}$ a) before, and after b) $410 \mathrm{~nm}$ followed by c) $340 \mathrm{~nm}$ photo irradiation to reach the appropriate PSS 
a) Initial State: $>99 \%$ Z

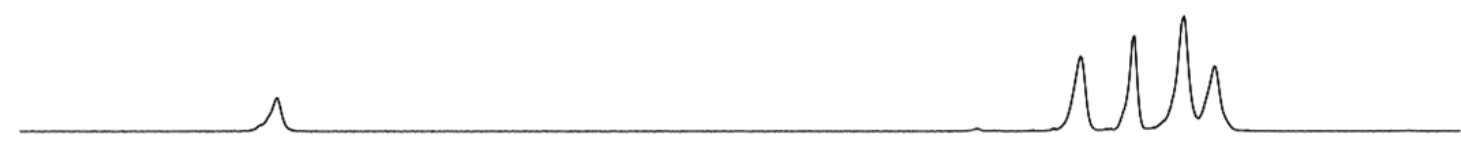

b) PSS 410: $98 \% E$

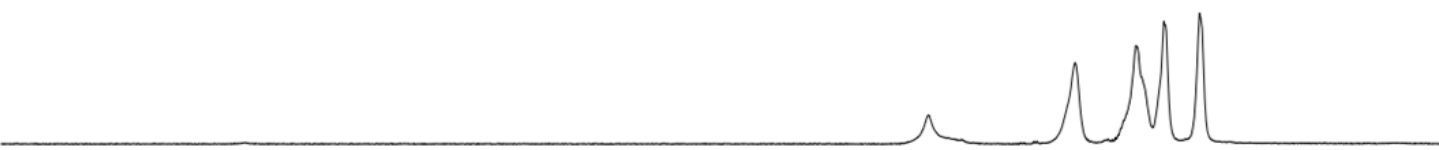

c) PSS 340: $80 \%$ Z

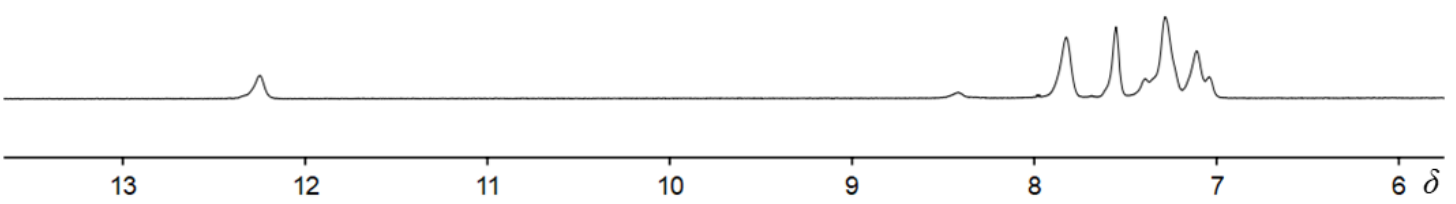

Figure S63. ${ }^{1} \mathrm{H}$ NMR spectra $\left(\mathrm{CD}_{2} \mathrm{Cl}_{2}, 298 \mathrm{~K}\right)$ of $\mathbf{P 7}$ a) before, and after b) $410 \mathrm{~nm}$ followed by c) $340 \mathrm{~nm}$ photo irradiation to reach the appropriate PSS

a) Initial State: > $99 \%$ Z

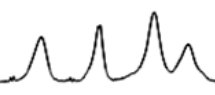

b) PSS 410: $98 \% E$

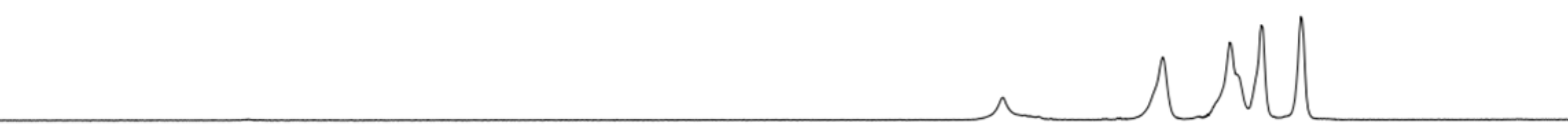

c) PSS 340: $80 \%$ Z

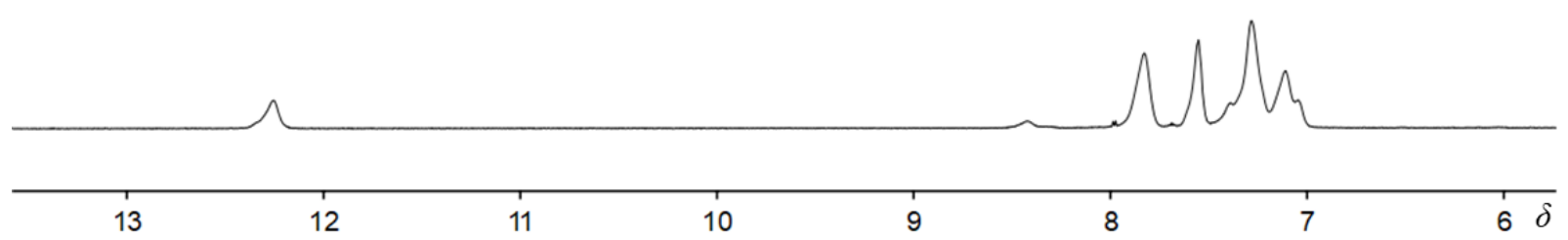

Figure S64. ${ }^{1} \mathrm{H}$ NMR spectra $\left(\mathrm{CD}_{2} \mathrm{Cl}_{2}, 298 \mathrm{~K}\right)$ of P8 a) before, and after b) $410 \mathrm{~nm}$ followed by c) $340 \mathrm{~nm}$ photo irradiation to reach the appropriate PSS 


\section{Photoisomerization Quantum Yield of Polymers}

The molar photon flux $I_{0}$ at 340 and $410 \mathrm{~nm}$ were determined using chemical actinometry. ${ }^{\mathrm{S} 3}$ A $0.5 \mathrm{~mL}\left(=V_{0}\right)$ solution of potassium ferrioxalate in $0.05 \mathrm{M} \mathrm{H}_{2} \mathrm{SO}_{4}$ was placed into a $1.0 \mathrm{~cm}$ cuvette and irradiated for 30 seconds $\left(=t_{0}\right)$. The irradiated solution was combined with 3.5 equiv. of ferrozine and stirred under dark for an hour. The resulting solution, containing reddish-purple $[\mathrm{Fe}(\text { ferrozine }) 3]^{2+}$ complex was diluted by a factor of $n$, and its absorbance was measured at 563 $\mathrm{nm}\left(A_{563}\right)$, where its molar absorption coefficient ( $\left.\varepsilon_{563}\right)$ is $27,900 \mathrm{~cm}^{-1} \mathrm{M}^{-1}$. The molar photon flux of the light source at different wavelengths was determined using Eq. 1.

$$
I_{0}\left(\text { quanta } \cdot s^{-1}\right)=\frac{A_{563} \cdot n \cdot N_{A} \cdot V_{0}}{\varepsilon_{563} \cdot l \cdot t_{0} \cdot \phi_{\lambda}} \quad \text { Eq. } 1
$$

where $l$ indicates the length of cuvette; $\phi_{\lambda}$ stands for the quantum yield of the photo-reduction of $\mathrm{Fe}(\mathrm{III})$ oxalate induced by the light source $\left(\phi_{340}=1.25, \phi_{410}=1.14\right)$; and $N_{\mathrm{A}}$ stands for Avogadro's number.

The photoisomerization quantum yields of hydrazone $\mathbf{1}$ were measured according to a previously reported method. ${ }^{\mathrm{S} 3}$

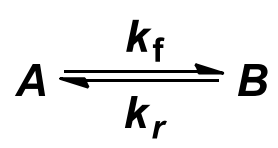

In a photochemical reaction, species $\mathbf{A}$ absorbs light to generate the product $\mathbf{B}$.

The rate law for the formation of species $\mathbf{A}$ is as follows:

$$
C_{A}=\frac{C_{\text {total }} \cdot k_{r}}{k_{f}+k_{r}}+\frac{C_{\text {total }} \cdot k_{f}}{k_{f}+k_{r}} e^{-\left(k_{f}+k_{r}\right) t} \quad \text { Eq. } 2
$$

$C_{A}$ is proportional to the absorbance of species $\mathbf{A}$. An exponential fit of absorbance as a function of time gives the observed rate constant $k_{o b s}=k_{f}+k_{r}$. For hydrazone switch $\mathbf{1}$, the thermal relaxation is slow at room temperature (Fig. S13) so $k_{A \rightarrow B} \approx k_{o b s}$. 


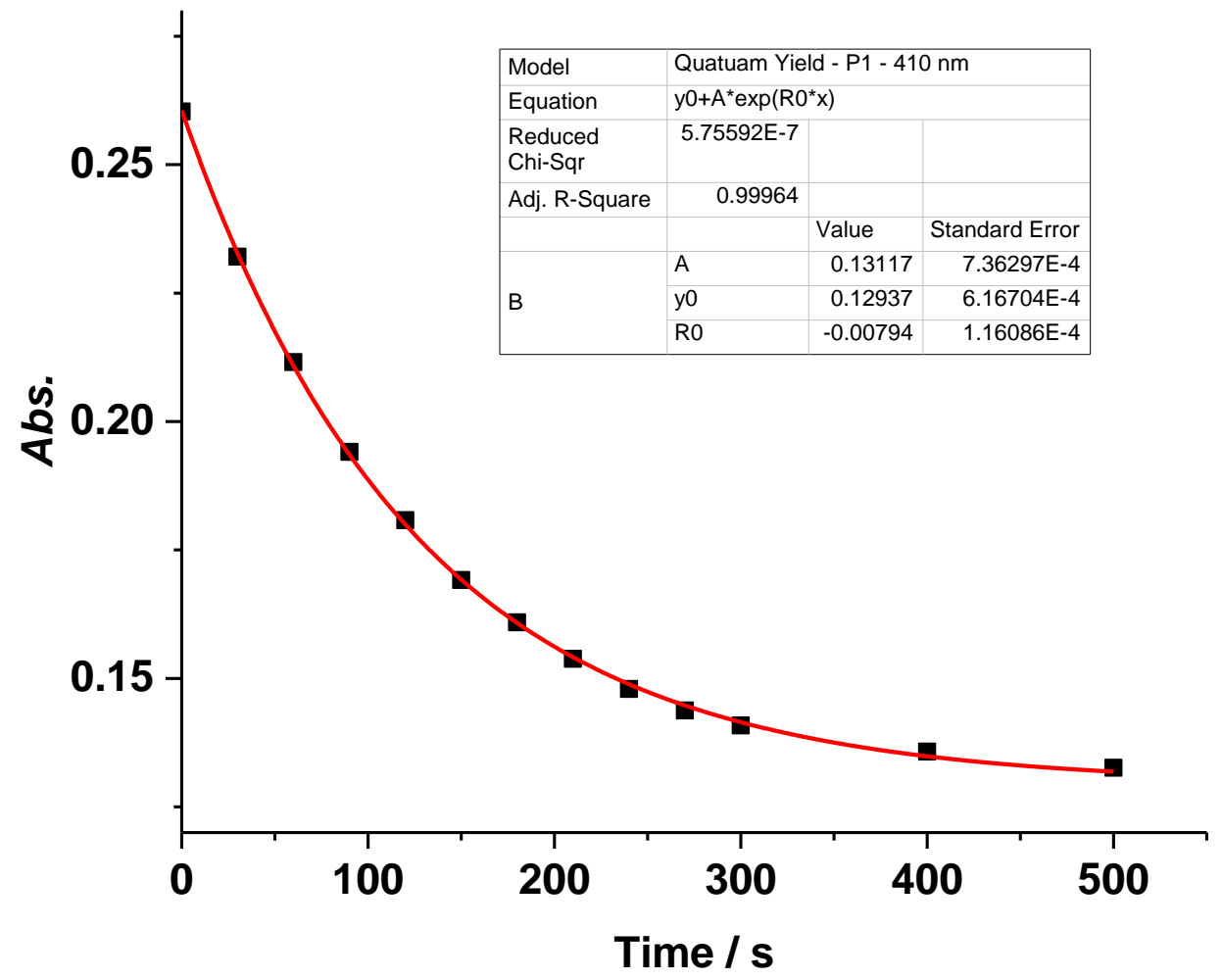

Figure S65. Kinetics for the photoisomerization (irradiation at $410 \mathrm{~nm}$ ) of P1- $Z$ to P1- $E$ in DCM $\left(1 \times 10^{-5} \mathrm{M}\right)$ at $298 \mathrm{~K}$; the plot is of absorption $(\lambda=371 \mathrm{~nm})$ as a function of time. $\varepsilon_{1}-Z @ 410 \mathrm{~nm}=$ $4600 \mathrm{M}^{-1} \cdot \mathrm{cm}^{-1}$ (for one repeating unit) was used for quantum yield calculations. The photoisomerization quantum yield was calculated to be $1.6 \pm 0.1 \%$ based on three consecutive measurements. 


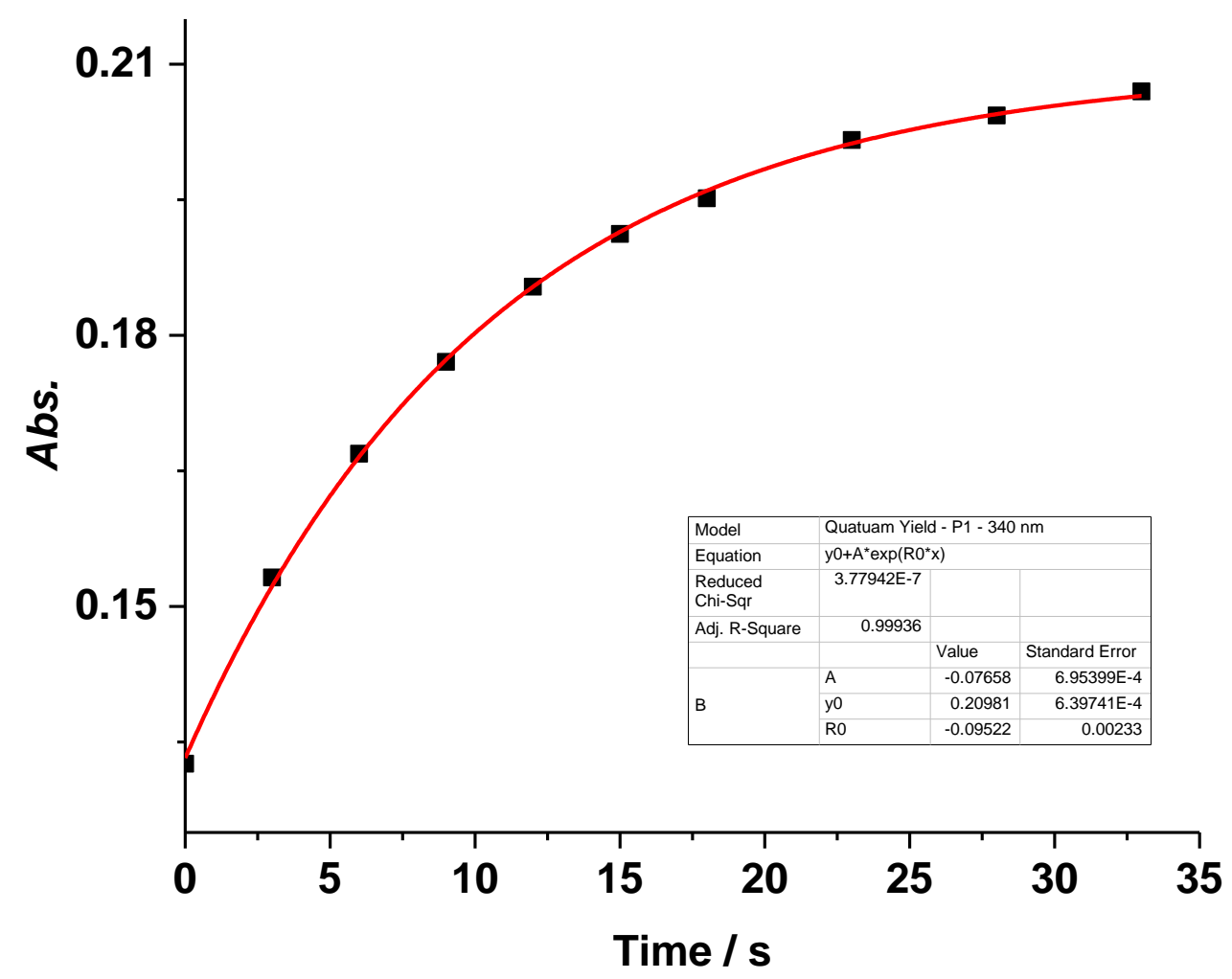

Figure S66. Kinetics for the photoisomerization (irradiation at $340 \mathrm{~nm}$ ) of P1- $E$ to P1- $Z$ in DCM $\left(1 \times 10^{-5} \mathrm{M}\right)$ at $298 \mathrm{~K}$; the plot is of absorption $(\lambda=371 \mathrm{~nm})$ as a function of time. $\varepsilon_{1-Z} @ 340 \mathrm{~nm}=$ $19200 \mathrm{M}^{-1} \cdot \mathrm{cm}^{-1}$ (for one repeating unit) was used for quantum yield calculations. The photoisomerization quantum yield was calculated to be $4.2 \pm 0.3 \%$ based on three consecutive measurements. 


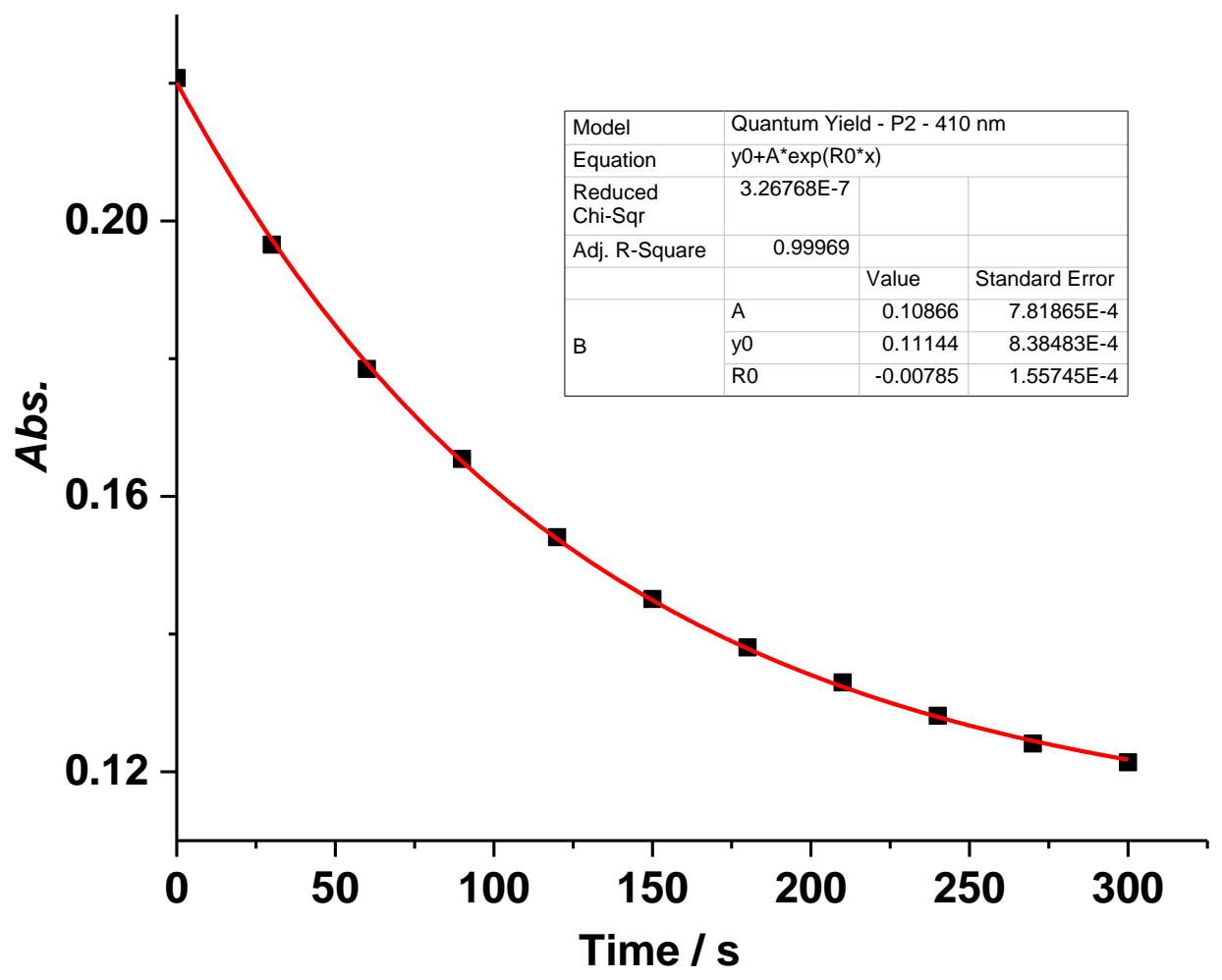

Figure S67. Kinetics for the photoisomerization (irradiation at $410 \mathrm{~nm}$ ) of $\mathbf{P 2}-Z$ to $\mathbf{P 2}-E$ in DCM $\left(1 \times 10^{-5} \mathrm{M}\right)$ at $298 \mathrm{~K}$; the plot is of absorption $(\lambda=371 \mathrm{~nm})$ as a function of time. $\varepsilon_{1-Z @ 410 \mathrm{~nm}}=$ $3500 \mathrm{M}^{-1} \cdot \mathrm{cm}^{-1}$ (for one repeating unit) was used for quantum yield calculations. The photoisomerization quantum yield was calculated to be $1.6 \pm 0.1 \%$ based on three consecutive measurements. 


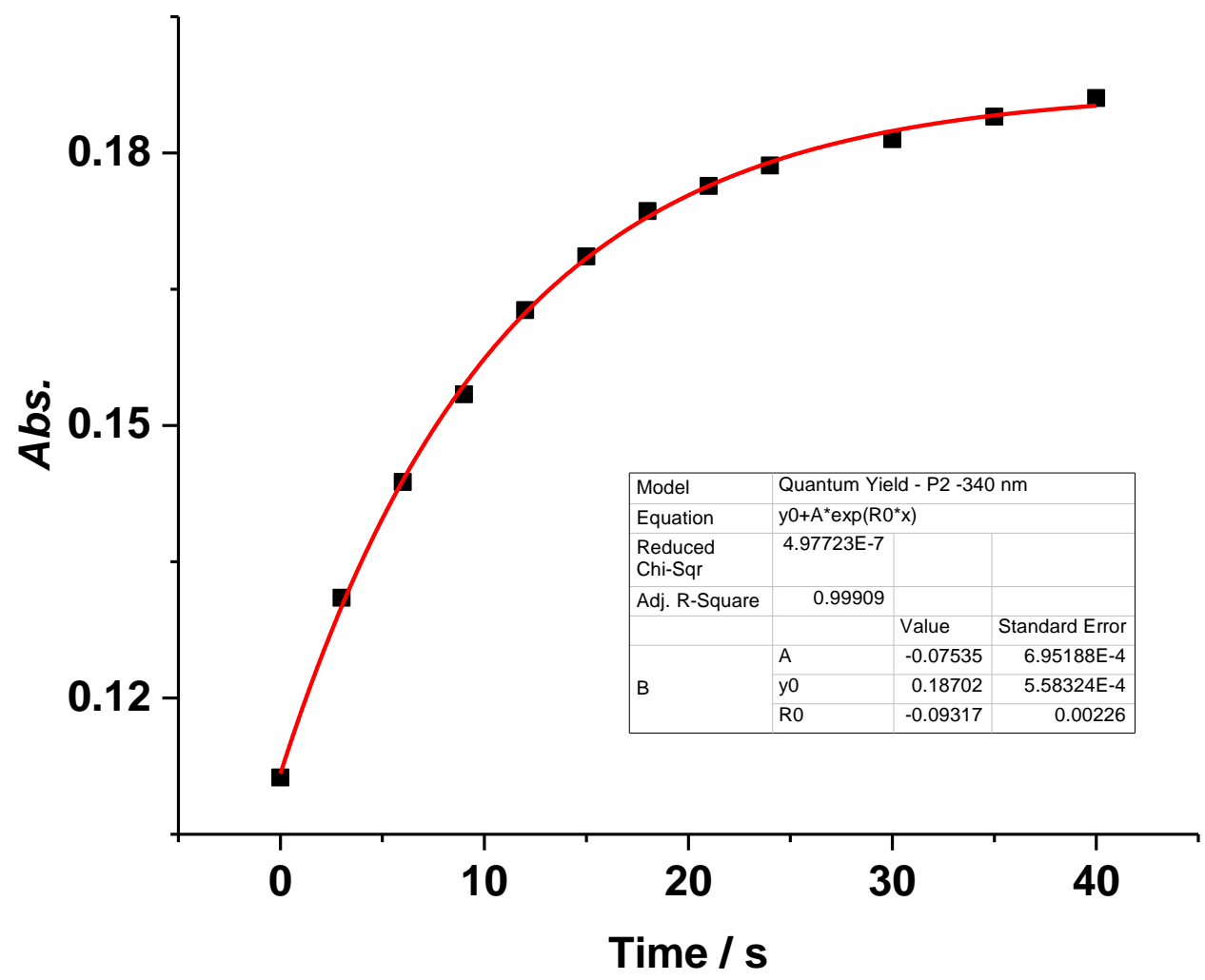

Figure S68. Kinetics for the photoisomerization (irradiation at $340 \mathrm{~nm}$ ) of $\mathbf{P 2}-E$ to $\mathbf{P 2}-Z$ in DCM $\left(1 \times 10^{-5} \mathrm{M}\right)$ at $298 \mathrm{~K}$; the plot is of absorption $(\lambda=371 \mathrm{~nm})$ as a function of time. $\varepsilon_{1}-Z @ 340 \mathrm{~nm}=$ $16600 \mathrm{M}^{-1} \cdot \mathrm{cm}^{-1}$ (for one repeating unit) was used for quantum yield calculations. The photoisomerization quantum yield was calculated to be $3.7 \pm 0.8 \%$ based on three consecutive measurements. 


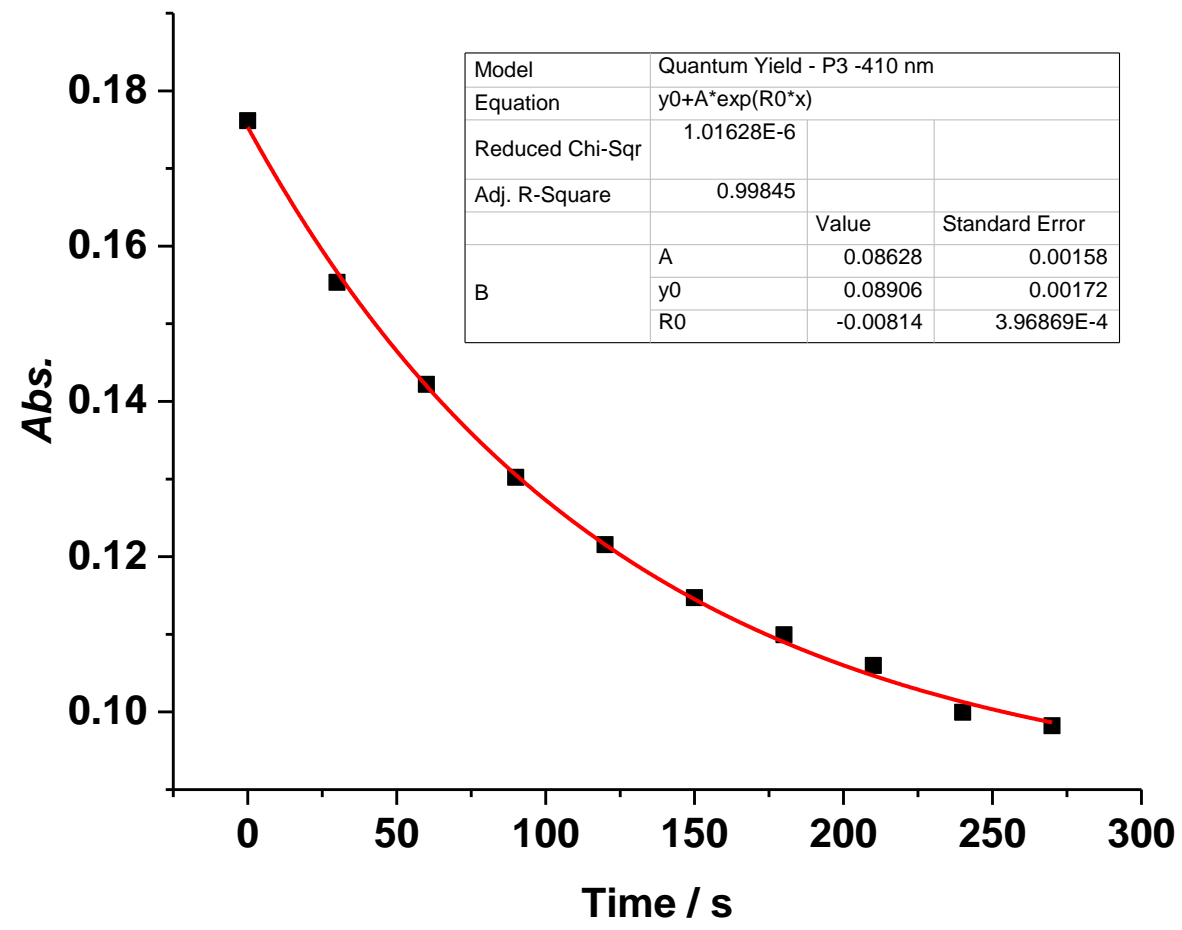

Figure S69. Kinetics for the photoisomerization (irradiation at $410 \mathrm{~nm}$ ) of P3- $Z$ to P3- $E$ in DCM $\left(1 \times 10^{-5} \mathrm{M}\right)$ at $298 \mathrm{~K}$; the plot is of absorption $(\lambda=371 \mathrm{~nm})$ as a function of time. $\varepsilon_{1}-Z @ 410 \mathrm{~nm}=$ $3200 \mathrm{M}^{-1} \cdot \mathrm{cm}^{-1}$ (for one repeating unit) was used for quantum yield calculations. The photoisomerization quantum yield was calculated to be $2.3 \pm 0.1 \%$ based on three consecutive measurements. 


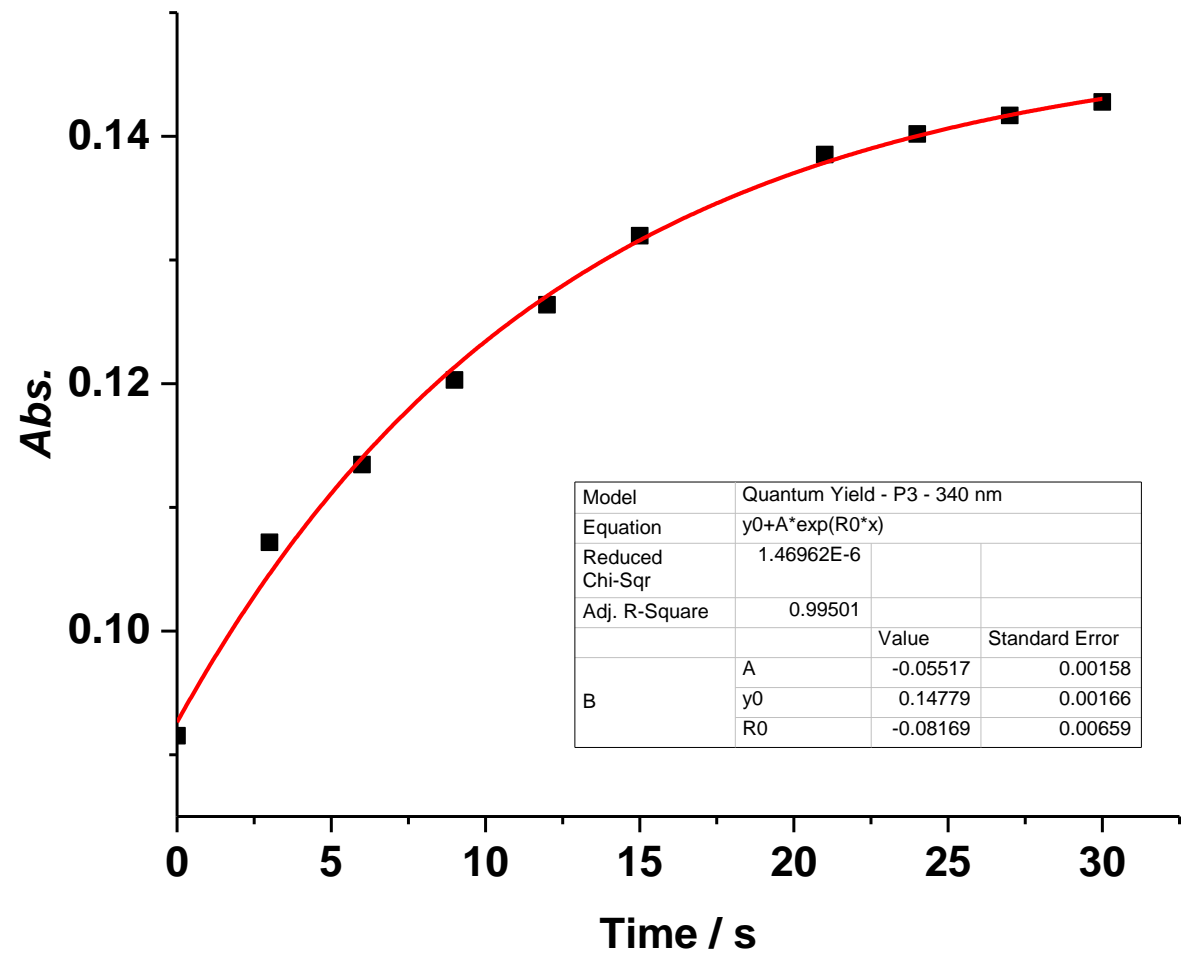

Figure S70. Kinetics for the photoisomerization (irradiation at $340 \mathrm{~nm}$ ) of P3- $E$ to P3- $Z$ in DCM $\left(1 \times 10^{-5} \mathrm{M}\right)$ at $298 \mathrm{~K}$; the plot is of absorption $(\lambda=371 \mathrm{~nm})$ as a function of time. $\varepsilon 1-Z @ 340 \mathrm{~nm}=$ $13100 \mathrm{M}^{-1} \cdot \mathrm{cm}^{-1}$ (for one repeating unit) was used for quantum yield calculations. The photoisomerization quantum yield was calculated to be $5.2 \pm 0.6 \%$ based on three consecutive measurements. 


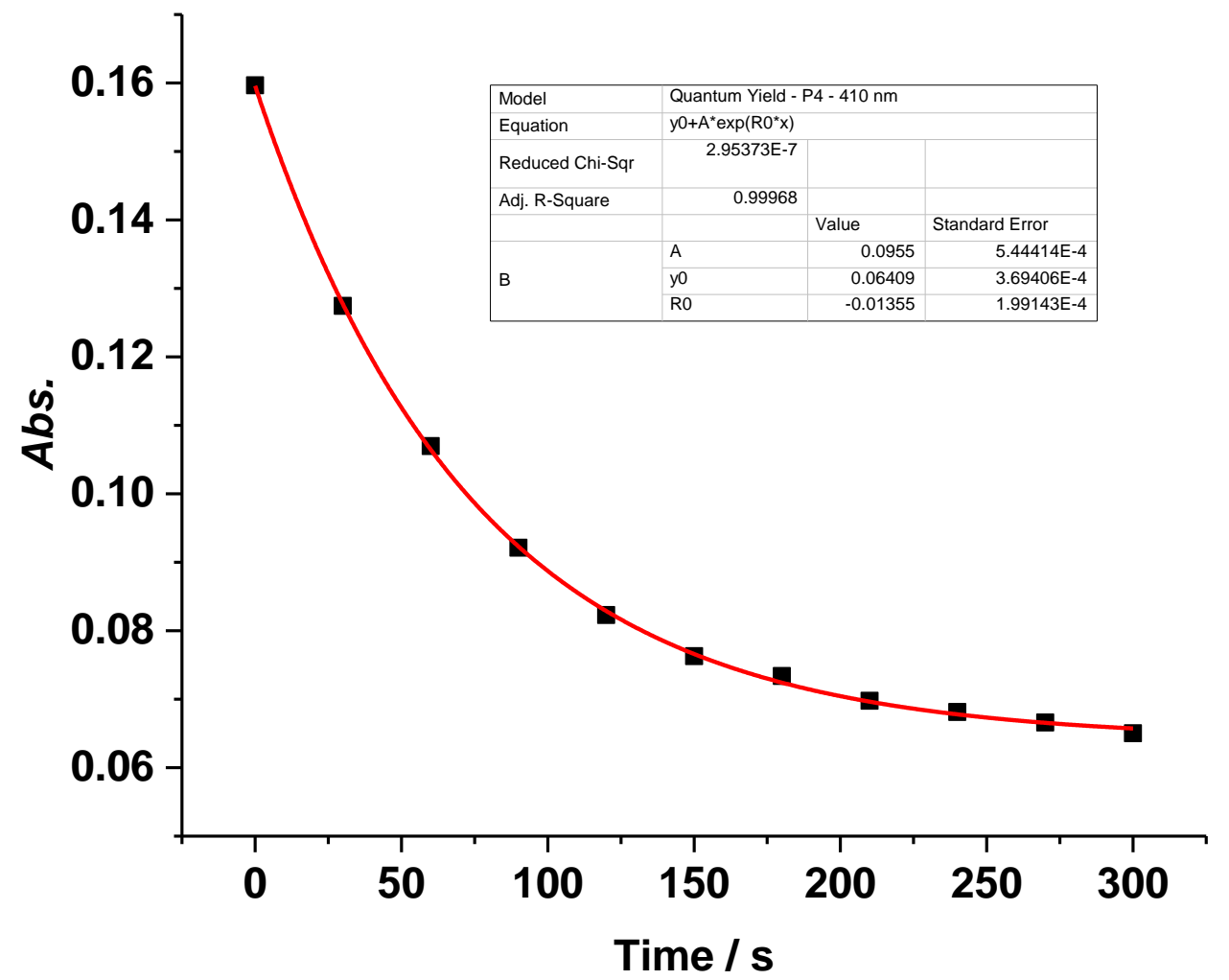

Figure S71. Kinetics for the photoisomerization (irradiation at $410 \mathrm{~nm}$ ) of P4- $Z$ to $\mathbf{P 4}-E$ in DCM $\left(1 \times 10^{-5} \mathrm{M}\right)$ at $298 \mathrm{~K}$; the plot is of absorption $(\lambda=371 \mathrm{~nm})$ as a function of time. $\varepsilon_{1-Z} @ 410 \mathrm{~nm}=$ $2200 \mathrm{M}^{-1} \cdot \mathrm{cm}^{-1}$ (for one repeating unit) was used for quantum yield calculations. The photoisomerization quantum yield was calculated to be $5.3 \pm 0.1 \%$ based on three consecutive measurements. 


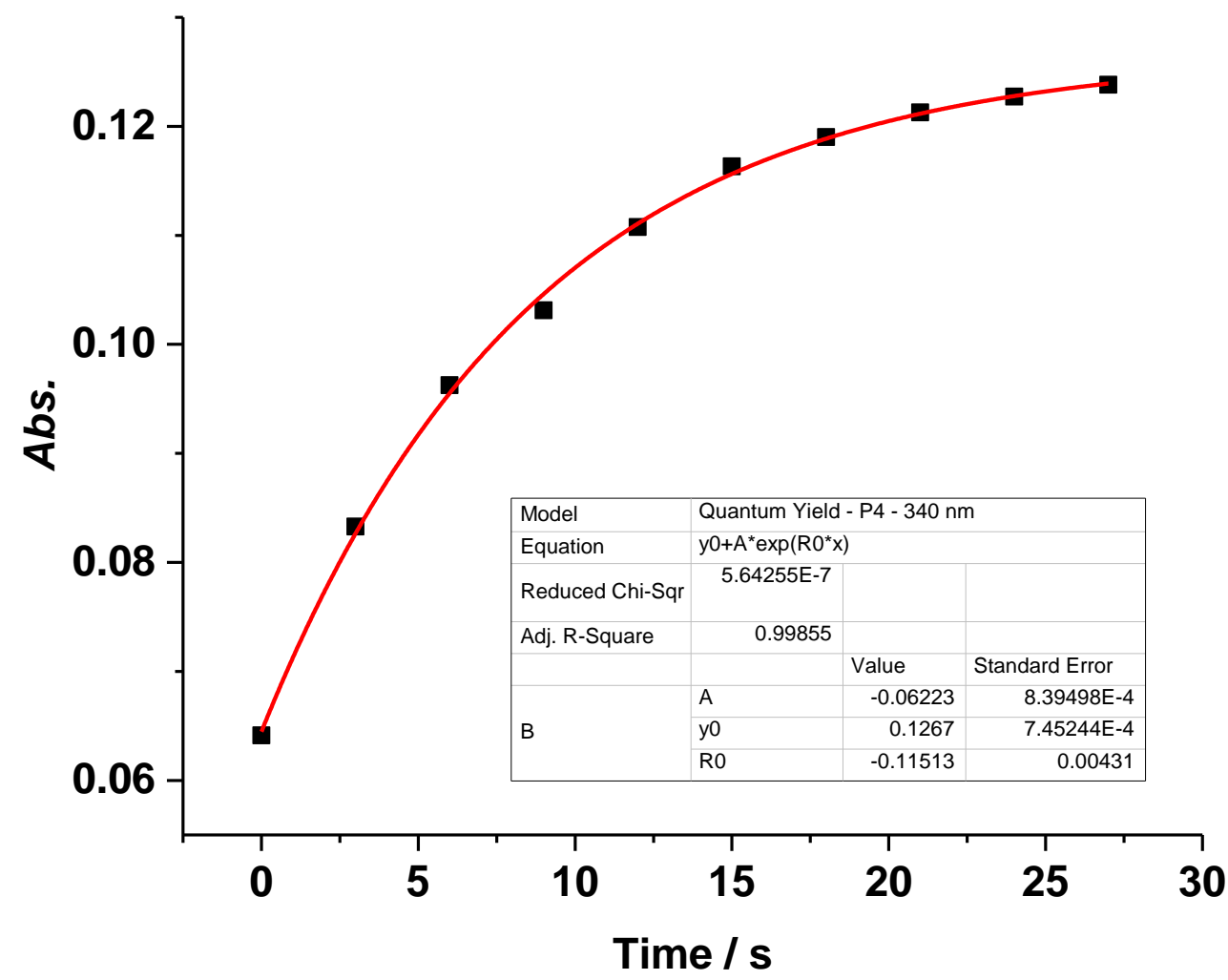

Figure S72. Kinetics for the photoisomerization (irradiation at $340 \mathrm{~nm}$ ) of P4- $E$ to $\mathbf{P 4}-Z$ in DCM $\left(1 \times 10^{-5} \mathrm{M}\right)$ at $298 \mathrm{~K}$; the plot is of absorption $(\lambda=371 \mathrm{~nm})$ as a function of time. $\varepsilon 1-Z @ 340 \mathrm{~nm}=$ $13100 \mathrm{M}^{-1} \cdot \mathrm{cm}^{-1}$ (for one repeating unit) was used for quantum yield calculations. The photoisomerization quantum yield was calculated to be $8.0 \pm 0.8 \%$ based on three consecutive measurements. 


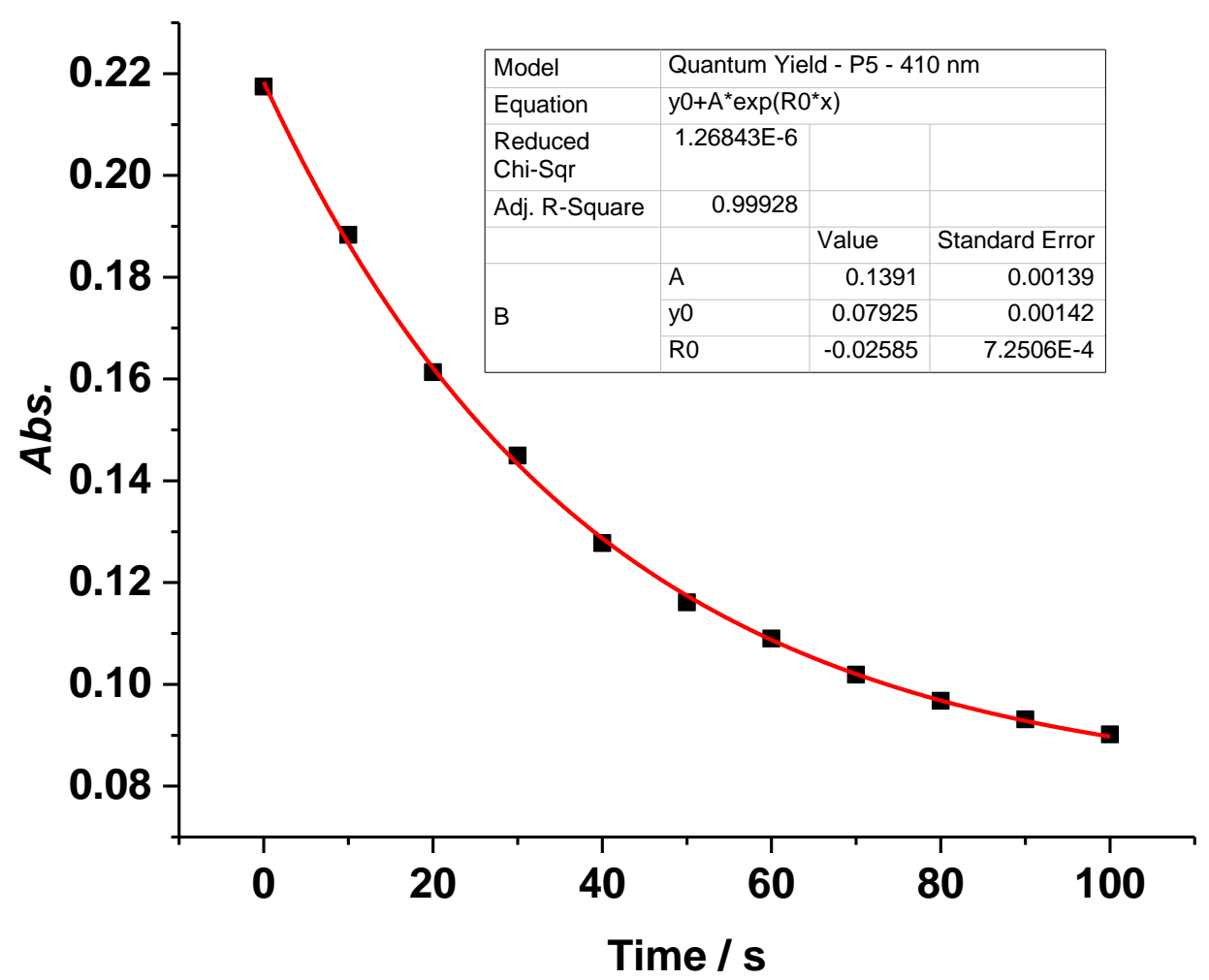

Figure S73. Kinetics for the photoisomerization (irradiation at $410 \mathrm{~nm}$ ) of P5- $Z$ to P5- $E$ in DCM $\left(1 \times 10^{-5} \mathrm{M}\right)$ at $298 \mathrm{~K}$; the plot is of absorption $(\lambda=368 \mathrm{~nm})$ as a function of time. $\varepsilon_{1-Z} @ 410 \mathrm{~nm}=$ $5000 \mathrm{M}^{-1} \cdot \mathrm{cm}^{-1}$ (for one repeating unit) was used for quantum yield calculations. The photoisomerization quantum yield was calculated to be $4.5 \pm 0.2 \%$ based on three consecutive measurements. 


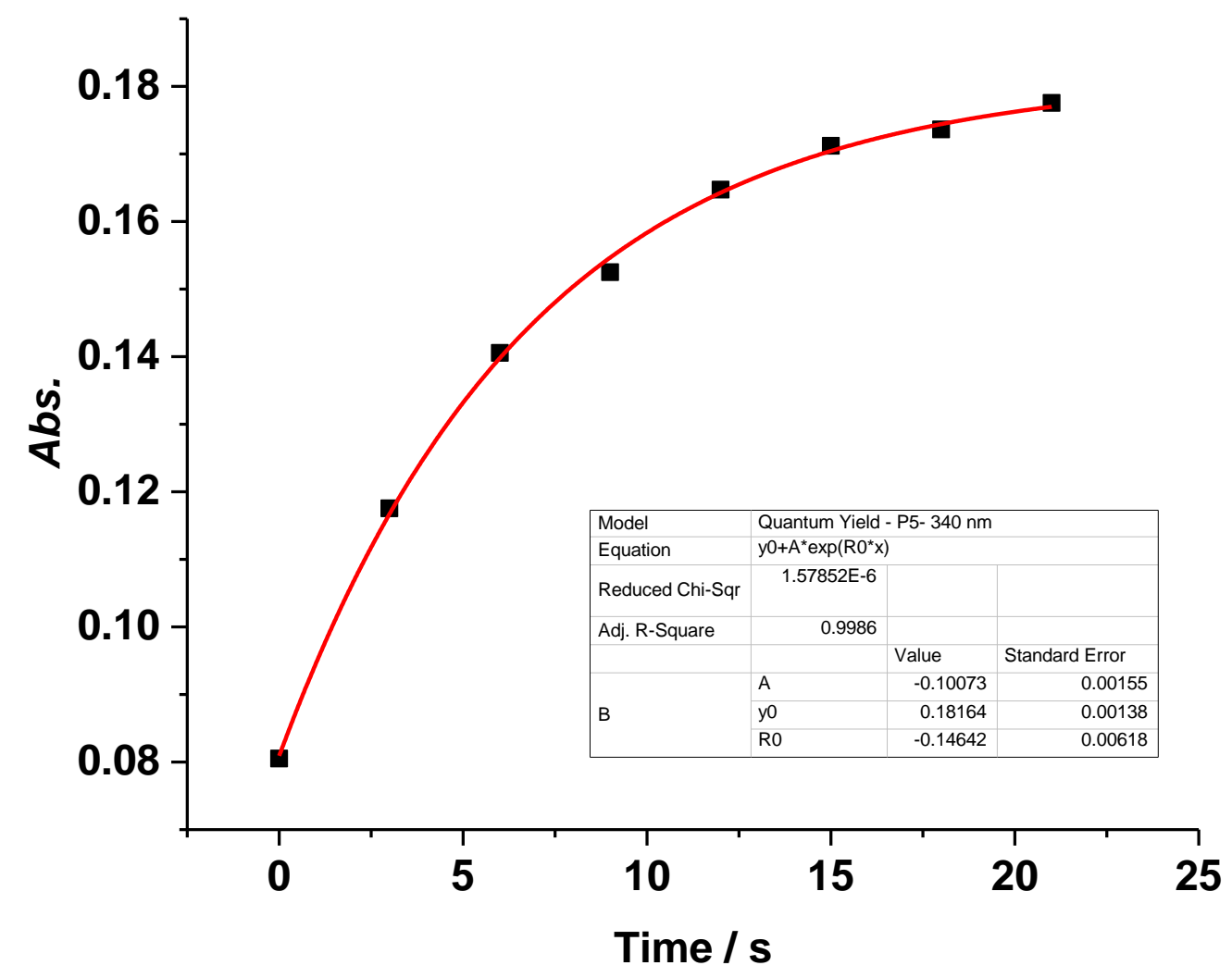

Figure S74. Kinetics for the photoisomerization (irradiation at $340 \mathrm{~nm}$ ) of P5- $E$ to P5- $Z$ in DCM $\left(1 \times 10^{-5} \mathrm{M}\right)$ at $298 \mathrm{~K}$; the plot is of absorption $(\lambda=368 \mathrm{~nm})$ as a function of time. $\varepsilon_{1}-Z @ 340 \mathrm{~nm}=$ $23300 \mathrm{M}^{-1} \cdot \mathrm{cm}^{-1}$ (for one repeating unit) was used for quantum yield calculations. The photoisomerization quantum yield was calculated to be $5.4 \pm 0.1 \%$ based on three consecutive measurements. 


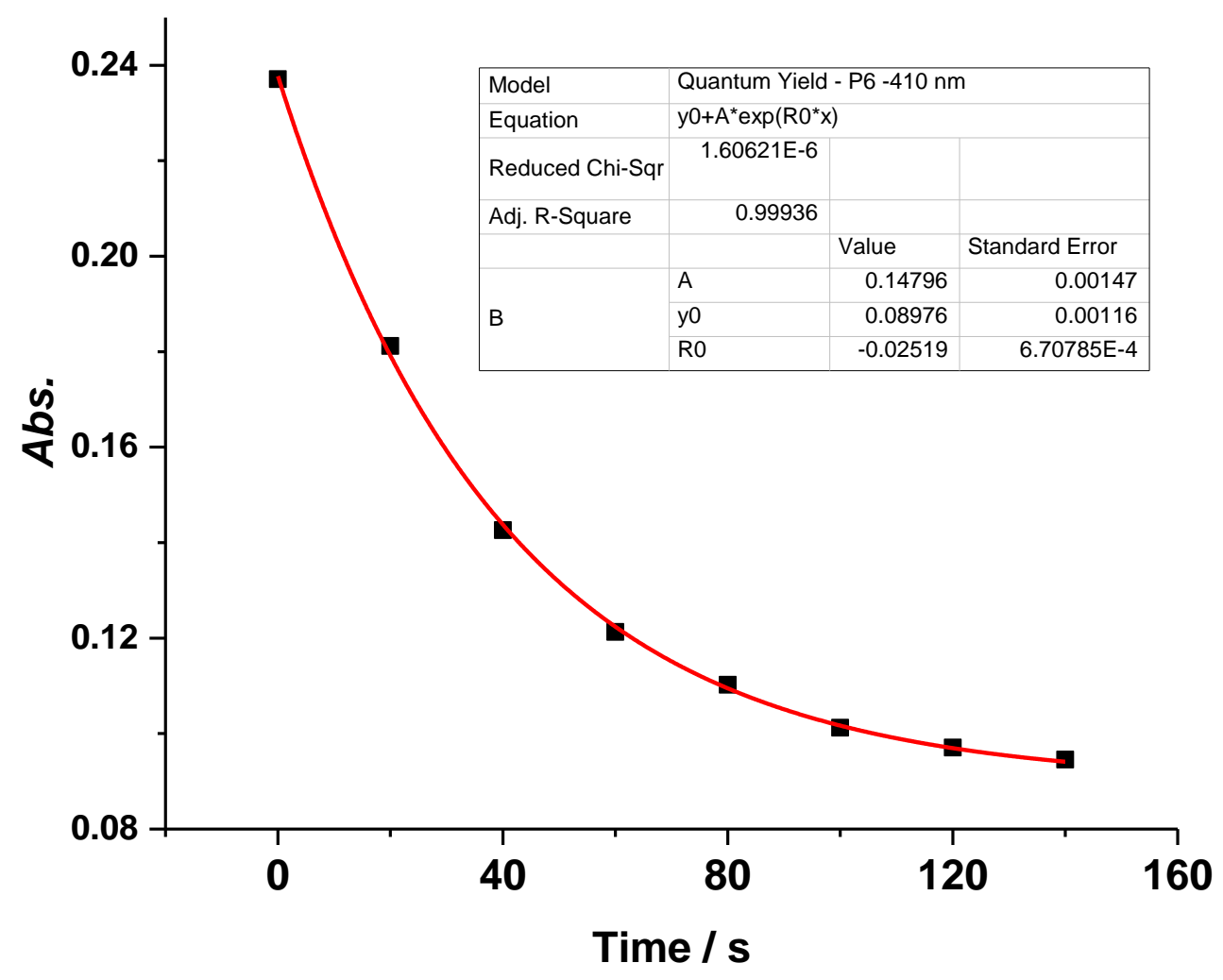

Figure S75. Kinetics for the photoisomerization (irradiation at $410 \mathrm{~nm}$ ) of P6- $Z$ to P6- $E$ in DCM $\left(1 \times 10^{-5} \mathrm{M}\right)$ at $298 \mathrm{~K}$; the plot is of absorption $(\lambda=368 \mathrm{~nm})$ as a function of time. $\varepsilon_{1-Z} @ 410 \mathrm{~nm}=$ $5500 \mathrm{M}^{-1} \cdot \mathrm{cm}^{-1}$ (for one repeating unit) was used for quantum yield calculations. The photoisomerization quantum yield was calculated to be $3.9 \pm 0.4 \%$ based on three consecutive measurements. 


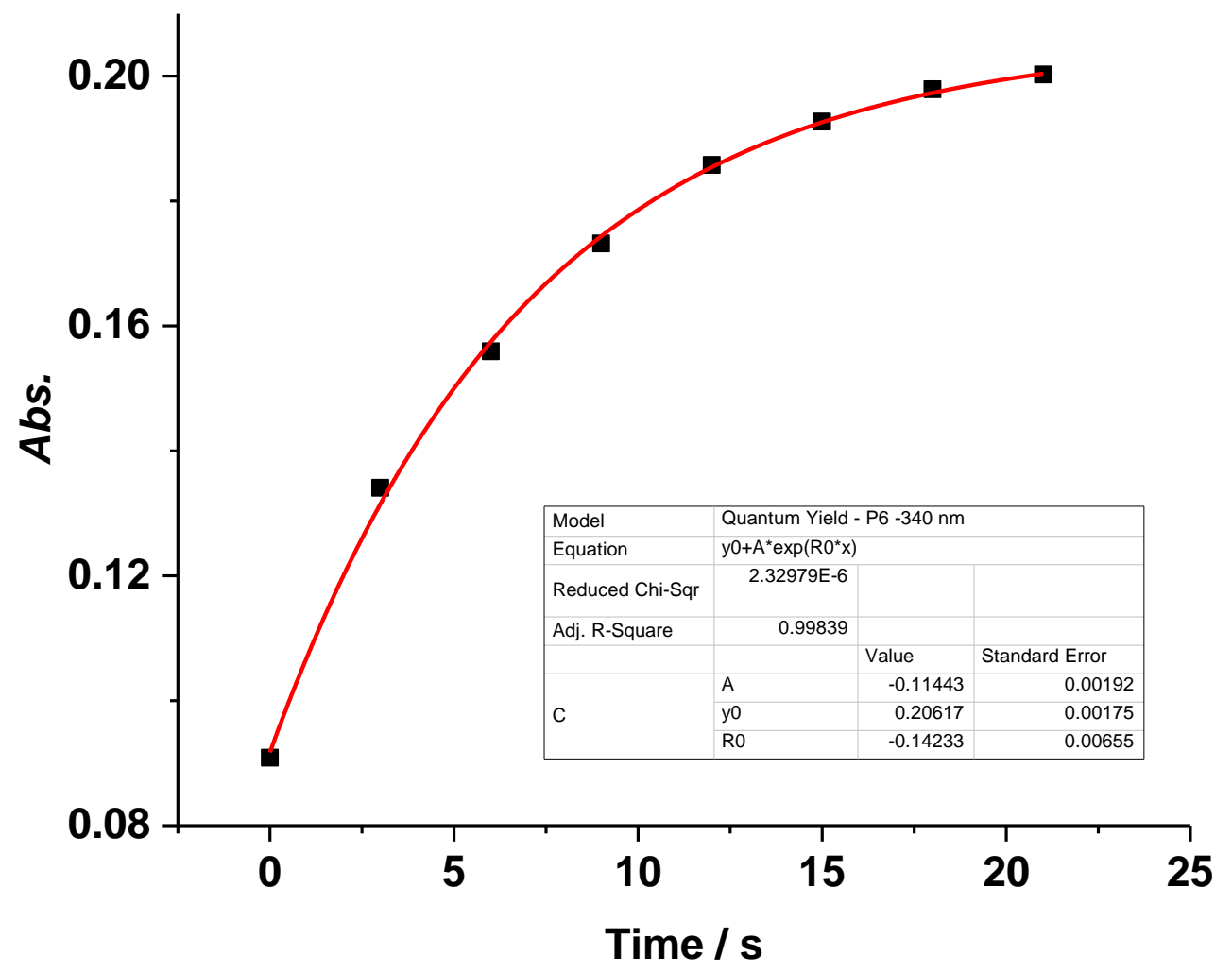

Figure S76. Kinetics for the photoisomerization (irradiation at $340 \mathrm{~nm}$ ) of P6- $E$ to P6- $Z$ in DCM $\left(1 \times 10^{-5} \mathrm{M}\right)$ at $298 \mathrm{~K}$; the plot is of absorption $(\lambda=368 \mathrm{~nm})$ as a function of time. $\varepsilon_{1}-Z @ 340 \mathrm{~nm}=$ $27200 \mathrm{M}^{-1} \cdot \mathrm{cm}^{-1}$ (for one repeating unit) was used for quantum yield calculations. The photoisomerization quantum yield was calculated to be $4.8 \pm 0.5 \%$ based on three consecutive measurements. 


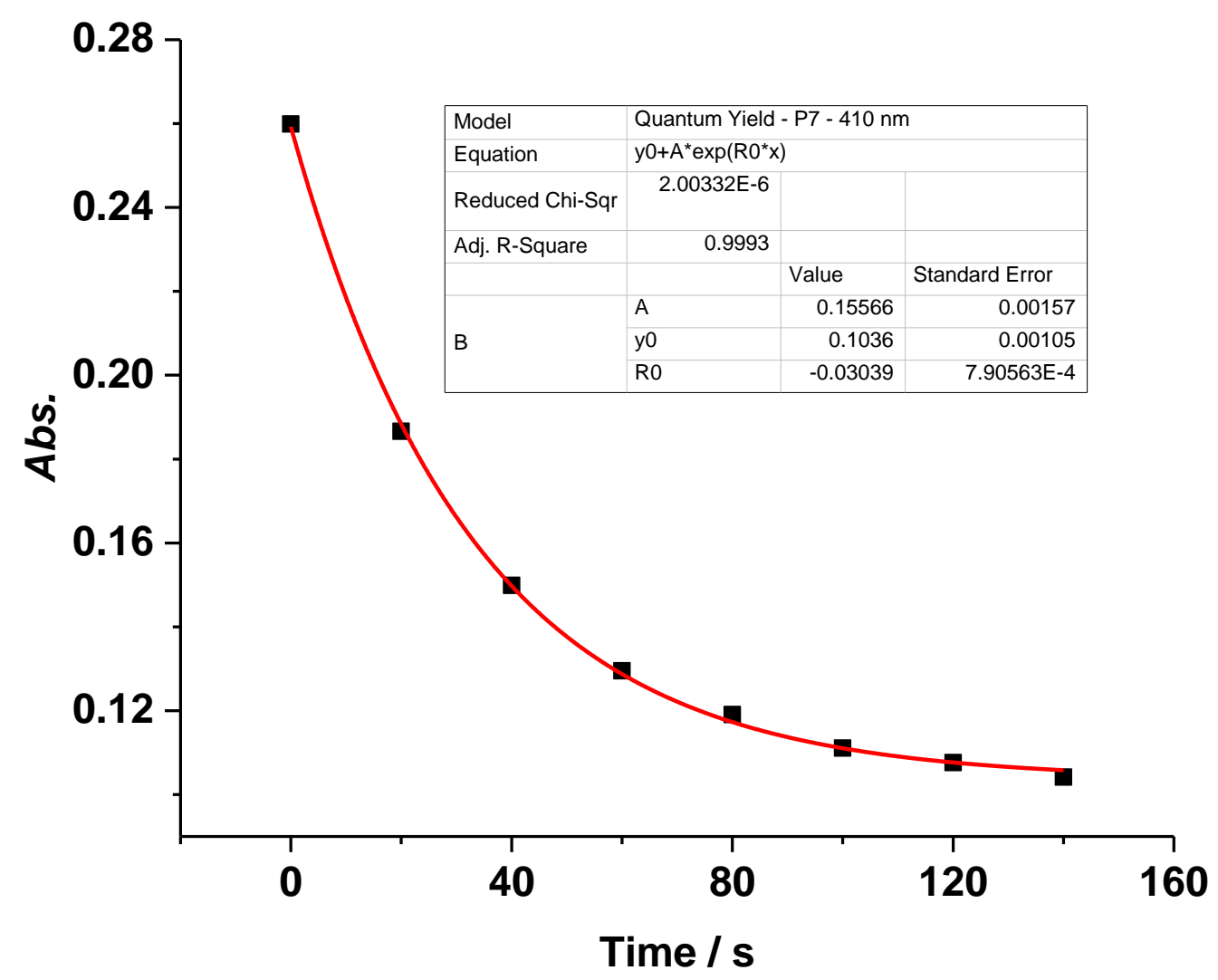

Figure S77. Kinetics for the photoisomerization (irradiation at $410 \mathrm{~nm}$ ) of $\mathbf{P 7}-Z$ to $\mathbf{P 7}-E$ in DCM $\left(1 \times 10^{-5} \mathrm{M}\right)$ at $298 \mathrm{~K}$; the plot is of absorption $(\lambda=368 \mathrm{~nm})$ as a function of time. $\varepsilon 1-Z @ 410 \mathrm{~nm}=$ $6100 \mathrm{M}^{-1} \cdot \mathrm{cm}^{-1}$ (for one repeating unit) was used for quantum yield calculations. The photoisomerization quantum yield was calculated to be $4.2 \pm 0.2 \%$ based on three consecutive measurements. 


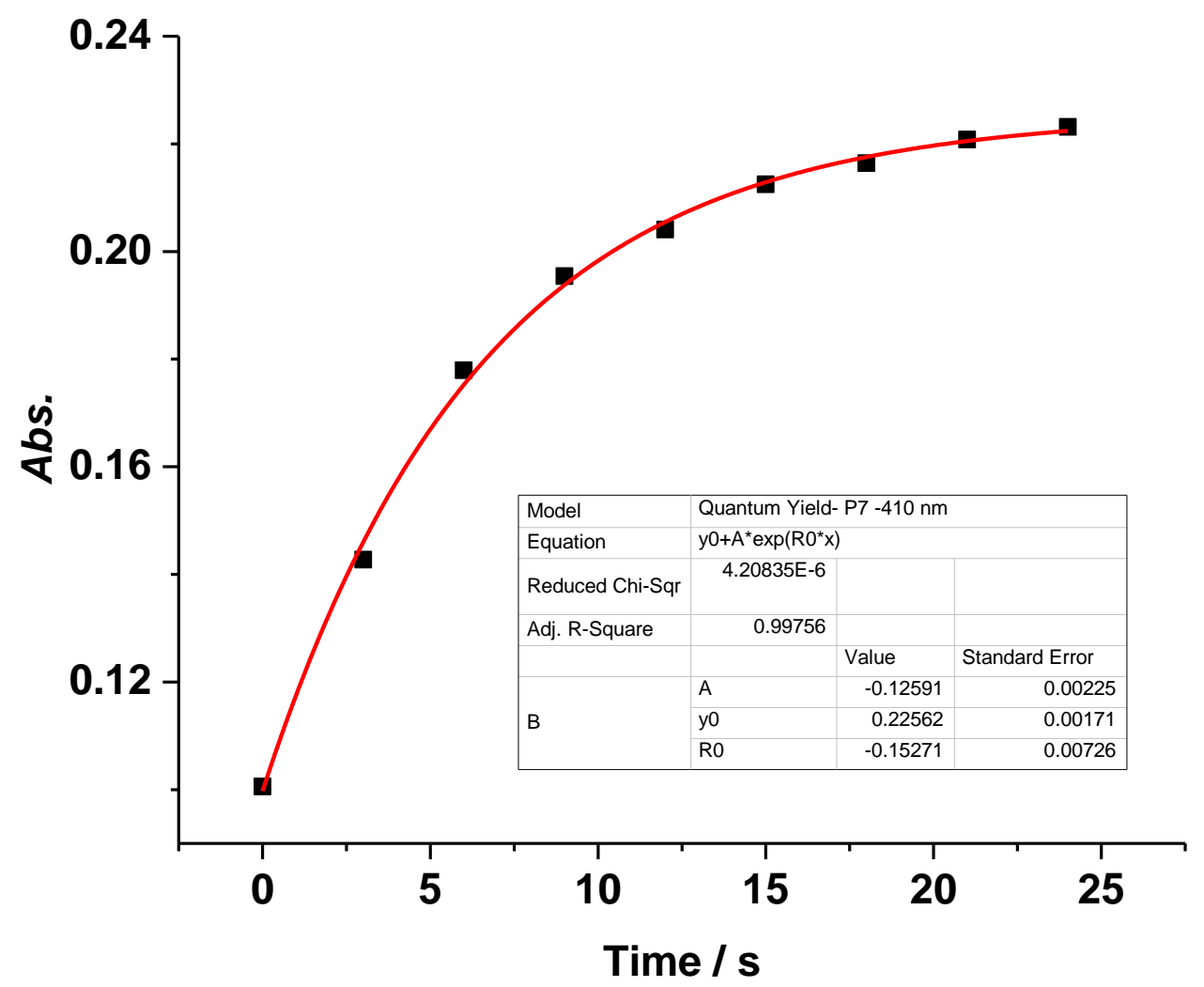

Figure S78. Kinetics for the photoisomerization (irradiation at $340 \mathrm{~nm}$ ) of P7- $E$ to $\mathbf{P 7}-Z$ in DCM $\left(1 \times 10^{-5} \mathrm{M}\right)$ at $298 \mathrm{~K}$; the plot is of absorption $(\lambda=368 \mathrm{~nm})$ as a function of time. $\varepsilon_{1-Z} @ 340 \mathrm{~nm}=$ $30000 \mathrm{M}^{-1} \cdot \mathrm{cm}^{-1}$ (for one repeating unit) was used for quantum yield calculations. The photoisomerization quantum yield was calculated to be $3.8 \pm 0.5 \%$ based on three consecutive measurements. 


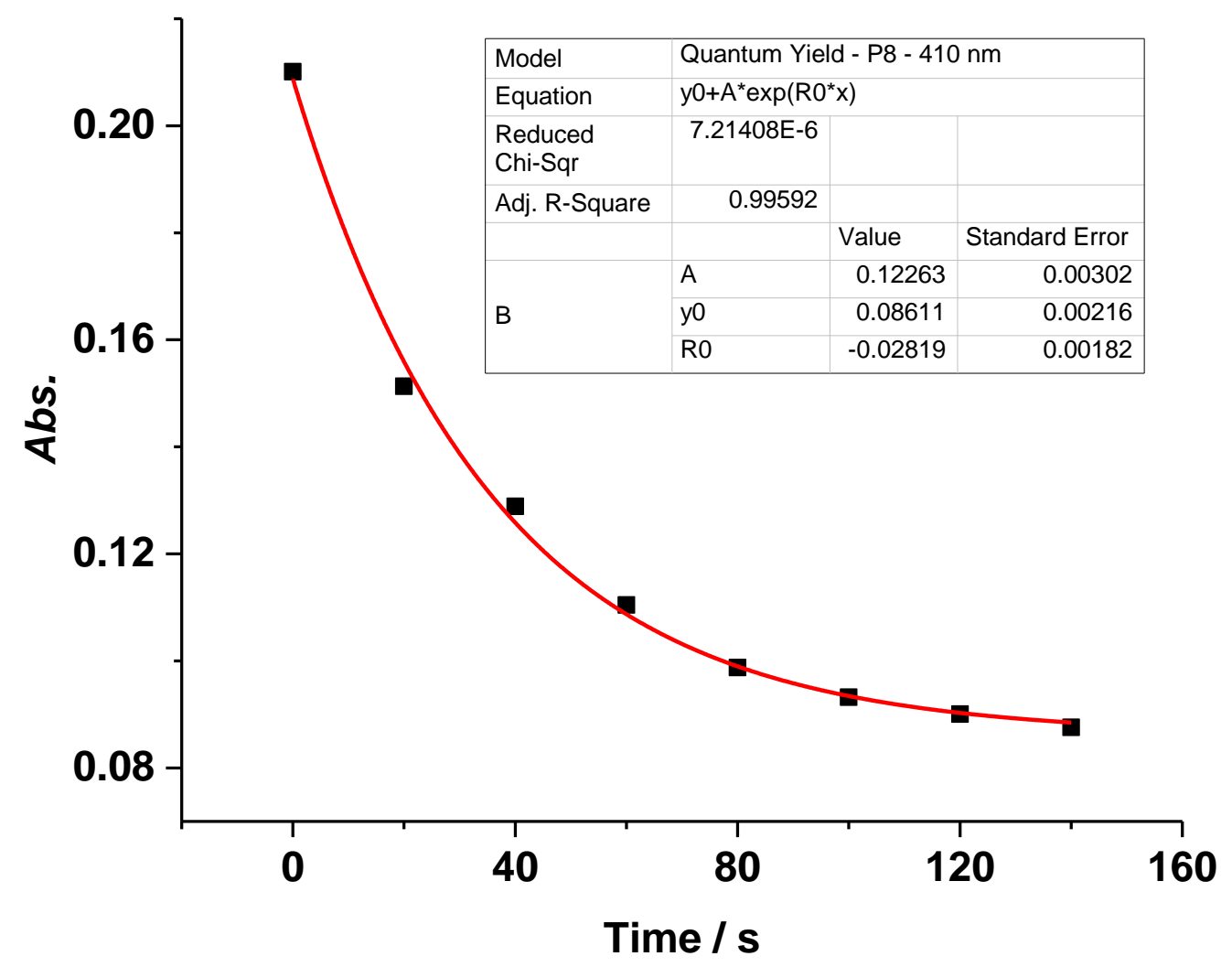

Figure S79. Kinetics for the photoisomerization (irradiation at $410 \mathrm{~nm}$ ) of P8- $Z$ to P8- $E$ in DCM $\left(1 \times 10^{-5} \mathrm{M}\right)$ at $298 \mathrm{~K}$; the plot is of absorption $(\lambda=368 \mathrm{~nm})$ as a function of time. $\varepsilon 1-Z @ 410 \mathrm{~nm}=$ $4600 \mathrm{M}^{-1} \cdot \mathrm{cm}^{-1}$ (for one repeating unit) was used for quantum yield calculations. The photoisomerization quantum yield was calculated to be $5.4 \pm 0.1 \%$ based on three consecutive measurements. 


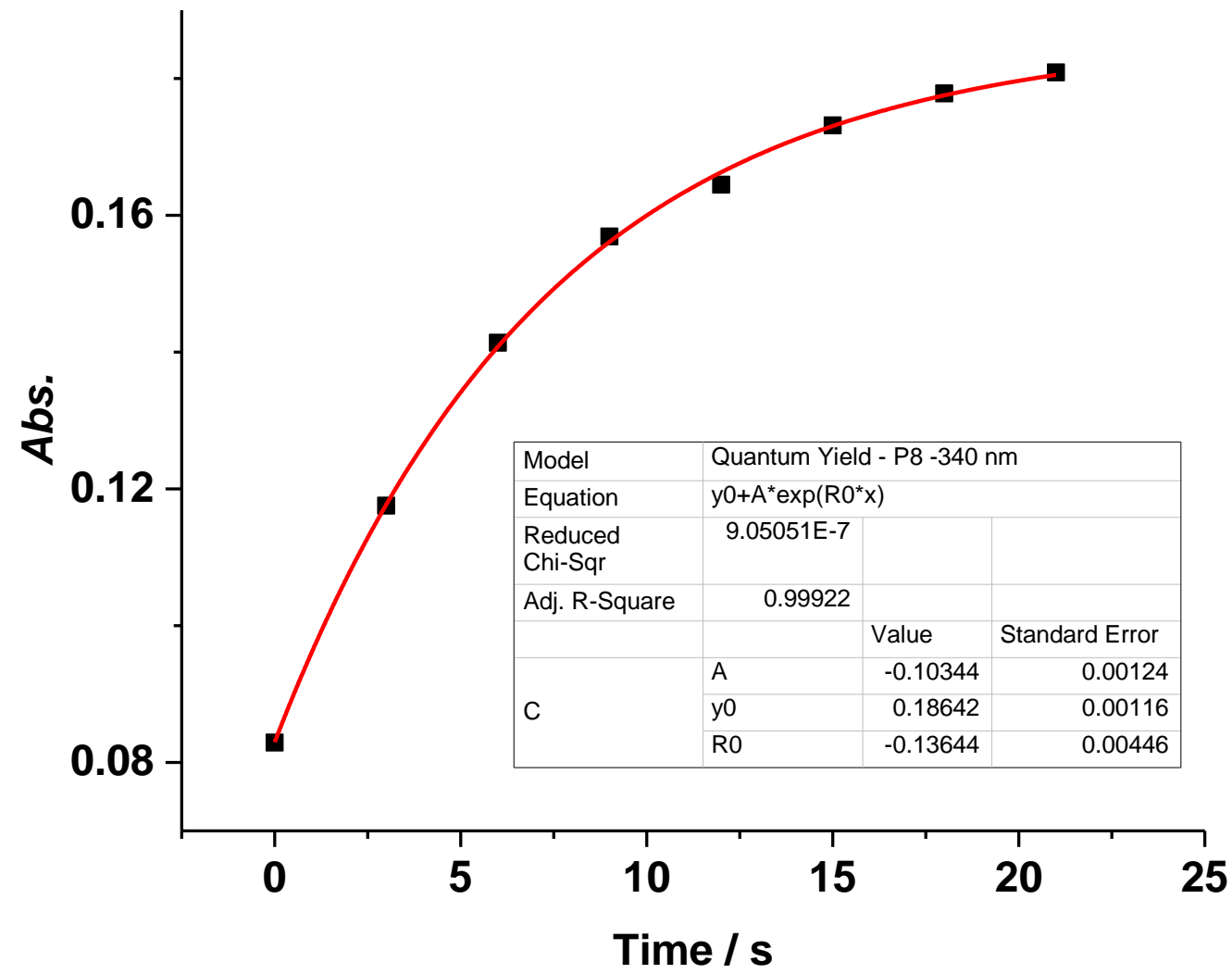

Figure S80. Kinetics for the photoisomerization (irradiation at $340 \mathrm{~nm}$ ) of P8- $E$ to P8- $Z$ in DCM $\left(1 \times 10^{-5} \mathrm{M}\right)$ at $298 \mathrm{~K}$; the plot is of absorption $(\lambda=368 \mathrm{~nm})$ as a function of time. $\varepsilon_{1-Z} @ 340 \mathrm{~nm}=$ $24400 \mathrm{M}^{-1} \cdot \mathrm{cm}^{-1}$ (for one repeating unit) was used for quantum yield calculations. The photoisomerization quantum yield was calculated to be $5.0 \pm 0.8 \%$ based on three consecutive measurements. 


\section{Kinetic Studies}

The model compounds 13-15 were used to estimate the thermal half-lives of the polymers by following a reported procedure. ${ }^{\mathrm{S} 4}$ The thermal half-life of compound $\mathbf{1 3}$ was previously reported. ${ }^{\mathrm{S} 1}$ A solution of 14 or $15\left(1 \times 10^{-3} \mathrm{M}\right)$ in toluene- $d 8$ was prepared and then irradiated at $410 \mathrm{~nm}$ to obtain a $E$ rich solution, which was then heated at $90{ }^{\circ} \mathrm{C}$ under dark. The change in concentration of the $E$ isomer as a function of time was monitored using ${ }^{1} \mathrm{H}$ NMR spectroscopy. This change in isomeric ratio was used to determine the rate of decay (equation 1), which enabled the calculation of the energy barrier for relaxation from $\mathrm{E} \rightarrow \mathrm{Z}$ using the Eyring equation (equation 2). With the activation barrier in hand the Arrhenius equation (equation 3) was used to extrapolate the thermal half-life at room temperature.

$$
k=\frac{\ln \left(\frac{\% E_{0}}{\% E}\right)}{t}
$$

Where $\mathrm{k}$ indicates the rate constant, $\% E_{0}$ indicates the initial percentage of the $E$ isomer, $\% E$ indicates the percentage of the $E$ isomer after incubation and $t$ is the time in seconds.

$$
\Delta G^{\neq}=8.314 \cdot T \cdot\left[23.760+\ln \left(\frac{T}{k}\right)\right]
$$

Where $\Delta \mathrm{G}^{\neq}$indicates the energy barrier to relaxation in $\mathrm{J} \mathrm{mol}^{-1}$ and $T$ is the incubation temperature.

$$
\ln \left(\frac{k_{2}}{k_{1}}\right)=\frac{\Delta G}{R}\left(\frac{1}{T_{1}}-\frac{1}{T_{2}}\right)
$$

Where $\mathrm{k}_{2}$ indicates the rate at the room temperature, $\mathrm{k}_{1}$ indicates the rate at the incubation temperature, $\mathrm{R}$ indicates the ideal gas constant in $\mathrm{J} \mathrm{K}^{-1} \mathrm{~mol}^{-1}, \mathrm{~T}_{2}$ is $298 \mathrm{~K}$, and $\mathrm{T}_{1}$ indicates the incubation temperature in kelvin.

Table S2. The rate of the $E \rightarrow Z$ isomerization rate, energy barrier and thermal half-life at $298 \mathrm{~K}$.

\begin{tabular}{cccc}
\hline Hydrazone & $k\left(\mathrm{~s}^{-1}\right)^{\mathrm{a}}$ & $\Delta \mathrm{G}^{\neq}\left(\mathrm{kcal} \mathrm{mol}^{-1}\right)^{\mathrm{a}}$ & $\tau_{1 / 2}($ years $)$ \\
\hline $\mathbf{1 3}^{\mathrm{b}}$ & $(4.1 \pm 0.1) \mathrm{E}-12^{\mathrm{b}}$ & $32.9 \pm 0.1^{\mathrm{b}}$ & $5357 \pm 101^{\mathrm{b}}$ \\
& & & \\
$\mathbf{1 4}$ & $(5.9 \pm 0.4) \mathrm{E}-11$ & $31.5 \pm 0.5$ & $378 \pm 30$ \\
& & & \\
$\mathbf{1 5}$ & $(2.6 \pm 0.1) \mathrm{E}-11$ & $31.9 \pm 0.1$ & $837 \pm 13$
\end{tabular}

a. Average of three measurements. $b$. Taken from ref S1 and measured in $p$-xylene 
10. Summary of Glass Transition Temperature Measurements for the HydrazoneContaining Polymers

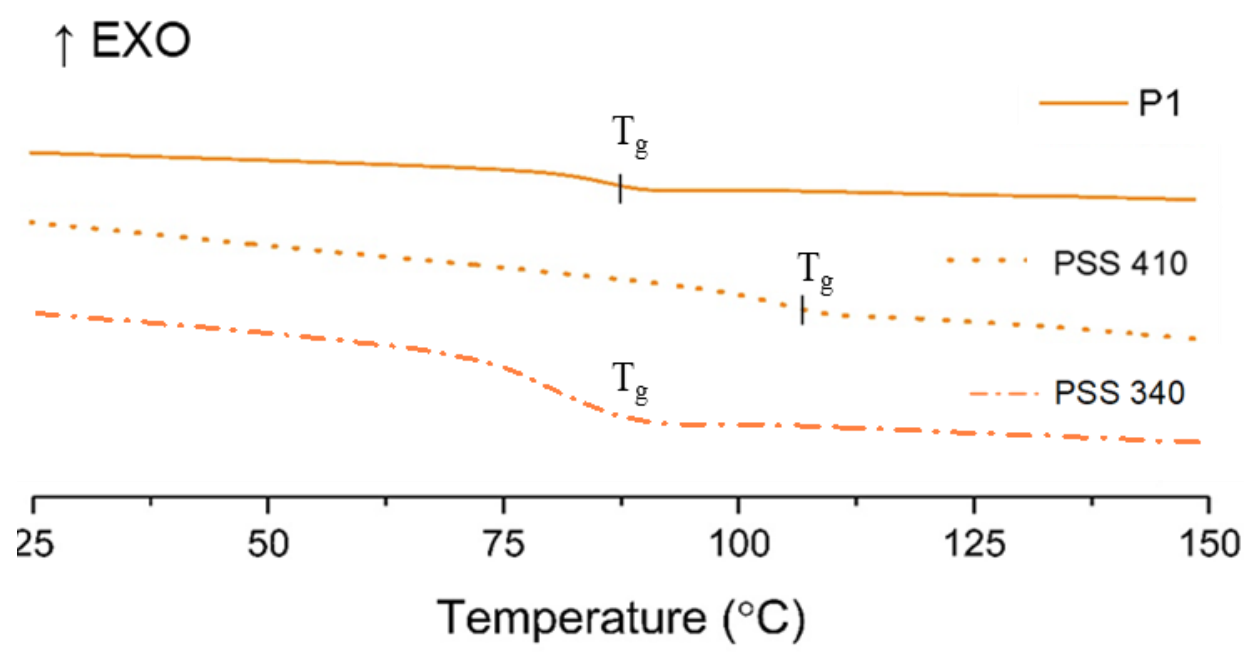

Figure S81. DSC Curves of pristine P1 ( $Z$ configuration), and sample after 410 and $340 \mathrm{~nm}$ irradiation.

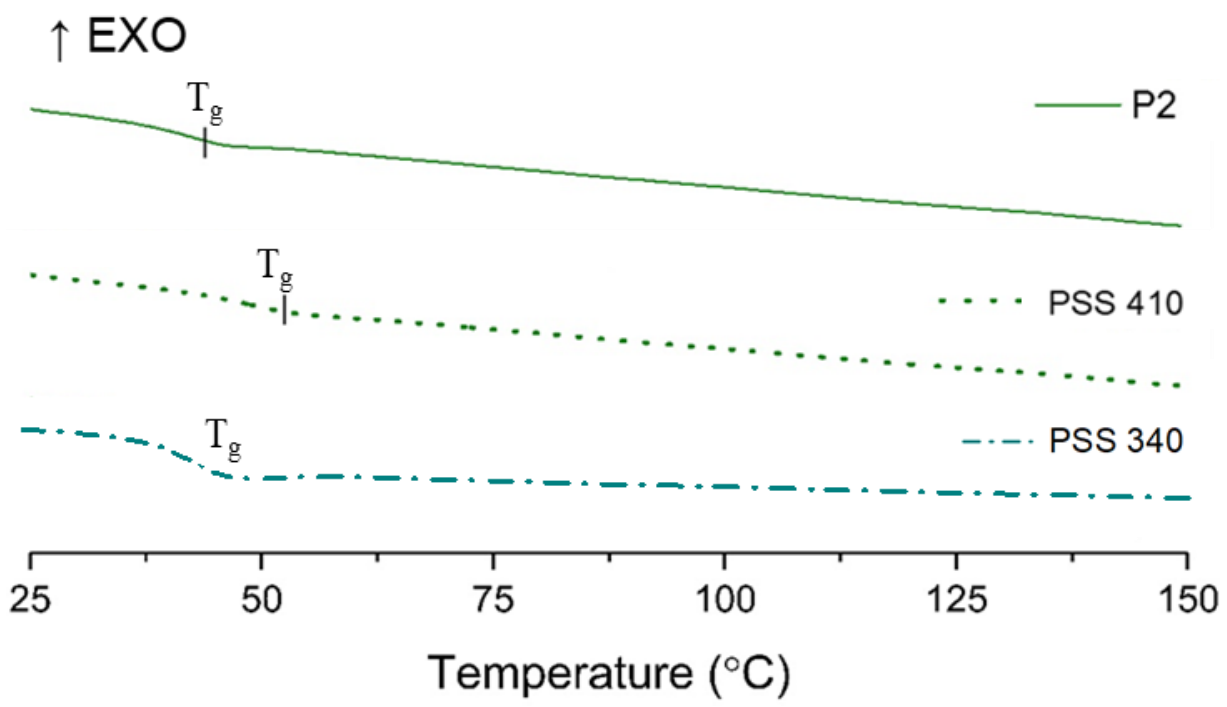

Figure S82. DSC Curves of pristine P2 ( $Z$ configuration), and sample after 410 and $340 \mathrm{~nm}$ irradiation. 


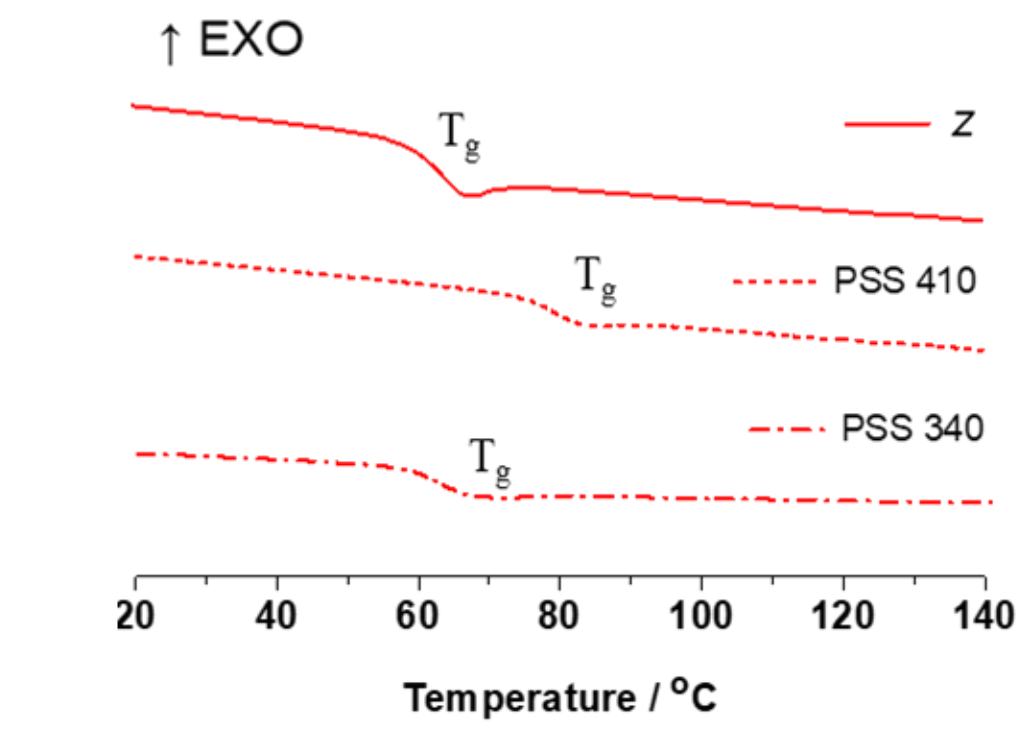

Figure S83. DSC Curves of pristine P3 ( $Z$ configuration), and sample after 410 and $340 \mathrm{~nm}$ irradiation

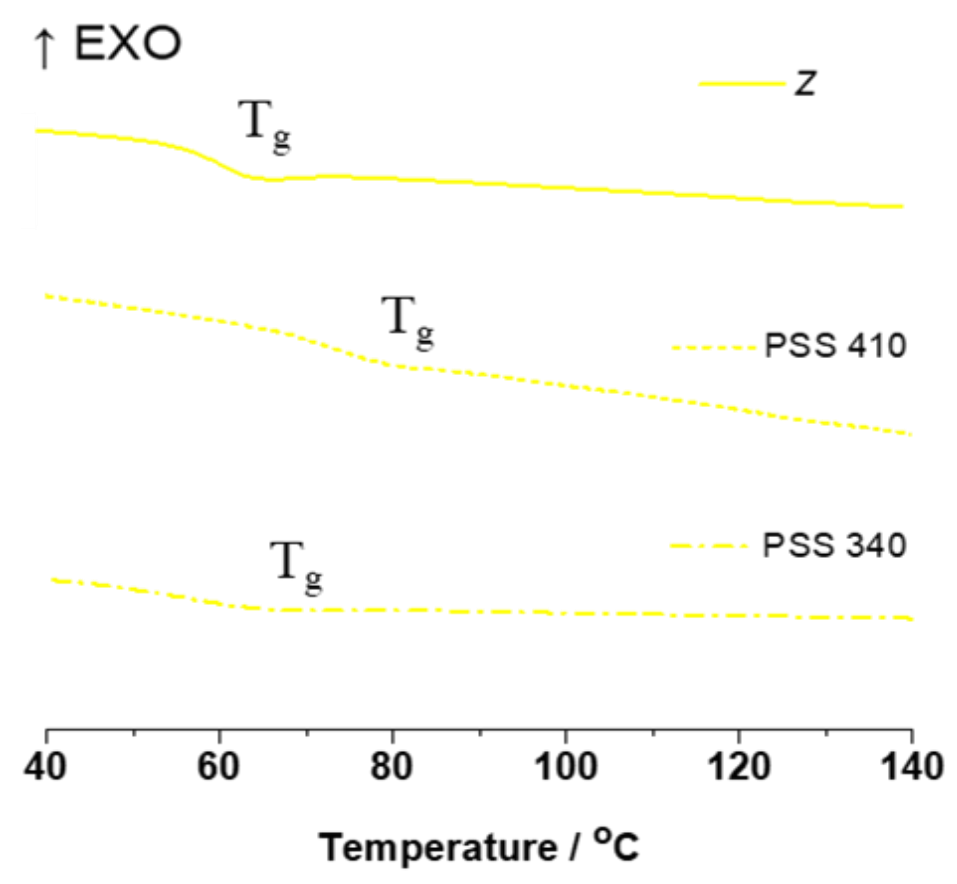

Figure S84. DSC Curves of pristine P4 ( $Z$ configuration), and sample after 410 and $340 \mathrm{~nm}$ irradiation 


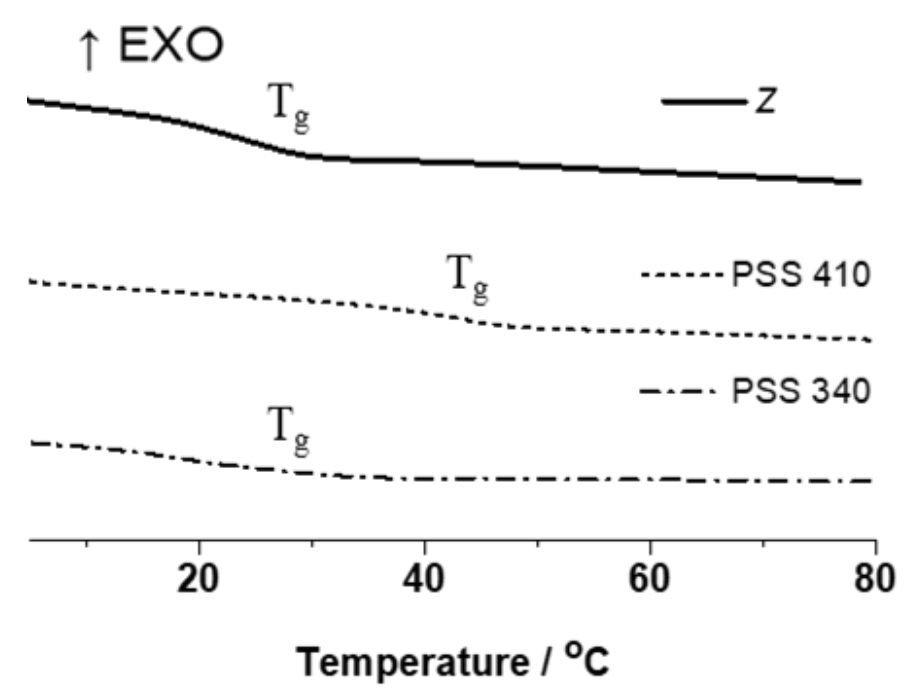

Figure S85. DSC Curves of pristine P6 ( $Z$ configuration), and sample after 410 and $340 \mathrm{~nm}$ irradiation

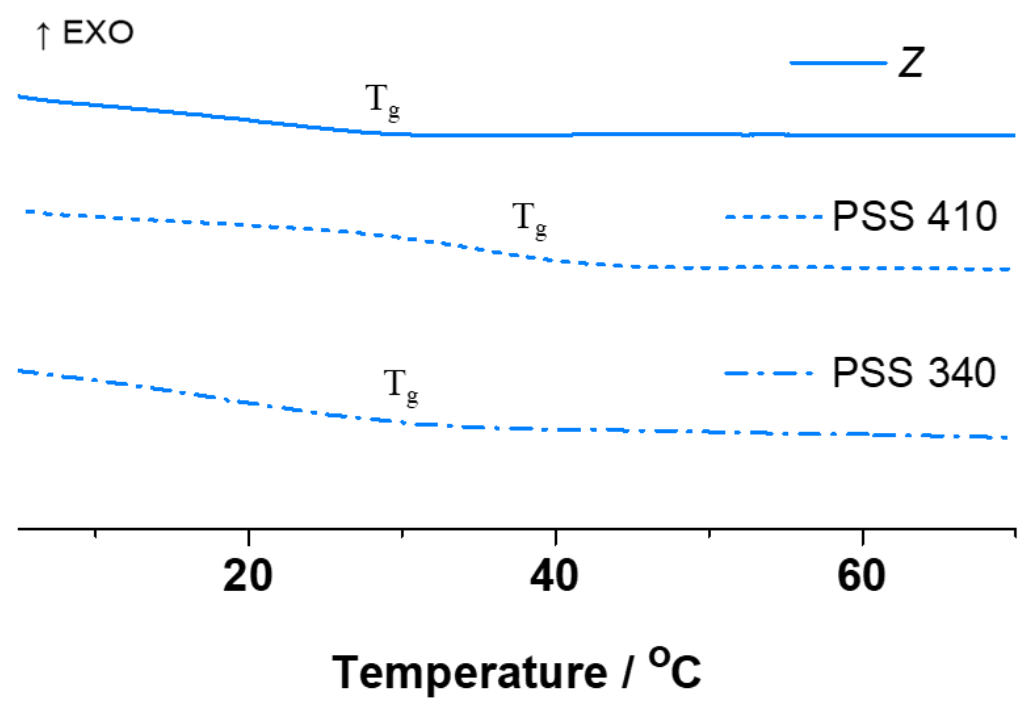

Figure S86. DSC Curves of pristine $\mathbf{P 7}$ ( $Z$ configuration) and after 410 and $340 \mathrm{~nm}$ irradiation 


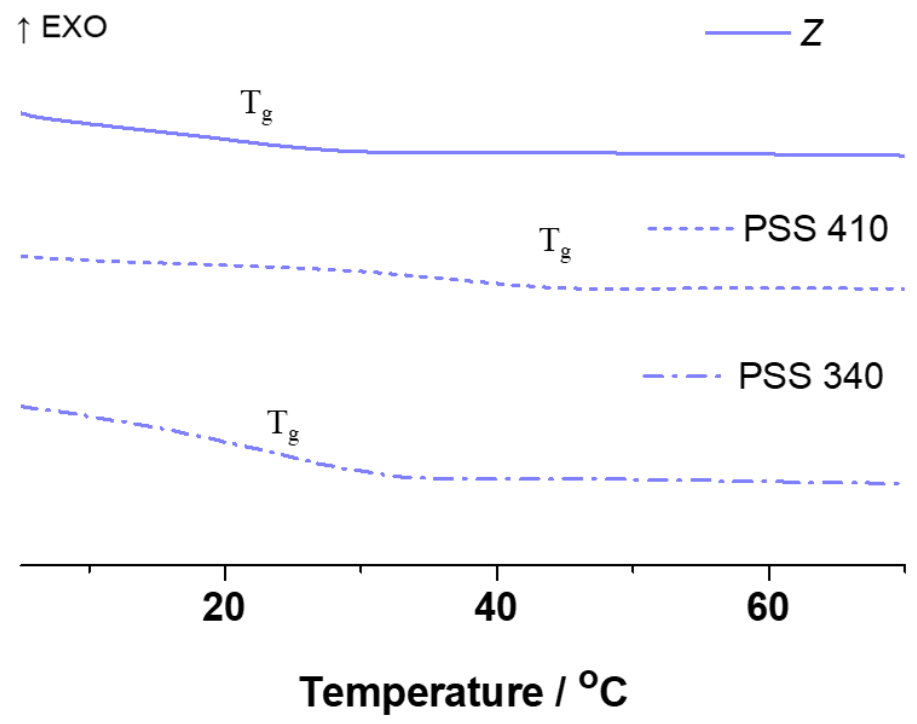

Figure S87. DSC Curves of pristine P8 ( $Z$ configuration), and sample after 410 and $340 \mathrm{~nm}$ irradiation 


\section{Nanoindentation for photo-stiffening verification}

Two samples of $\mathbf{P 5}$ were prepared by hot stamping fabrication at $130{ }^{\circ} \mathrm{C}$, resulting in approximately $30 \mu \mathrm{m}$ thick polymer films on top of $1 \mathrm{~cm} \times 1 \mathrm{~cm} \times 500 \mu \mathrm{m}$ silicon substrates. One was kept in the dark as pristine sample, while the other was irradiated with $410 \mathrm{~nm}\left(600 \mathrm{~mW} / \mathrm{cm}^{2}\right)$ for one hour, to maximize the $E$ isomer content. Nanoindentation measurements on both the P5 polymer samples (pristine and light irradiated conditions) were performed using an Alemnis insitu nanoindenter (Alemnis AG, Switzerland) integrated inside a Zeiss Leo Scanning Electron Microscope (SEM). The samples were tested from room temperature to elevated temperatures close to their respective glass transition temperatures. A diamond Berkovich tip was used for the indentation measurements. The maximum indentation depth was less than $2 \mu \mathrm{m}(<10 \%$ film thickness) to avoid elastic influence from the silicon substrate. Compliance and tip area calibrations were performed on fused silica and ultrafine-grained aluminum calibration samples prior to the tests.

The indentation profile comprised of the following segments: (i) displacement controlled, linear loading ramp of $10 \mu \mathrm{m} / \mathrm{s}$ till a load target of $0.5 \mathrm{mN}$ is reached; (ii) displacement controlled, linear loading ramp of $10 \mu \mathrm{m} / \mathrm{s}$ by additional $1.2 \mu \mathrm{m}$ (from the start of this segment); (iii) load controlled, constant load hold segment for 1s; and (iv) displacement controlled, linear unloading to $0 \mu \mathrm{m}$ at $10 \mu \mathrm{m} / \mathrm{s}$. Except for the peak load hold segment, all other indentation segments are displacement controlled. Indentation duration was kept less than $1.5 \mathrm{~s}$ to minimize the influence of thermal drift, an artefact of elevated temperature nanoindentation measurements. A minimum of 10 indents were performed at each temperature. Load-displacement data from the indents were analyzed using Oliver \& Pharr analysis ${ }^{\mathrm{S} 5}$ to calculate indentation modulus and hardness.

The Alemnis experimental set-up used for elevated temperature nanoindentation measurements is shown in Figure S79a. The tip and the sample were heated independently using miniaturized heaters. Thermocouples were mechanically clamped to the sample stub and the tip holder to separately monitor their temperatures. The indenter frame was water cooled to stabilize frame drift. The heating power was locked (i.e., kept constant) during indentation measurements to avoid thermal fluctuations from influencing load-displacement data. The sample was mechanically clamped on a copper sample holder for the elevated temperature measurements (Figure S79b). Indentation measurements were performed in increments of $5-10{ }^{\circ} \mathrm{C}$ up to the highest temperature possible, beyond which the polymer starts sticking to the tip due to excessive 
thermal softening and precludes any meaningful mechanical measurements.

a)

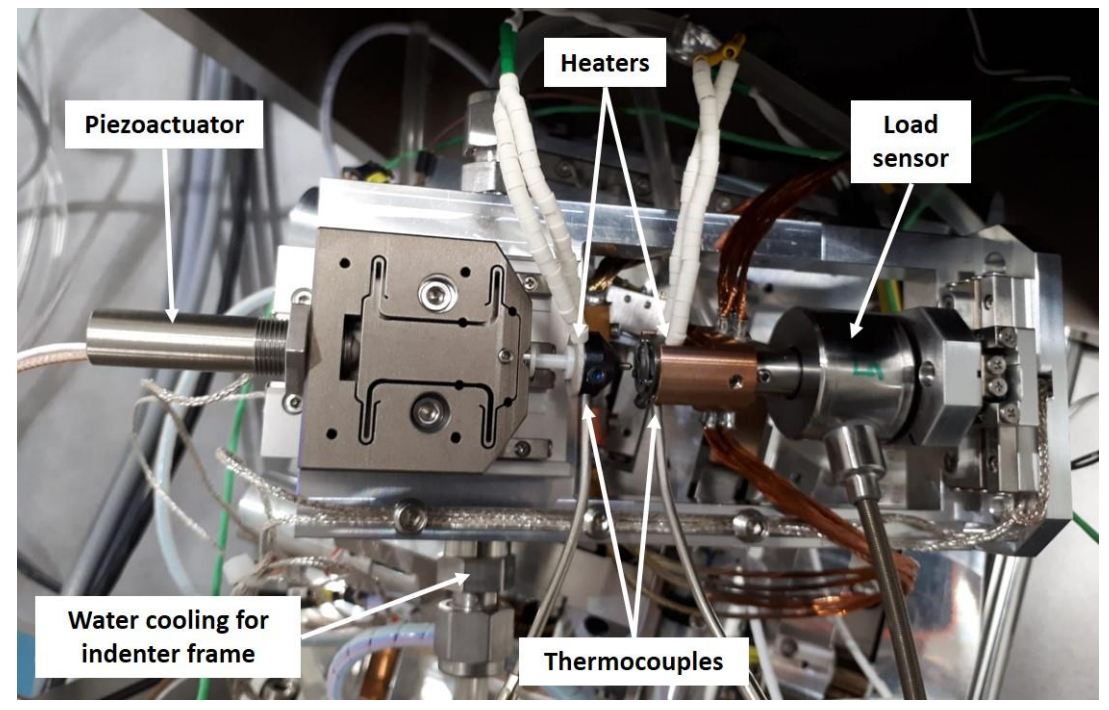

b)

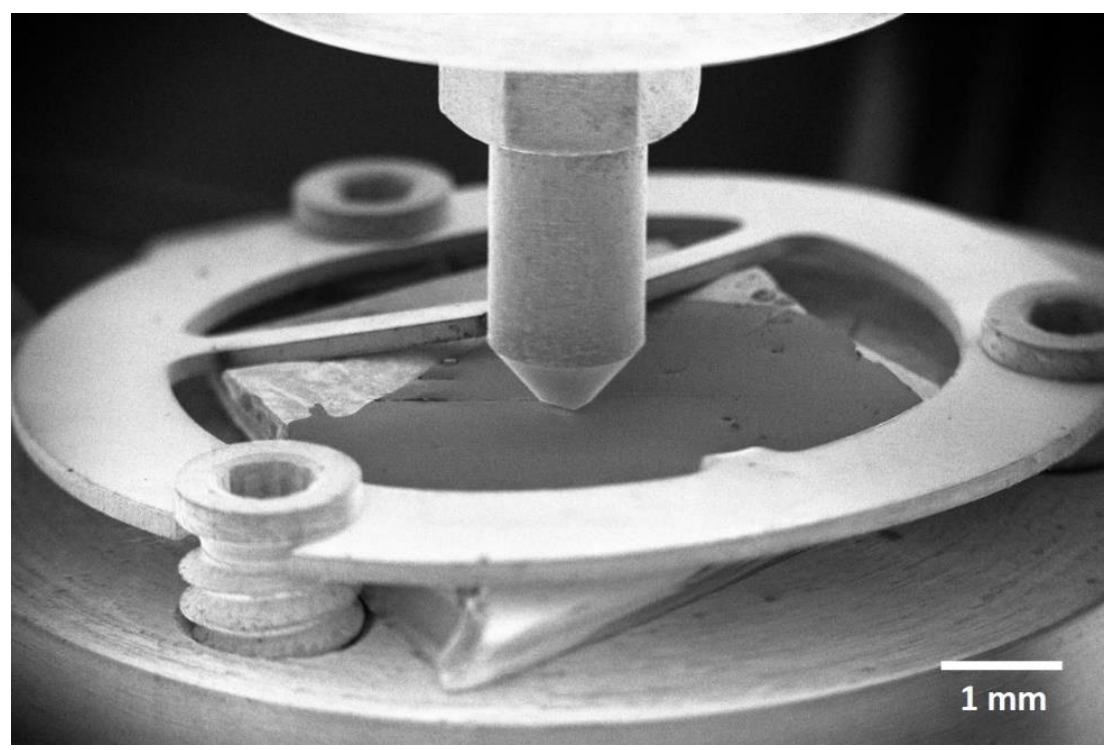

Figure S88 a) Alemnis in-situ SEM nanoindenter set-up for elevated temperature nanoindentation studies utilizing independent heaters and thermocouples for both the tip and the sample, and b) SEM image of the indenter tip and the $30 \mu \mathrm{m}$ thick P5 polymer film deposited on silicon substrate prior to indentation. The mechanical clamping of the sample to the copper stub below can be seen as well. 
The protocol used to minimize thermal drift is briefly described below. The established elevated temperature nanoindentation protocols for isothermal contact and reduction of thermal drift rates to less than $10 \mathrm{~nm} / \mathrm{min}^{\mathrm{S} 6, \mathrm{S7}}$ do not work well on polymers due to low thermal conductivity and viscoelastic behavior. A slightly different approach was adopted. The tip apex temperature was calibrated by performing Berkovich indents on a polished copper stub, to which a thermocouple was mechanically clamped for accurate temperature readout. The tip temperature was iteratively varied, while keeping the copper stub temperature constant, to determine the corresponding tip temperature for $\sim$ zero displacement drift rate. ${ }^{\mathrm{S} 6 \mathrm{S7}}$ Since zero drift is obtained at iso-thermal contact only, the tip apex temperature can be considered equal to the copper stub temperature (measured by the sample-side thermocouple). This allows calibrating the temperature gradient between the tip apex and the location where the tip-side thermocouple is fixed (Figure S80). The tip apex temperature was found to be slightly higher than the tip-side thermocouple readout temperature.

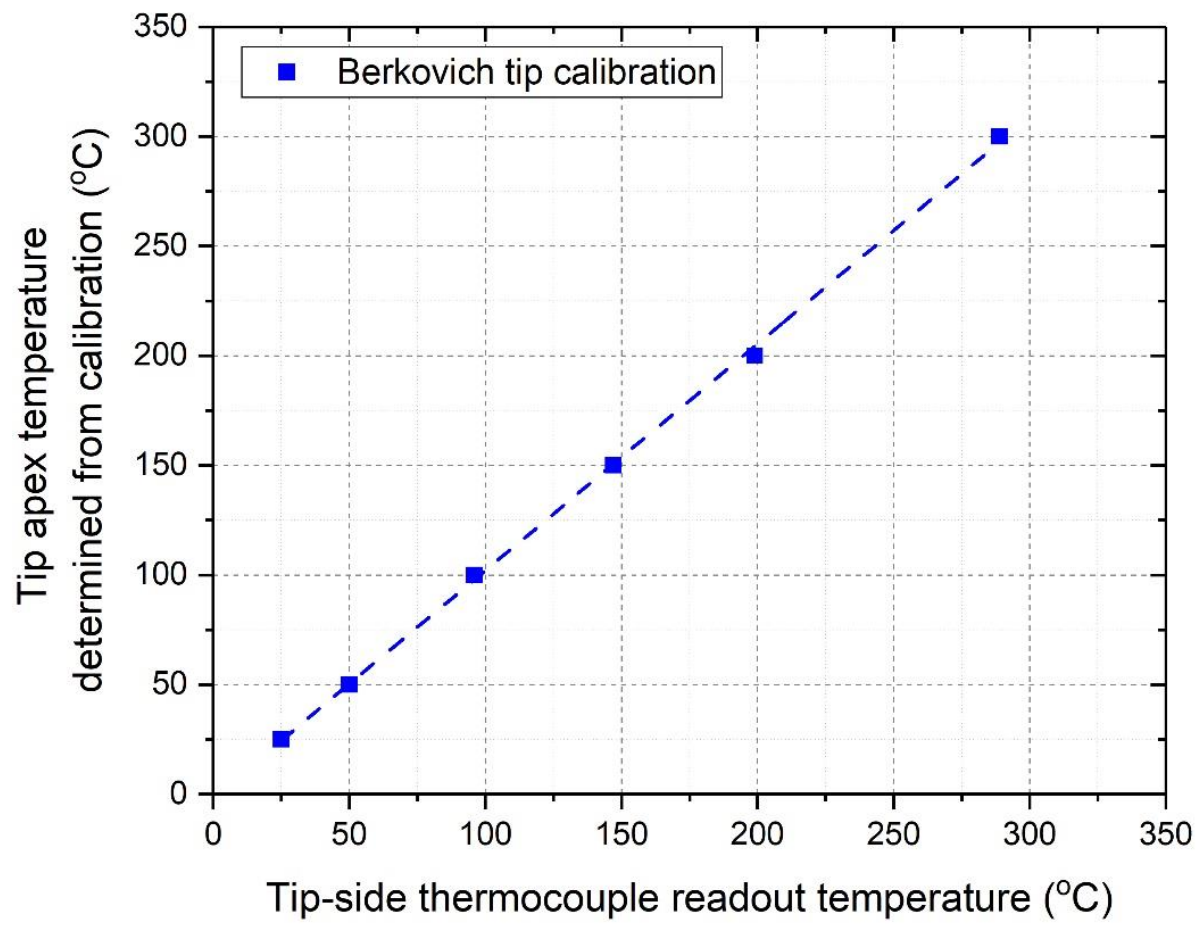

Figure S89. Thermal calibration correlating the tip apex temperature with the tip-side thermocouple readout temperature. 
a)

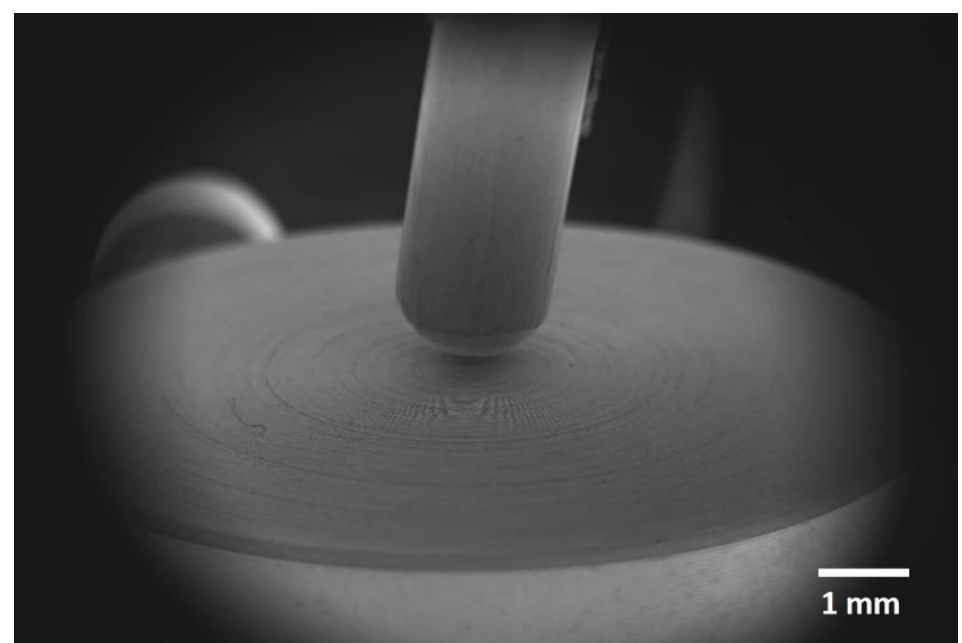

b)

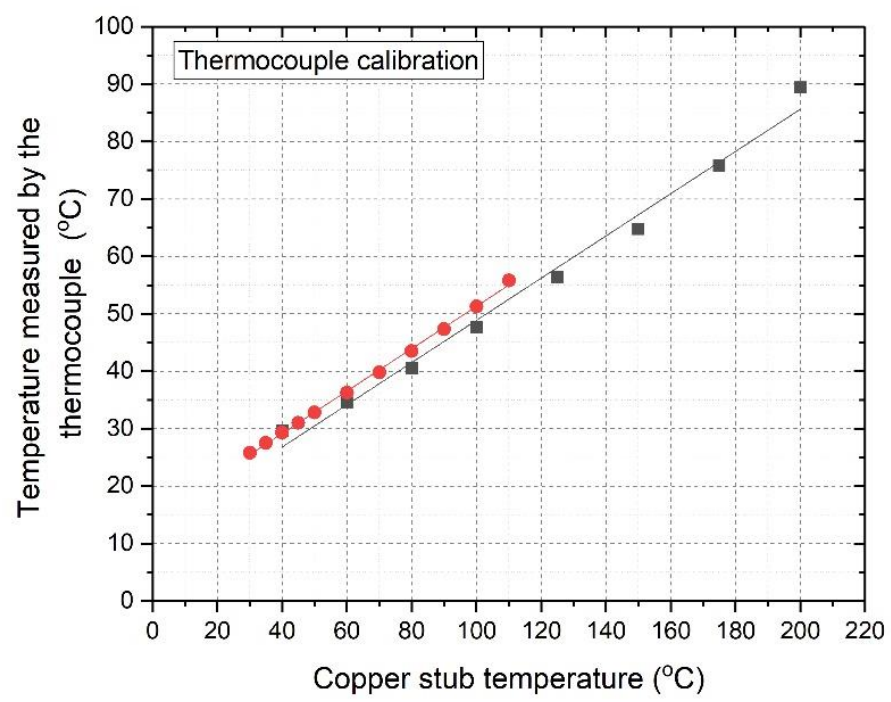

Figure S90 a) SEM image showing the calibration of the thermocouple by bringing it in contact with the heated copper stub, and b) Thermocouple calibration data shows $50 \%$ difference between the actual surface temperature of the copper stub and the temperature readout by the thermocouple when brought in contact. Two repeats suggest a temperature measurement accuracy within $10{ }^{\circ} \mathrm{C}$.

Thereafter, attempts were made to measure the polymer surface temperature directly using a thermocouple. However, measuring the actual surface temperature of a $30 \mu \mathrm{m}$ thick polymer film is relatively difficult. The thermocouple used for these experiments has a larger thermal mass in comparison to the thin polymer film. Thus, the thermocouple acts as a heat-sink when brought in contact with the polymer film and most likely results in localized temperature drop in the polymer film itself. In addition, the light irradiated P5 sample may undergo exothermic reaction when 
maintained at elevated temperatures for prolonged times, due to thermal $E \rightarrow Z$ isomerization of a fraction of the hydrazone pendants, which makes the calibration non-linear and introduces further complexity in experimental determination of polymer surface temperature. To get around this problem, the surface temperature of the Si substrate was measured instead. This provides consistent temperature readings to compare both P5 samples (pristine and light-irradiated conditions). First, the thermocouple itself was calibrated on the copper stub, heated to various temperatures, to determine the offset between the temperature readout by the thermocouple and the actual temperature of the copper stub. This procedure comprised of using the thermocouple itself to apply a load of $100 \mathrm{mN}$ on to the copper stub (Figure S81a) and holding the load constant until the thermocouple temperature readout stabilized. Calibration curves were generated correlating the thermocouple temperature measured temperature with the actual temperature of the copper stub (Figre S81b). The measurement accuracy of the $\mathrm{Cu}$ stub surface temperature was found to be within $10^{\circ} \mathrm{C}$ based on multiple measurements. Thereafter, the calibrated thermocouple was used to measure the surface temperature of the Si wafer (Figure S82).

Based on these calibrations, the tip and the polymer samples were heated such that the tip apex temperature matches with the silicon surface temperature. This provided a near iso-thermal contact condition for performing nanoindentation measurements at elevated temperatures. In addition, keeping the indentation duration to less than $1.5 \mathrm{~s}$ further minimizes thermal drift. The overall displacement drift in the reported measurements is estimated to not exceed $10 \mathrm{~nm}$ for indentation depths of $2 \mu \mathrm{m}(<1 \%$ of displacement data). Silicon surface temperature is reported when discussing the elevated temperature results, e.g. hardness and indentation modulus, on the P5 polymer samples in the manuscript. 
a)

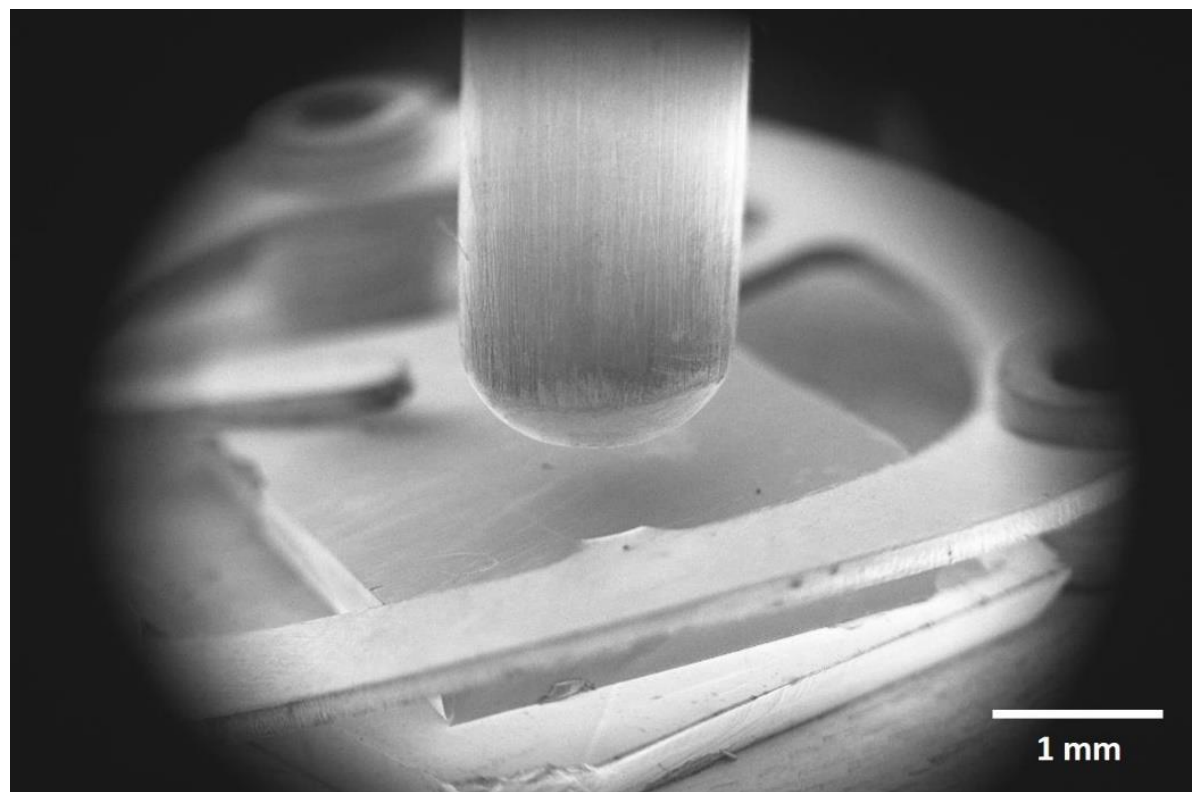

b)

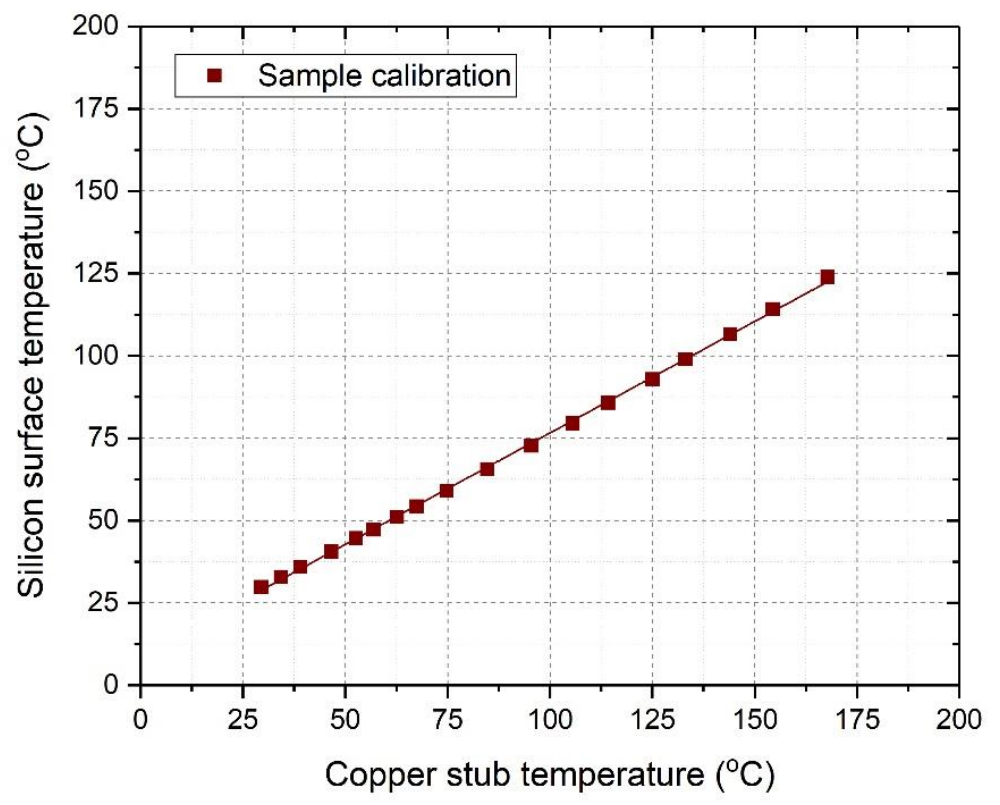

Figure S91 a) Silicon surface temperature was measured using the previously calibrated thermocouple, b) Sample side thermal calibration showing the correlation between the silicon surface temperature and the copper stub temperature. 
12. Locking of Glass Transition Temperatures through Photoisomerization

PSS 340: $83 \%$ Z

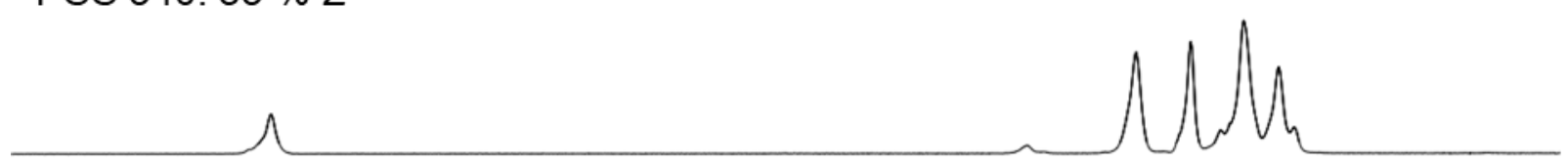

PSS 365: $57 \%$ Z

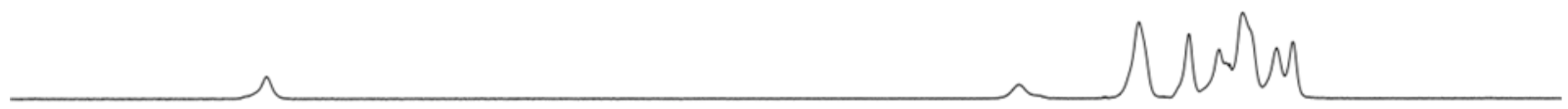

PSS 375: $38 \%$ Z

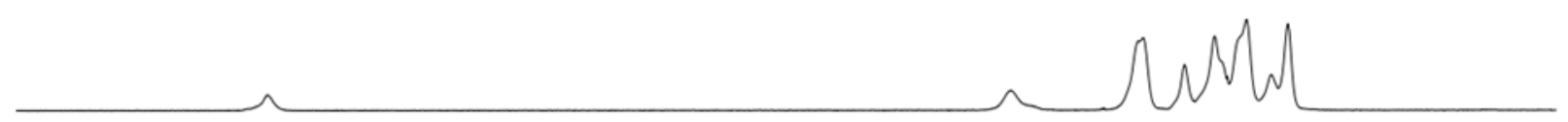

PSS 394: $10 \%$ Z
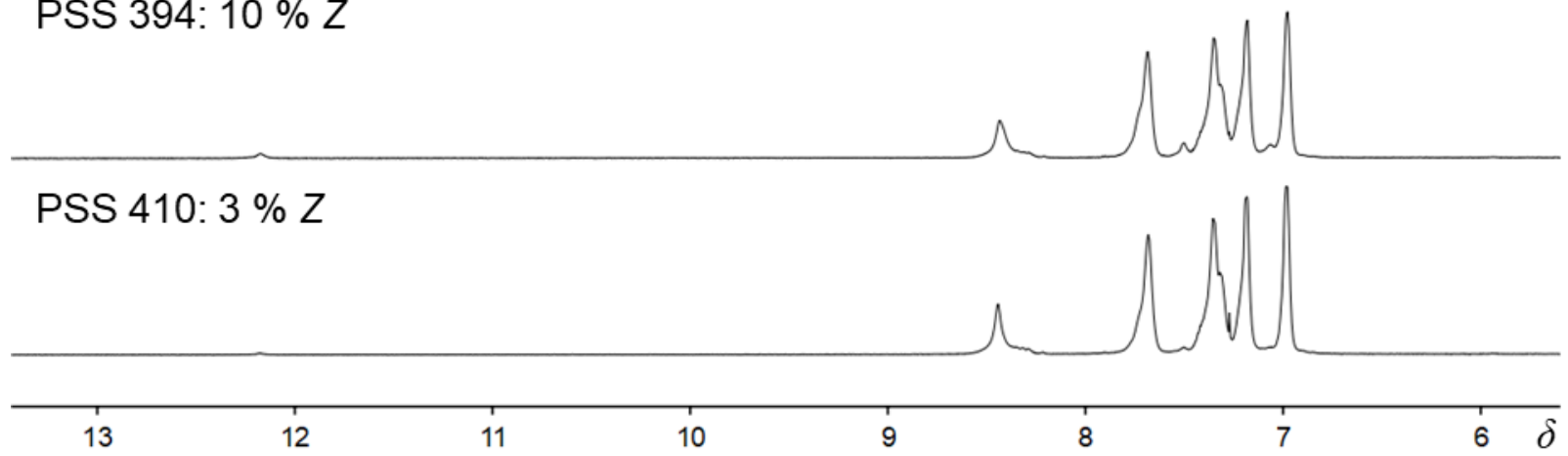

Figure S92. Different photostationary states of polymer P5 obtained using various irradiation wavelengths. 
a)

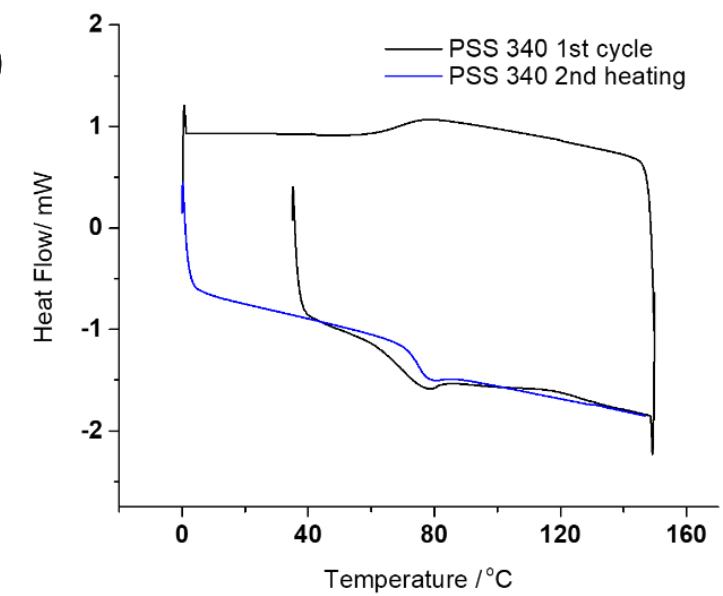

C)

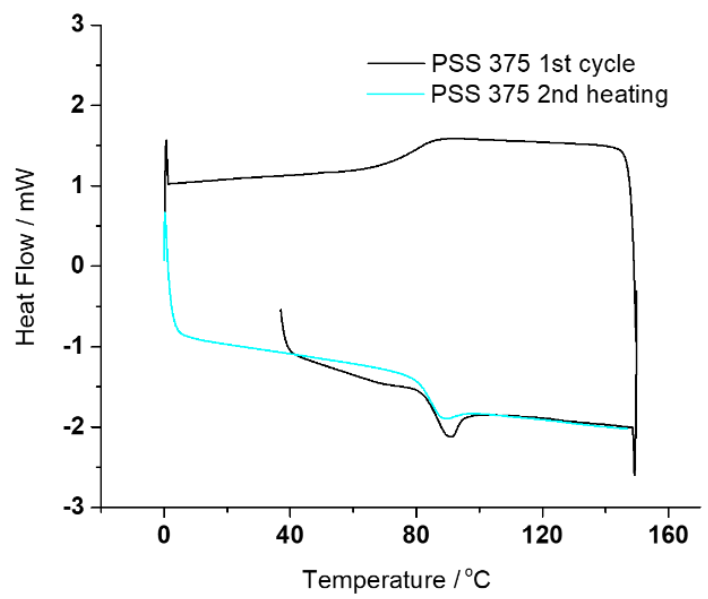

e)

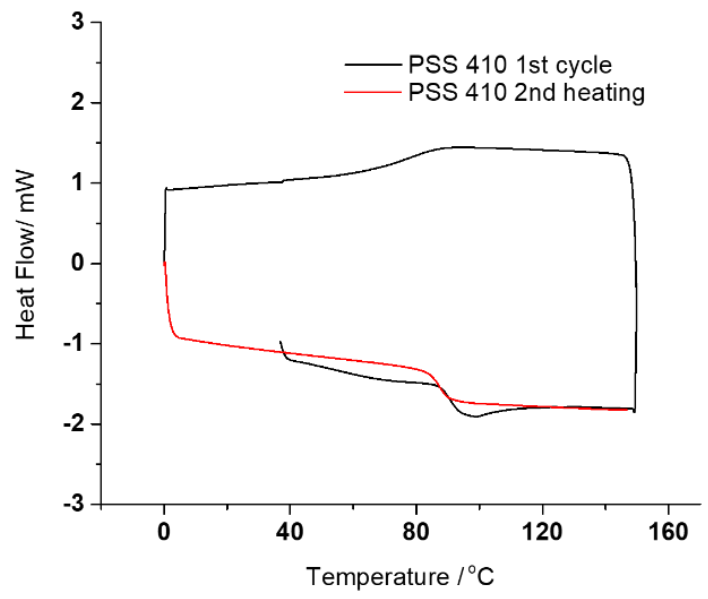

b)

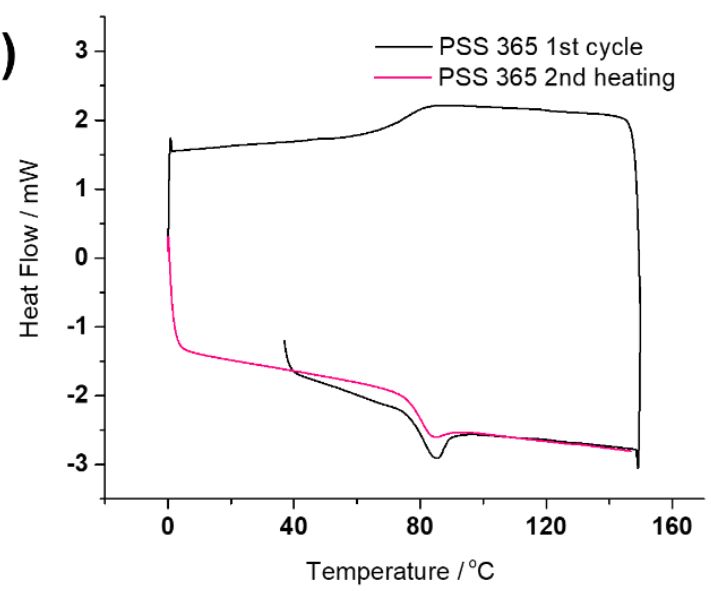

d)

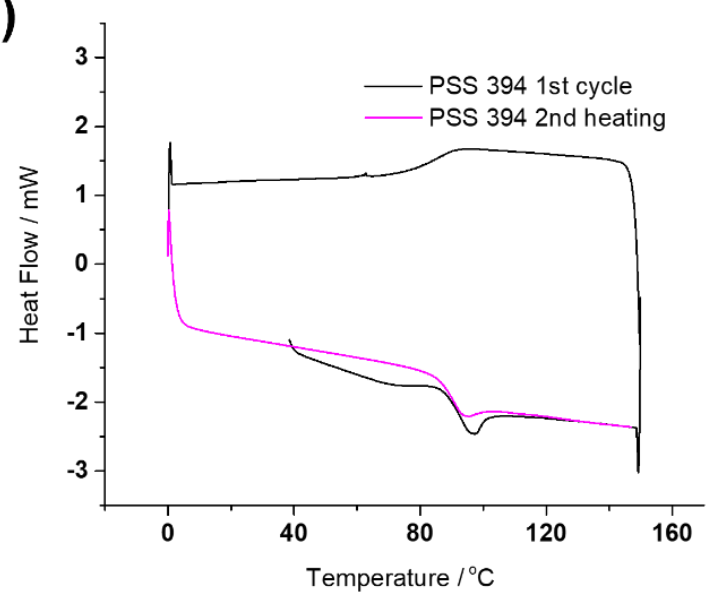

f)

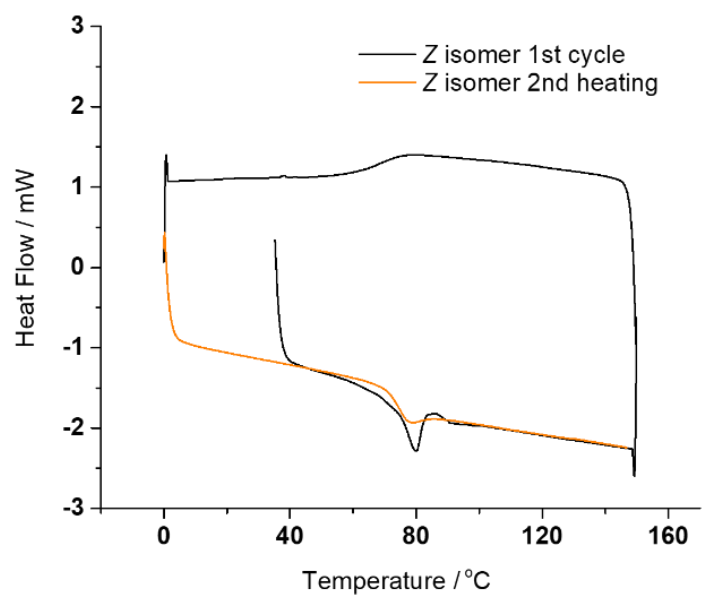

Figure S93. DSC curves of different PSSs of P5 after the first and second heating cycles at PSS a) 340 ; b) 365 ; c) 375 ; d) 394 ; e) 410 ; and of the f) $Z$ isomer 


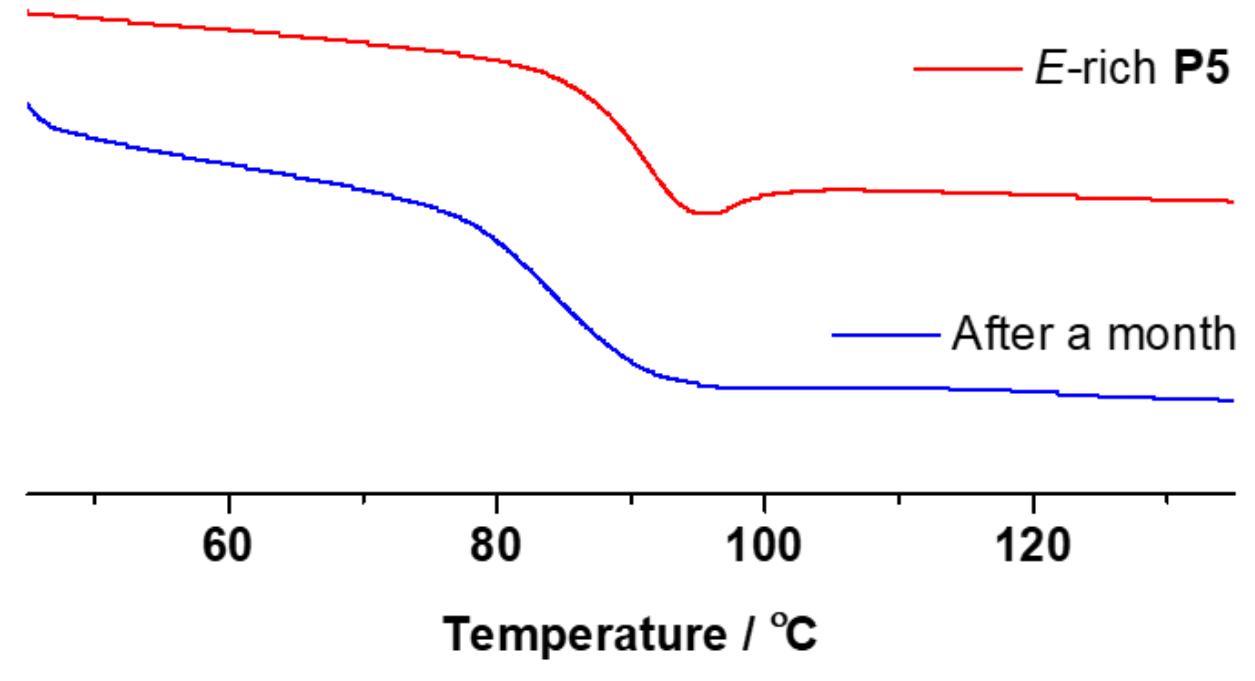

Figure S94. DSC curves of $E$-rich P5 taken at a month interval. The sample was stored at room temperature during this period of time. The second heating cycle is shown in both cases. 


\section{References}

S1 Li, Q., Qian, H., Shao, B., Hughes, R. P., Aprahamian, I. J. Am. Chem. Soc., 2018, 140, $11829-11835$.

S2 Ni, K., Meng, L.G., Ruan, H., Wang, L. Chem. Commun. 2019, 55, 8438-8441.

S3 Khan, S., Ahmed, Q. N. Eur. J. Org. Chem., 2016, 32, 5377-5385.

S4 M. J. Moran, Mitchell Magrini, David M. Walba, I. Aprahamian. J. Am. Chem. Soc. 2018, 140, $13623-13627$.

S5 W. C. Oliver, G. M. Pharr, J. Mater Res. 2004, 19, 3-20.

S6 Conte, M., Mohanty, G., Schwiedrzik, J. J., Wheeler, J. M., Bellaton, B., Michler, J., Randall, N. X. Rev. Sci. Instrum. 2019, 90, 45105.

S7 Wheeler, J. M., Michler, J. Rev. Sci. Instrum. 2013, 84, 45103. 